\title{
Search for resonances in the mass distribution of jet pairs with one or two jets identified as $b$-jets in proton-proton collisions at $\sqrt{s}=13 \mathrm{TeV}$ with the ATLAS detector
}

\author{
M. Aaboud et al. \\ (ATLAS Collaboration)
}

(Received 24 May 2018; published 30 August 2018)

\begin{abstract}
A search for new resonances decaying into jets containing $b$-hadrons in $p p$ collisions with the ATLAS detector at the LHC is presented in the dijet mass range from 0.57 to $7 \mathrm{TeV}$. The data set corresponds to an integrated luminosity of up to $36.1 \mathrm{fb}^{-1}$ collected in 2015 and 2016 at $\sqrt{s}=13 \mathrm{TeV}$. No evidence of a significant excess of events above the smooth background shape is found. Upper cross-section limits and lower limits on the corresponding signal mass parameters for several types of signal hypotheses are provided at 95\% C.L. In addition, 95\% C.L. upper limits are set on the cross sections for new processes that would produce Gaussian-shaped signals in the di- $b$-jet mass distributions.
\end{abstract}

DOI: 10.1103/PhysRevD.98.032016

\section{INTRODUCTION}

New heavy particles that couple to quarks or gluons are predicted by several extensions of the Standard Model (SM) [1-5]. There is a renewed interest as these new particles can act as mediators for dark matter (DM) interactions [6-10]. Such heavy particles can be produced in proton-proton collisions at relatively high rates thanks to their possibly strong coupling. The new particles can decay into quarks and gluons, that hadronize and form jets that are observable in the detector. Such a decay will produce dijet systems with an invariant mass around the mass of the new particle, appearing as an excess above the continuum background. This analysis searches for a resonant excess in the dijet mass distribution.

The dijet mass range explored in previous analyses depends on the available center-of-mass energy as well as on the size of the data sample. Past dijet searches have investigated the dijet mass ranges $110-350 \mathrm{GeV}$ at the $\mathrm{S} p \bar{p} S$ collider [11] and 260-1400 GeV [12], 250-1100 GeV [13] at the Tevatron. At the LHC, the most recent CMS search covers 0.6-7.5 TeV [14], while the last ATLAS search covers $0.45-6.5 \mathrm{TeV}[15,16]$.

Searches restricted to final states involving jets identified as containing a $b$-hadron have an increased sensitivity to certain scenarios, e.g., to particles that preferentially decay into $b \bar{b}$ quark pairs as predicted by some dark-matter

*Full author list given at the end of the article.

Published by the American Physical Society under the terms of the Creative Commons Attribution 4.0 International license. Further distribution of this work must maintain attribution to the author(s) and the published article's title, journal citation, and DOI. Funded by SCOAP. models $[17,18]$. But the sensitivity can be improved even for resonances without an enhanced $b \bar{b}$ decay mode, like many $Z^{\prime}$ models described below, if the search suffers from non- $q \bar{q}$ backgrounds, in particular gluon radiation. Such searches have been performed by CDF covering the mass range 250-750 GeV [19], by CMS covering $0.3-4 \mathrm{TeV}[20,21]$ and by ATLAS covering $1-5 \mathrm{TeV}$ [22]. So far no deviations from the Standard Model have been found.

Compared to previous collider searches that have explored the mass region below $1 \mathrm{TeV}$, the LHC can provide higher sensitivity and cover yet unexplored coupling values due to the increase in parton luminosity [23]. Consequently resonance searches in this mass range are still of interest. In particular, some dark-matter models predict such particles $[16,17]$. In this paper an extension of the ATLAS search into this lower-mass region is made possible by a new trigger strategy, identifying two $b$-jets at trigger level. This strategy is able to cope with the large event rate in the lower dijetmass region. The search presented in this paper probes the mass range $0.57-7 \mathrm{TeV}$.

The results are interpreted in the context of several benchmark models. An excited $b^{*}$-quark, with a dominant decay mode to $b g$, is used as the benchmark for events with at least one jet identified containing $b$-hadrons: the $\geq 1$ $b$-tag category. Excited quarks arise from compositeness models $[4,5]$. Models featuring an additional gauge boson called $Z^{\prime}$ [1-3], including a dark-matter model with a $Z^{\prime}$ mediator [6,7], are considered in the two $b$-tags category. The leading-order Feynman diagrams for these processes are shown in Fig. 1. Further details can be found in Sec. III. In addition, model-independent limits are set on generic resonance signals that have a Gaussian reconstructed 


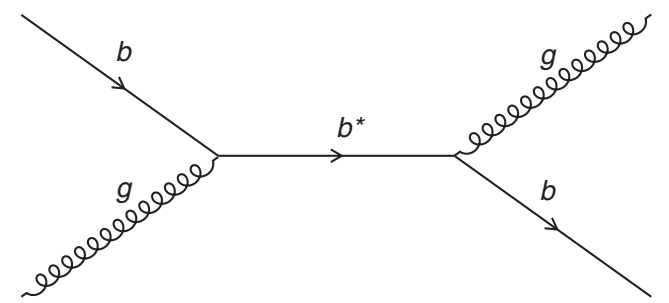

(a)

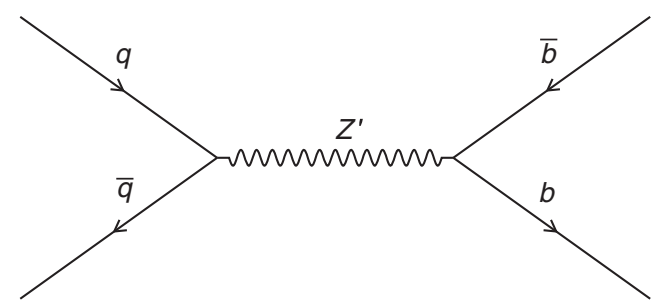

(b)

FIG. 1. Example of the leading-order Feynman diagram for production and decay of (a) $b^{*}$ and (b) $Z^{\prime}$ into final states involving $b$ quarks.

shape. These limits assume, after applying the selection, a narrow-resonance signal shape with an intrinsic width that can be safely truncated or neglected, so that the reconstructed mass distribution reflects the experimental resolution and can be approximated by a Gaussian distribution [24].

\section{ATLAS DETECTOR}

The ATLAS detector [25] at the LHC covers nearly the entire solid angle around the collision point. ${ }^{1}$ It consists of an inner tracking detector surrounded by a thin superconducting solenoid, electromagnetic and hadronic calorimeters, and a muon spectrometer incorporating three large superconducting toroid magnets. The inner-detector system (ID) is immersed in a $2 \mathrm{~T}$ axial magnetic field and provides charged-particle tracking in the range $|\eta|<2.5$.

A high-granularity silicon pixel detector covers the vertex region and typically provides three measurements per track. A new inner pixel layer, the insertable B-layer [26,27], was added during the 2013-2014 LHC shutdown. It is located at a mean sensor radius of $32 \mathrm{~mm}$ from the beam line, providing a fourth pixel hit. The pixel detector is followed by a silicon microstrip tracker, which usually provides four two-dimensional measurement points per track. These silicon detectors are complemented by a transition radiation tracker, which enables radially extended track reconstruction up to $|\eta|=2.0$. The transition radiation tracker also provides electron identification information based on the fraction of hits (typically 30 in total) that deposit energy above a threshold corresponding to transition radiation.

The calorimeter system covers the pseudorapidity range $|\eta|<4$.9. Within the region $|\eta|<3$.2, electromagnetic

\footnotetext{
${ }^{1}$ ATLAS uses a right-handed coordinate system with its origin at the nominal interaction point (IP) in the center of the detector and the $z$ axis along the beam pipe. The $x$ axis points from the IP to the center of the LHC ring, and the $y$ axis points upward. Cylindrical coordinates $(r, \phi)$ are used in the transverse plane, $\phi$ being the azimuthal angle around the $z$ axis. The pseudorapidity is defined in terms of the polar angle $\theta$ as $\eta=-\ln \tan (\theta / 2)$. Angular distance is measured in units of $\Delta R \equiv \sqrt{(\Delta \eta)^{2}+(\Delta \phi)^{2}}$.
}

calorimetry is provided by barrel and endcap highgranularity lead/liquid-argon (LAr) electromagnetic calorimeters, with an additional thin LAr presampler covering $|\eta|<1.8$, to correct for energy loss in material upstream of the calorimeters. Hadronic calorimetry is provided by the steel/scintillator-tile calorimeter, segmented into three barrel structures within $|\eta|<1.7$, and two copper/LAr hadronic endcap calorimeters. The solid angle coverage is completed with forward copper/LAr and tungsten/LAr calorimeter modules optimized for electromagnetic and hadronic measurements respectively.

A two-level trigger system is used to select interesting events. The first trigger level is implemented in hardware and uses a subset of detector information to reduce the event rate to a design value of at most $100 \mathrm{kHz}$. This is followed by a software-based high-level trigger (HLT) which reduces the event rate to about $1 \mathrm{kHz}$.

\section{SIMULATED SIGNAL SAMPLES}

The Monte Carlo (MC) simulation is used to generate samples describing the benchmark signal models under consideration. These signal samples were generated with Pythia8 [28] using the A14 set of tuned parameters [29] and the NNPDF2.3 PDF set [30]. The EvTGen decay package [31] is used for bottom and charm hadron decays. The generated samples were processed with the ATLAS detector simulation [32], which is based on the GEANT4 package [33]. To account for additional proton-proton interactions (pileup), further minimum-bias interactions were generated using PYTHIA8 and the MSTW2008LO PDF set [34] and superimposed on the hard-scattering events. The MC samples were reweighted to match the distribution of the number of collisions per bunch crossing observed in the data. For basic background validation a leading-order multijet sample was generated with PYTHIA8 and the same parameters and PDF set used for the signal models. The same reconstruction software was run on the simulated events as was used for recorded collision data.

Signal events in the excited $b^{*}$-quark model were generated with the compositeness scale $\Lambda$ set to the excited-quark mass $m_{b^{*}}$ and an intrinsic decay width of $\Gamma \sim 0.006 \times m_{b^{*}}$. The branching fraction for the dominant 
decay $b^{*} \rightarrow b g$ is $85 \%$. The remaining decay modes are $b^{*} \rightarrow b \gamma, b^{*} \rightarrow b Z^{0}$ and $b^{*} \rightarrow t W^{-}$. The leading-order (LO) cross section for a $2.5 \mathrm{TeV} b^{*}$-quark is $123 \mathrm{fb}$ [28].

Three models with a $Z^{\prime}$ gauge boson are considered. In the sequential standard model (SSM), the $Z^{\prime}$ boson has the same couplings to SM fermions as the SM $Z$ boson and the bottom-quark decay branching fraction $\mathcal{B}\left(Z^{\prime} \rightarrow b \bar{b}\right)$ is $13.8 \%$. The leptophobic $Z^{\prime}$ model differs by having vanishing couplings to leptons. The corresponding value of $\mathcal{B}\left(Z^{\prime} \rightarrow b \bar{b}\right)$ is $18.9 \%$ in the mass ranges considered. The intrinsic width of the $Z^{\prime}$ bosons are set to $3 \%$ of the resonance mass [1]. The leading-order PYTHIA8 SSM and leptophobic $Z^{\prime}$ cross sections were corrected to next-toleading order (NLO) using cross sections calculated at LO and at NLO using MADGRAPH5_AMC@NLO [35], with the NNPDF2.3 LO and NLO PDF sets, respectively. The NLO prediction uses a model of neutral vector bosons implemented in FeynRules [36] with NLO terms evaluated via NLOCT package [37]. The NLO cross section times branching fraction $\sigma \cdot \mathcal{B}\left(Z^{\prime} \rightarrow b \bar{b}\right)$ for a $2 \mathrm{TeV}$ SSM neutral vector boson is $0.10 \mathrm{fb}[28,35]$. For both models, only decays into $b$-quark pairs were simulated.

Lastly, a simplified dark-matter model [9] with a $Z^{\prime}$ axial-vector mediator is considered. The mediator to SM quark coupling $\left(g_{\mathrm{SM}}\right)$ was set to 0.1 or 0.25 , the mediator to axial DM coupling to 1.0 and the mass of the darkmatter particle was fixed to $10 \mathrm{TeV}$ within the scope of the Ref. [9]. The intrinsic width was calculated by MADGRAPH5_AMC@NLO [35]. The LO cross section times branching fraction $\sigma \cdot \mathcal{B}\left(Z^{\prime} \rightarrow q \bar{q}\right)$ for a $1 \mathrm{TeV}$ axial-vector mediator with $g_{\mathrm{SM}}=0.1$ is $2.7 \mathrm{fb}$ [28].

\section{DATA SAMPLES AND EVENT SELECTION}

The data for this analysis were collected by the ATLAS detector in $p p$ collisions with a center-of-mass energy of $\sqrt{s}=13 \mathrm{TeV}$. The data set for the high dijet-mass region $m_{j j}>1.2 \mathrm{TeV}$ was recorded by selecting events from an inclusive jet trigger requiring at least one jet with a transverse momentum $p_{\mathrm{T}}$ above $380 \mathrm{GeV}$, and corresponds to an integrated luminosity of $3.2 \mathrm{fb}^{-1}$ in 2015 and $32.9 \mathrm{fb}^{-1}$ in 2016. Events for the low dijet-mass region $570 \mathrm{GeV}<m_{j j}<1.5 \mathrm{TeV}$ were recorded using a dijet trigger employing an online algorithm to identify two jets containing $b$-hadrons and having transverse momentum $p_{\mathrm{T}}$ above 150 and $50 \mathrm{GeV}$, respectively. The above transverse momentum requirements are fully efficient in the quoted mass range. This trigger overcomes the limitation related to the high inclusive single jet trigger rate. Because the $b$-jet trigger was active only for parts of the data taking period, the total integrated luminosity that the low dijet-mass sample corresponds to is $24.3 \mathrm{fb}^{-1}$ in 2016. The $b$-jet trigger chain [38] starts by requiring an energy deposit measured with coarse granularity $(\Delta \phi \times \Delta \eta=0.2 \times 0.2)$ in the calorimeter at the first trigger level. In the HLT, a two-step tracking algorithm is run. First, a fast tracking stage is used to find the primary vertex of the event. The results from this first stage seed precision tracking. The output of this tracking stage provides the input for the $b$-jet identification algorithms, which are based on the offline tools described further below. The identification efficiency is $60 \%$ per $b$-jet at trigger level when integrated over transverse momentum $p_{\mathrm{T}}$ and pseudorapidity $\eta$.

Offline jets are reconstructed from topological clusters of energy deposits in the calorimeters [39] with the anti- $k_{t}$ algorithm $[40,41]$ with a radius parameter of 0.4 . Jet energies and directions are corrected by the jet calibrations as described in Ref. [42]. Jets containing a $b$-hadron are identified using a multivariate algorithm $[43,44]$. This algorithm makes use of the impact parameters of tracks and the reconstructed displaced vertices in the ID. The offline $b$-tagging efficiency operating points are determined on a $t \bar{t}$ sample when integrated over $p_{\mathrm{T}}$ and $\eta$ [45]. In the high-mass region, an $85 \%$ efficiency offline $b$-tagging operating point is employed. In the low-mass region, a $70 \%$ offline efficiency $b$-tagging operating point is adopted in addition to the online $b$-tagging requirement, because the online $b$-identification is only partially correlated to the offline $b$-tagging. The online $b$-tagging algorithm is not fully emulated in MC and the tagging efficiency is needed to estimate the signal acceptance. The online $b$-tagging efficiency is measured using a high $b$-jet purity dilepton $t \bar{t}$ sample. The offline $b$-tagging operating points have been optimized in order to maximize the overall sensitivity.

In order to ensure full trigger efficiency and lower pileup contamination, the event selection requires a minimum transverse momentum of $p_{\mathrm{T}}>430 \mathrm{GeV}$ and $p_{\mathrm{T}}>80 \mathrm{GeV}$ for the leading and subleading jet, respectively. The requirement on the leading jet is relaxed to $200 \mathrm{GeV}$ for the low-mass region, corresponding to the reduced transverse momentum requirement in the trigger. Both jets are required to have pseudorapidity $|\eta|<2.0$ to allow fully efficient $b$-jet identification in the two mass regions. To reduce background from multijet production and enhance s-channel signal processes, the rapidity difference $y^{*}=\left(y_{1}-y_{2}\right) / 2$ between the two leading jets is required to be $\left|y^{*}\right|<0.8$. In the low-mass region this requirement is tightened to $\left|y^{*}\right|<0.6$ to avoid regions of reduced trigger efficiency at the lower mass boundary.

In the analysis, one or both of the leading jets are required to be identified as $b$-jets. The per-event efficiencies, taking the $b$-tagging requirement(s) into account, are shown as functions of the reconstructed invariant mass of the two leading jets, $m_{j j}$, for several signal models in Fig. 2. Events from the $Z^{\prime}$ model have a higher event-tagging efficiency than for $b^{*}$ events in the inclusive " $1 \mathrm{~b}$ " category because $Z^{\prime}$ events contain two $b$-quarks in the final state. In the high-mass region, the $b^{*} \rightarrow b g$ decay can be followed by the gluon splitting into a $b \bar{b}$ pair, which 


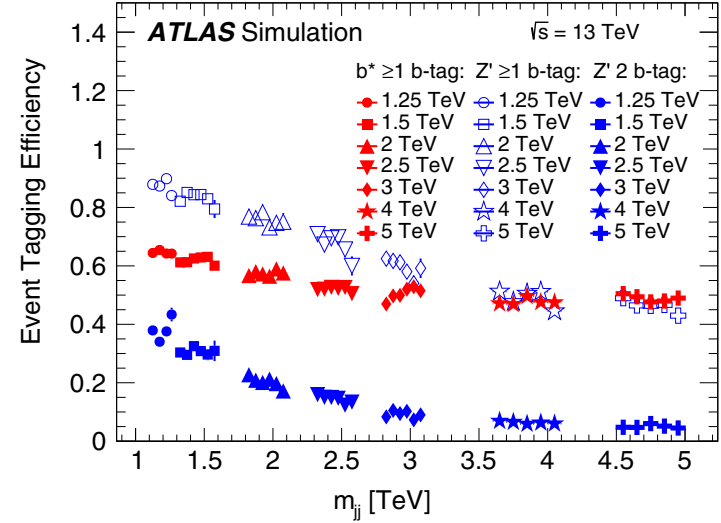

(a) High-mass region

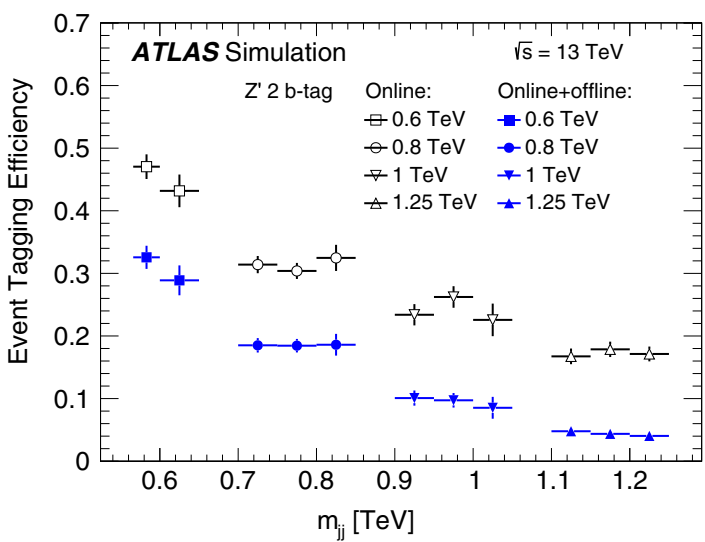

(b) Low-mass region

FIG. 2. The per-event $b$-tagging efficiencies after the event selection, as a function of the reconstructed invariant mass, $m_{j j}$. Events are classified into single $b$-tagged " $1 \mathrm{~b}$ " or double $b$-tagged " $2 \mathrm{~b}$ " categories. The efficiencies are shown for simulated event samples corresponding to (a) seven different $b^{*}$ and $Z^{\prime}$ resonance masses in the high-mass region and (b) four different $Z^{\prime}$ resonance masses in the low-mass region. For each generated resonance mass, several reconstructed mass points around the resonance mass are shown. (b) Efficiencies of identifying an event with two $b$-jets at trigger level only (online) and when requiring offline confirmation (online + offline) are shown.

therefore enhances the event $b$-tagging efficiency for $b^{*}$ events relative to the $Z^{\prime}$ signal.

\section{ANALYSIS}

The observed dijet mass distribution of the two leading jets in the high-mass event selection $\left(m_{j j}>1.2 \mathrm{TeV}\right)$, where at least one ( $\geq 1 b$-tag) or both ( $2 b$-tags) jets are identified as $b$-jets, is inspected for resonant contributions from new-physics scenarios. In the low-mass analysis $\left(570 \mathrm{GeV}<m_{j j}<1.5 \mathrm{TeV}\right)$ only a selection with two $b$-tags is considered due to the trigger selection. The treatment of the 2 - $b$-tags overlap region $\left(1.2 \mathrm{TeV}<m_{j j}<\right.$ 1.5 TeV) is discussed in Sec. VII.

The dominant background arises from multijet final states. While the shape of the $m_{j j}$ distribution in data is found to be in good agreement with the PYTHIA8 multijet MC simulation, the normalization is not. In this analysis the background is evaluated from a fit to the mass distribution in data.

Previous dijet resonance searches $[15,46]$ have found that the following fit function:

$$
f(x)=p_{1}(1-x)^{p_{2}}(x)^{p_{3}+p_{4} \ln x+p_{5}(\ln x)^{2}},
$$

where $p_{i}$ are fit parameters and $x \equiv m_{j j} / \sqrt{s}$, provides a good global fit to dijet mass distributions in data as well as leading-order and next-to-leading-order simulations of QCD dijet production, where $p_{5} \equiv p_{4} \equiv 0$ [46] or $p_{5} \equiv 0$ [15]. However, it is found that Eq. (1) no longer provides an adequate description of the data for the whole mass distribution comprising the high-mass and low-mass regions. This effect is attributed to a larger data sample than in previous analyses that employed the global fit strategy, in conjunction with the shaping of the $b$-tagged dijet mass distribution due to the $p_{\mathrm{T}}$ dependence of the $b$-tagging efficiency and variations of the quark flavor fractions as a function of $p_{\mathrm{T}}$. The background estimate is therefore derived from a sliding-window fit by using the fit function from Eq. (1) with four or five fit parameters, and by fitting only restricted regions of the spectrum at a time. This technique was introduced in the most recent ATLAS dijet resonance search [15] and is briefly described here. The number of fit parameters of the sliding-window fit are chosen to have the largest possible window size for a fit function with the fewest number of parameters. The four-parameter fit [where $p_{5}$ is set to zero in Eq. (1)] is chosen for the high-mass $2-b$-tags selection, while the fiveparameter fit is chosen for the low-mass and the inclusive $\geq 1 b$-tag selections. For the low-mass selection the window size is chosen to comprise 14 out of 31 total bins, whereas for the high-mass selection the window size corresponds to 16 bins for the 2 - $b$-tags selection and 22 bins for the $\geq 1$ $b$-tag selection out of a total number of 75 bins. The bin width follows approximately the $m_{j j}$ invariant mass resolution as derived from the MC simulation of multijet processes. The bin width increases from about $20 \mathrm{GeV}$ at a mass of $500 \mathrm{GeV}$ to about $130 \mathrm{GeV}$ at a mass of $7 \mathrm{TeV}$. The background prediction over the full mass range is constructed in each mass bin by evaluating the fit function in the window centered around that bin. At the low and high edge of the mass distribution, the sliding-window regions do not extend outside the considered mass range.

The validity of this background-fitting method is tested in data control regions, where no offline $b$-jet identification is required and the MC-estimated $b$-tagging efficiencies are applied as a weight. Representative background data sets are created by injecting Poisson fluctuations into the data 
control regions. Spurious-signal tests are performed to verify that no artifact is created during the fitting procedure by fitting hundreds of representative background data sets, and then checking the flatness of the probability returned by the BumpHunter algorithm [47] as detailed below. The fit is shown to be robust against spurious signals. In addition, signal injection tests are performed and good linearity between the injected and extracted signal is observed for the full range of signal widths considered. No sensitivity reduction due to the choice of window size is found.

For both the low-mass and high-mass $2-b$-tag selections the background prediction covers the full $m_{j j}$ mass region, where the lower boundaries are defined by the plateau

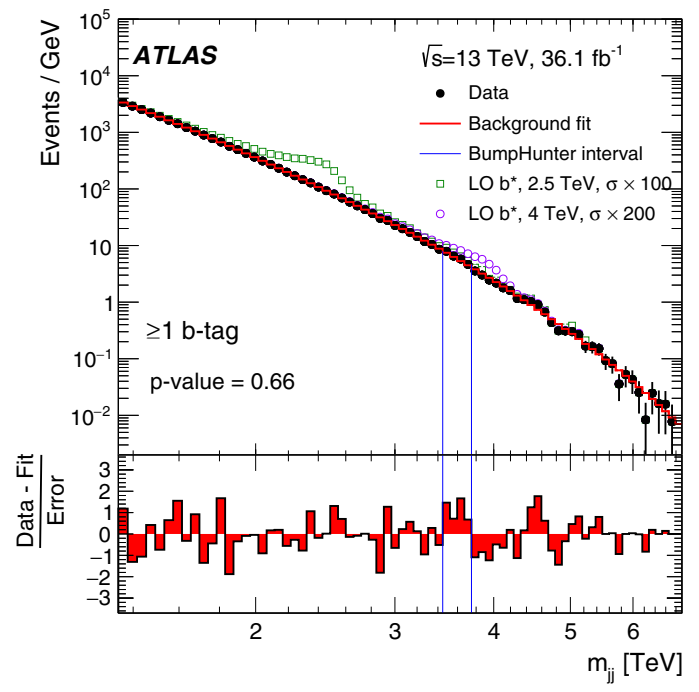

(a) Inclusive one $b$-tag, high mass

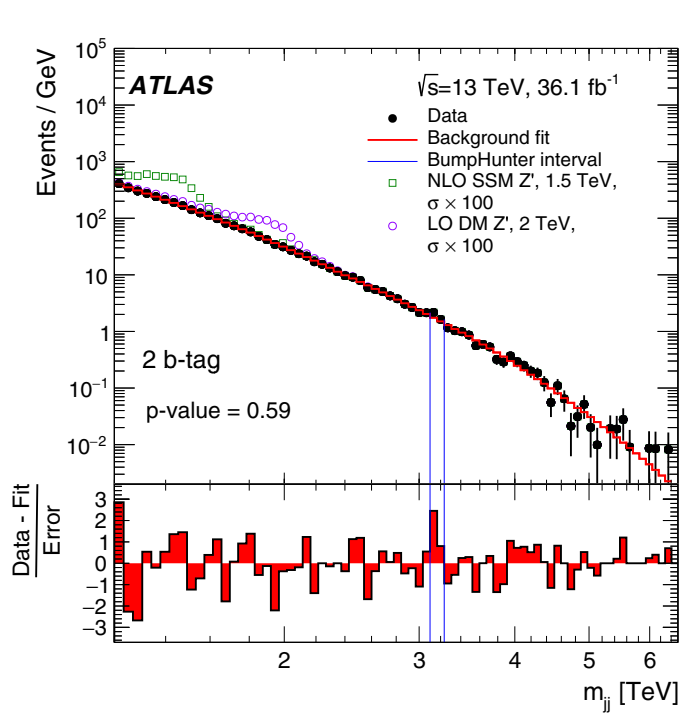

(b) Two $b$-tag, high mass region of the trigger as defined in Sec. IV. For the highmass inclusive $\geq 1 b$-tag selection, studies of the validity of the fit required an increase of the lower mass boundary from 1.2 to $1.3 \mathrm{TeV}$. The largest value of $m_{j j}$ is measured to be $6.77 \mathrm{TeV}$ with one $b$-tag and $6.31 \mathrm{TeV}$ with two $b$-tags.

Figure 3 shows the $m_{j j}$ distributions, overlaid with the fit results and examples of the potential signals described in Sec. III. The lower panel in each plot of Fig. 3 shows the significance of the bin-by-bin differences between the data and the fit, as calculated from Poisson probabilities, considering only statistical uncertainties. The BumpHunter algorithm is used to evaluate the statistical significance of any localized excess in the dijet mass distributions in data relative to the fitted background estimate. The algorithm

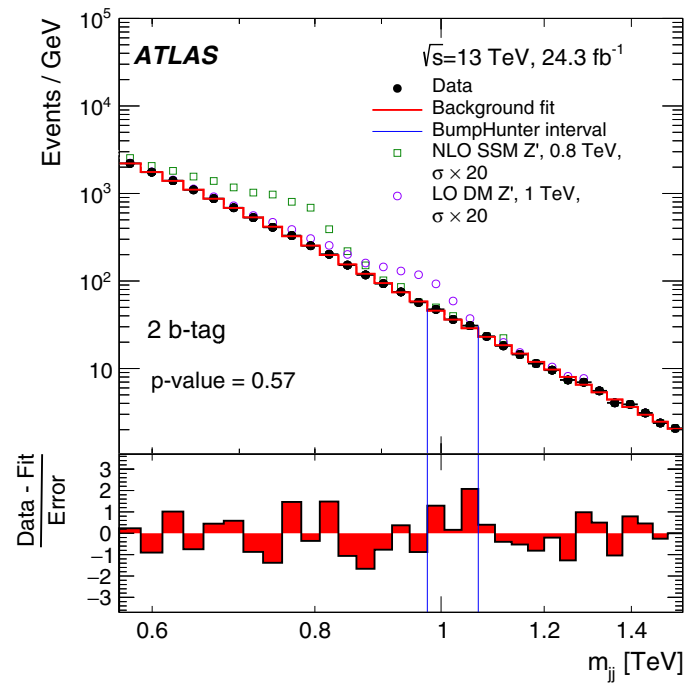

(c) Two $b$-tag, low mass

FIG. 3. Dijet mass spectra after the background only fit with the background prediction together with the result from the BumpHunter (see text for details). The plots show (a) the inclusive 1- $b$-tag high-mass region, (b) the high-mass region with two $b$-tags, and (c) the low-mass region with two $b$-tags. The potential signals are overlaid on top of the data. 
calculates the significance of any excess found in contiguous mass intervals in all possible locations of the binned $m_{j j}$ distribution, between a width of two bins and a width of half of the distribution. The intervals $3448-3749 \mathrm{GeV}$, 3100-3235 GeV, and 976-1068 GeV, indicated by two vertical lines in each of the Figs. 3(a)-3(c), are identified as the most discrepant intervals in the inclusive 1-b-tag, the 2 - $b$-tags high-mass, and the 2- $b$-tags low-mass region, respectively. The purely statistical significance of each excess is evaluated using the ensemble of possible outcomes across all scanned intervals, by applying the algorithm to many pseudo-data samples drawn randomly from the background fit. The probability that statistical fluctuations of the background would produce an excess at least as significant as the one observed in the data, anywhere in the distribution, is $0.66,0.59$, and 0.57 for the inclusive 1-b-tag, the 2-b-tags high-mass, and the 2-b-tags low-mass region, respectively. Thus there is no evidence of a significant localized excess over the background estimate.

\section{SYSTEMATIC UNCERTAINTIES}

The systematic uncertainty of the background is estimated from the uncertainty associated with the choice of the fit function and the uncertainties in the values of the fit parameters. The uncertainty due to the choice of the fit function is determined by repeating the fit procedure with one additional parameter. For the four-parameter fit of the high-mass 2 - $b$-tags selection, $p_{5}$ is added as an additional free parameter, and for the five-parameter fit of the lowmass and inclusive $\geq 1 b$-tag selections a new parameter $p_{6}$ is introduced in Eq. (1) by redefining $x$ as $x \equiv m_{j j} / p_{6}$. The uncertainty is given by the average difference between the two fit results across a set of pseudo-data drawn via Poisson fluctuations from the nominal background prediction. The uncertainty due to the values of the fit parameters is taken to be the bin-by-bin root-mean-square of the fit results for all the pseudo-experiments using the nominal fit function.

The uncertainty in the MC-based signal expectation is dominated by the uncertainty in the modeling of the $b$-tagging efficiency $[43,45]$. This uncertainty grows with jet $p_{\mathrm{T}}$, with a smallest uncertainty of $2 \%$ for jets with $p_{\mathrm{T}}$ around $90 \mathrm{GeV}$ and up to $15 \%$ for jet $p_{\mathrm{T}}$ around $1.5 \mathrm{TeV}$. The $b$-jet calibration is based on identifying a high-purity sample of $b$-jets by selecting $t \bar{t}$ events [45]. The uncertainties are measured using data for jet $p_{\mathrm{T}}<300 \mathrm{GeV}$ and are extrapolated to jet $p_{\mathrm{T}}>300 \mathrm{GeV}$ by means of $\mathrm{MC}$ simulation by varying quantities in the simulation that are known to affect the $b$-tagging performance, such as the track impact-parameter resolution, the fraction of poorly measured tracks, the description of the detector material, and the track multiplicity per jet. The uncertainty in the impact-parameter resolution includes alignment effects, dead modules and additional material not properly modeled in the simulation, and is the dominant source of uncertainty for the $b$-tagging efficiency at high $p_{\mathrm{T}}$.

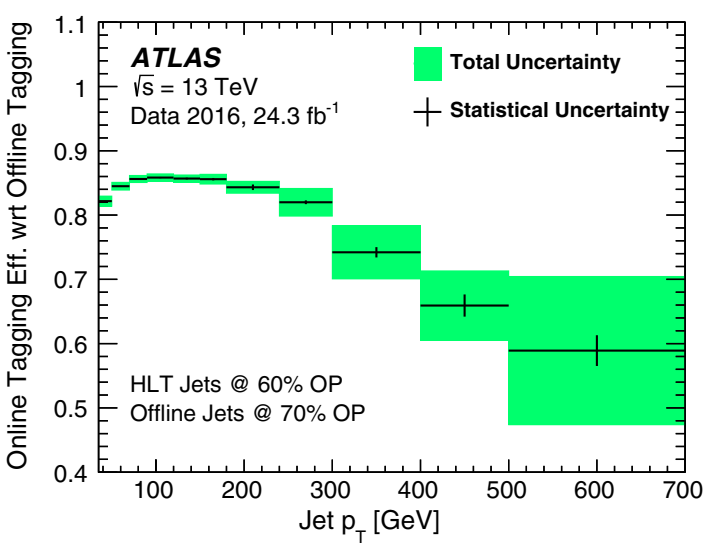

FIG. 4. The online $b$-tagging efficiency with respect to the offline $b$-tagging efficiency as a function of $p_{\mathrm{T}}$. The $b$-tagging online and offline working points correspond to an efficiency of $60 \%$ and $70 \%$, respectively.

Because the data set for the low-mass analysis is recorded using the $b$-jet trigger as described in Sec. IV, there is an additional systematic uncertainty associated with the $b$-jet trigger efficiency. It is extracted by comparing the $b$-jet trigger efficiency in 2016 data and MC simulation in a high-purity sample of $b$-jets selected from a dilepton $t \bar{t}$ sample by using similar procedures to those used to measure the offline $b$-tagging efficiencies. Uncertainties due to the mismodeling of the $b$-jet purity in simulation, mismodeling of the $b$-jet trigger efficiency for non $b$-jets, simulation statistical uncertainty, data statistical uncertainty (jet $p_{\mathrm{T}}<240 \mathrm{GeV}$ ) and simulation-based extrapolation (jet $p_{\mathrm{T}}>240 \mathrm{GeV}$ ) are taken into account. The per-jet uncertainty is estimated to be $1 \%-20 \%$ for jets with $p_{\mathrm{T}}$ of $35-700 \mathrm{GeV}$ (Fig. 4). The total uncertainty of the di- $b$-jet trigger efficiency comes from the per-jet $b$-tagging efficiency with an additional per-event uncertainty of $2 \%$ that covers differences in the primary vertex reconstruction.

The combined uncertainty in the jet energy scale (JES) and resolution (JER) is estimated using untagged jets in $13 \mathrm{TeV}$ data and simulation by following the methods described in Ref. [48]. The total uncertainty is found to be less than $2 \%$ of the jet $p_{\mathrm{T}}$ across the investigated mass range.

For $b$-tagged jets an additional uncertainty is assigned to the energy scale (bJES). It is estimated using MC samples and verified with data following the method described in Ref. [49]. Firstly, the ratio of the sum of track transverse momenta inside the jet to the total jet transverse momentum measured in the calorimeter is formed, and then this ratio is compared between data and simulation. This double ratio is then compared for inclusive jets and $b$-jets. The relative uncertainty is found to be at most $2.6 \%$ in the jet $p_{\mathrm{T}}$ spectrum of interest and is applied in addition to the nominal jet energy scale uncertainty.

The uncertainties described above are summarized in Table I. Other uncertainties that affect only the signal normalization, including the acceptance uncertainties 
TABLE I. Main sources of experimental systematic uncertainty in the low-mass and high-mass region. The background uncertainties quoted here are the uncertainties on the fitted background yield in each mass bin. The JES, JER, and bJES uncertainties quoted here are the uncertainties on the jet $p_{\mathrm{T}}$. The $b$-jet trigger and $b$-tagging uncertainties quoted here are the uncertainties on the per jet tagging efficiency.

\begin{tabular}{|c|c|c|c|}
\hline & \multirow[b]{2}{*}{ Low-mass region } & \multicolumn{2}{|c|}{ High-mass region } \\
\hline & & $\geq 1 b$ & $2 b$ \\
\hline \multicolumn{4}{|c|}{ Background systematic uncertainty } \\
\hline Fit function & $(0.01-1.2) \%$ & $(0.01-9) \%$ & $(0.01-10) \%$ \\
\hline Fit parameters & $(0.2-2.7) \%$ & $(0.3-34) \%$ & $(0.1-32) \%$ \\
\hline \multicolumn{4}{|c|}{ Signal systematic uncertainty } \\
\hline JES & $(1-1.2) \%$ & \multicolumn{2}{|c|}{$(1.2-1.6) \%$} \\
\hline JER & $(0.3-1) \%$ & \multicolumn{2}{|c|}{$(0.3-0.4) \%$} \\
\hline bJES & $2.6 \%$ & \multicolumn{2}{|c|}{$2.6 \%$} \\
\hline$b$-jet trigger & $(1-20) \%$ & \multicolumn{2}{|c|}{$\ldots$} \\
\hline$b$-tagging & $(7-20) \%$ & $(3-5) \%$ & $(10-30) \%$ \\
\hline
\end{tabular}

associated with the choice of PDF and the uncertainty in the integrated luminosity, are found to be negligible.

\section{INTERPRETATION}

Since no significant deviation from the expected background is observed, limits are set on processes that would lead to resonances in the considered mass distributions. The Bayesian method [50] is used to set $95 \%$ credibility-level (C.L.) upper limits on the cross section, where the $95 \%$ quantile of the posterior is taken as the upper limit. A Gaussian prior is used for each nuisance parameter corresponding to a systematic uncertainty, and a flat prior is used for the signal normalization. The expected limits as well as the $1 \sigma$ and $2 \sigma$ bands are calculated using

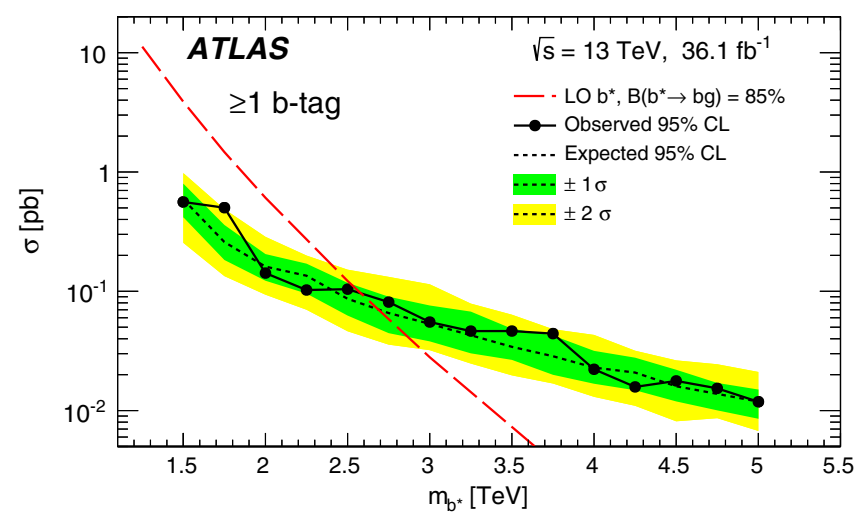

FIG. 5. Observed (filled circles) and expected (dotted line) $95 \%$ credibility-level upper limits on the cross section for the $b^{*}$ model. The dashed lines show the predicted LO cross section as defined in Sec. III. The plot shows the results in the high-mass region with inclusive $b$-jet selection.

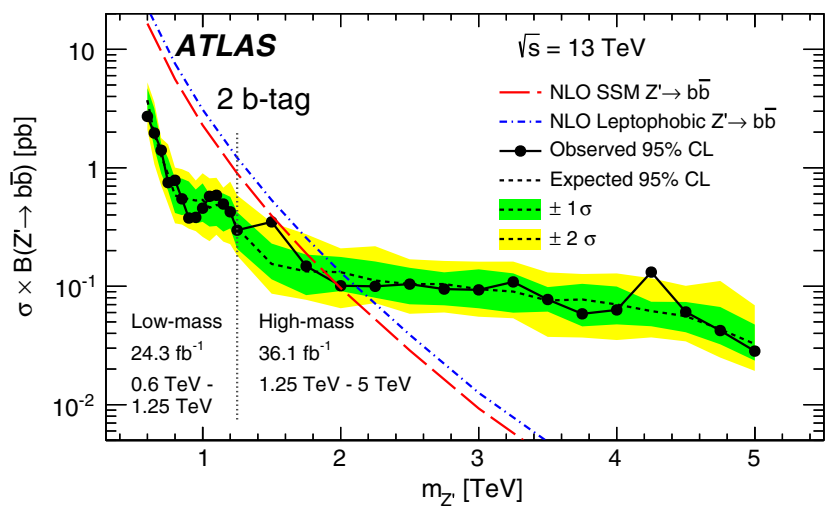

FIG. 6. Observed (filled circles) and expected (dotted line) $95 \%$ credibility-level upper limits on the cross section times branching ratio for the SSM and leptophobic $Z^{\prime}$ models. The dashed lines show the predicted NLO cross sections as defined in Sec. III. The plot shows the combined results in the low- and high-mass region (separated by the vertical dotted line) with two $b$-tags selection.

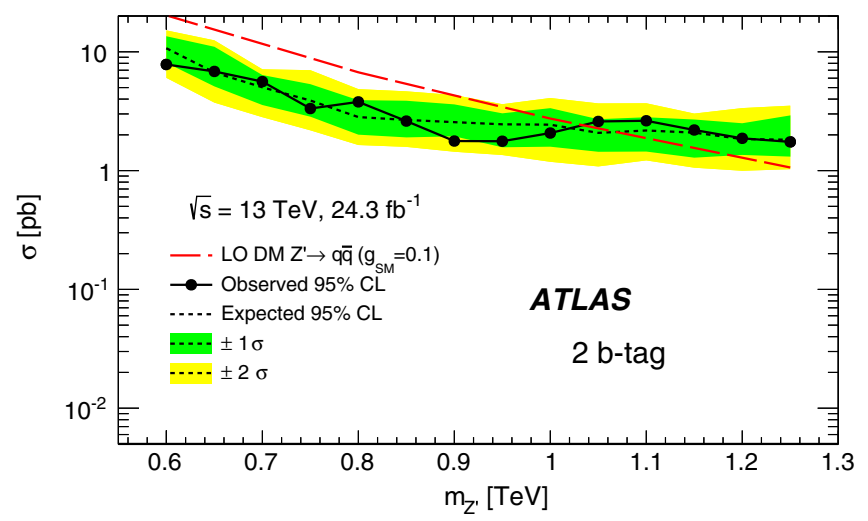

(a) Low-mass two $b$-tags selection

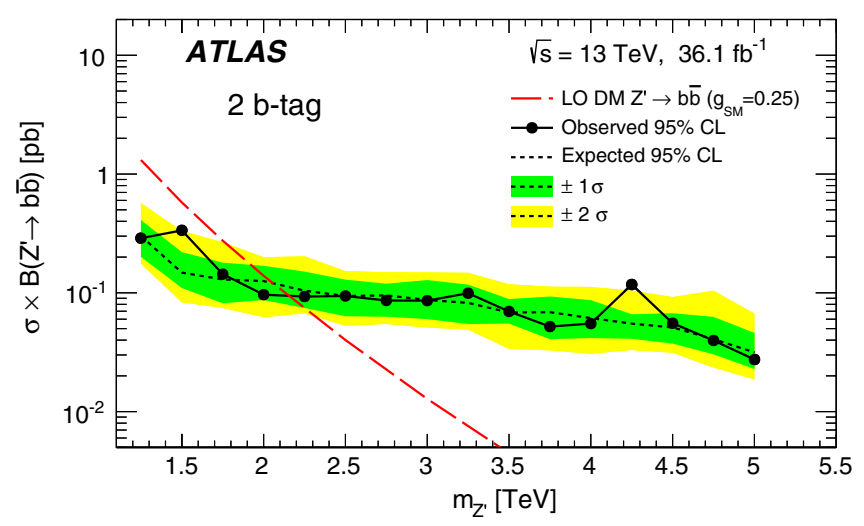

(b) High-mass two $b$-tags selection

FIG. 7. Observed (filled circles) and expected (dotted line) $95 \%$ credibility-level upper limits on the cross section for two different DM $Z^{\prime}$ models. In the low-mass region the $Z^{\prime}$ is expected to decay to all five quark flavors other than the top quark and the mediator to SM quark coupling $\left(g_{\mathrm{SM}}\right)$ equal to 0.1 is assumed, whereas in the high-mass selection only the decays $Z^{\prime} \rightarrow b \bar{b}$ are assumed with $g_{\mathrm{SM}}=0.25$. The dashed lines show the predicted LO cross sections as defined in Sec. III. 
pseudo-experiments generated from the background model by incorporating all systematic uncertainties in both the signal and background predictions. Template morphing [51] is utilized to interpolate between the resonance mass values of the signal hypotheses that are realized in $\mathrm{MC}$ simulation. The simulated models are described in detail in Sec. III. Theoretical uncertainties affecting the signal cross section are not considered. Figs. 5-7 show the cross-section limits for the $b^{*}$ signal using the inclusive $b$-jet selection, the $Z^{\prime}$ signal using the combined low- and high-mass $2-b$ tags selection, and the DM $Z^{\prime}$ signal in the low- and highmass 2 - $b$-tags region, respectively. The low- and high-mass selections overlap in the mass region between 1.2 and 1.5 TeV. For the combination the result with the better expected limit is chosen within the overlap region.

The cross-section limits are translated to limits on the following signal mass parameters. The $b^{*}$ model with an assumed branching fraction for $b^{*} \rightarrow b g$ of $85 \%$ is excluded at $95 \%$ C.L. for masses up to $2.6 \mathrm{TeV}$ using the inclusive single $b$-jet channel. The double $b$-jet channel is used to set limits at $95 \%$ C.L. which exclude masses up to 2.0 TeV for the SSM $Z^{\prime} \rightarrow b \bar{b}$ model and which exclude masses up to $2.1 \mathrm{TeV}$ for the leptophobic $Z^{\prime} \rightarrow b \bar{b}$ model with SM-value couplings to quarks. Mass limits on a darkmatter $Z^{\prime}$ depend on the decay mode and the coupling strength to quarks, $g_{\mathrm{SM}}$. Assuming only the decay $Z^{\prime} \rightarrow b \bar{b}$ and $g_{\mathrm{SM}}=0.25$, masses up to $2.1 \mathrm{TeV}$ are excluded at 95\% C.L. Assuming $Z^{\prime}$ decays to all five quark flavors other than the top quark and $g_{\mathrm{SM}}=0.1$, masses up to $1.03 \mathrm{TeV}$ are excluded at 95\% C.L.

In order to allow for limit setting on new-physics models beyond those considered in the current studies, limits are

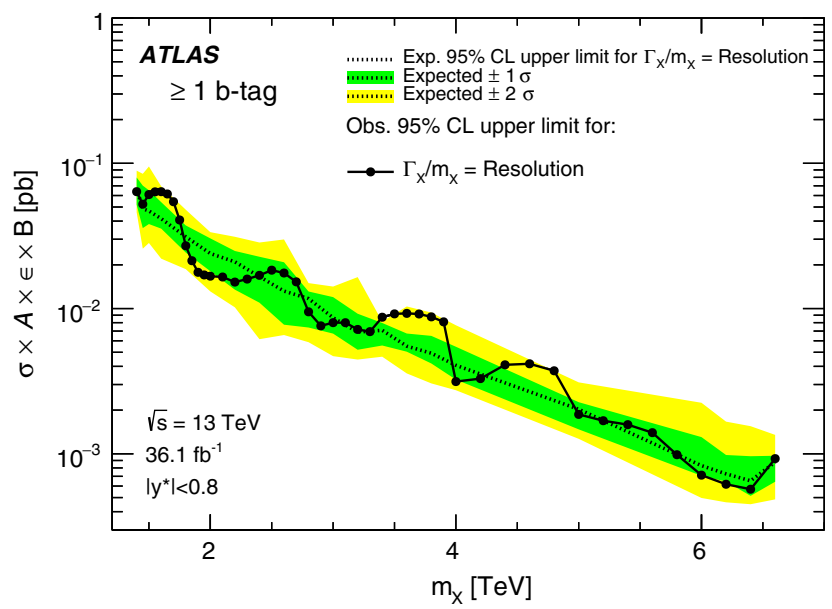

FIG. 8. Observed (filled circles) and expected (dotted line) $95 \%$ credibility-level upper limits on $\sigma \times A \times \epsilon \times \mathcal{B}(X \rightarrow b \bar{b})$, including kinematic acceptance and $b$-tagging efficiencies, for resonances with intrinsic width smaller than the detector resolution. The width of the Gaussian reconstructed shape is dominated by the dijet mass resolution. The plot shows the limits obtained from the high-mass inclusive $b$-jet selection.

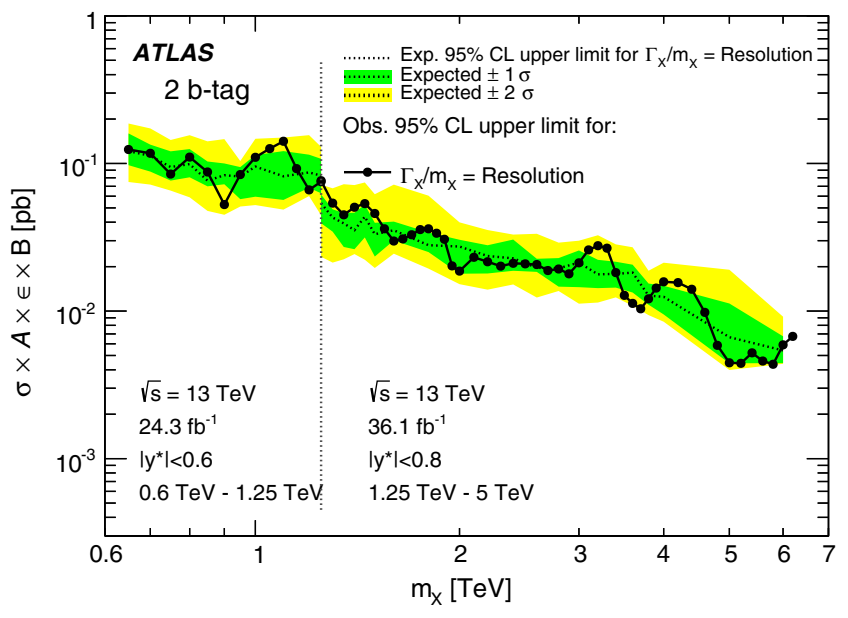

FIG. 9. Observed (filled circles) and expected (dotted line) $95 \%$ credibility-level upper limits on $\sigma \times A \times \epsilon \times \mathcal{B}(X \rightarrow b \bar{b})$, including kinematic acceptance and $b$-tagging efficiencies, for resonances with intrinsic width smaller than the detector resolution. The width of the Gaussian reconstructed shape is dominated by the dijet mass resolution. The plot shows the limits obtained from the combined low- and high-mass (separated by the vertical dotted line) two $b$-tags selection.

quoted on the product of the cross-section $\sigma$, acceptance $A$, selection efficiency $\epsilon$ and branching fraction $\mathcal{B}$ for a generic resonance with a reconstructed shape approximated by a Gaussian function, assuming a decay into two $b$-jets. A MC-based transfer matrix is used to fold in the detector effects. As the width is decreased from $15 \%$ to $0 \%$ of the mass, the cross-section limits improve, but at the same time the limits are more affected by statistical fluctuations of the data in a single bin as compared to wider signals. Figure 8 shows the limits for the inclusive $b$-jet selection when the intrinsic width is below the detector resolution. Figure 9 shows the corresponding limits for the low- and high-mass 2-b-tags selection.

\section{CONCLUSION}

Searches are performed for high-mass resonances in the dijet invariant mass spectrum with one or two jets identified as $b$-jets, using an integrated luminosity of up to $36.1 \mathrm{fb}^{-1}$ of proton-proton collisions with a center-of-mass energy of $\sqrt{s}=13 \mathrm{TeV}$ recorded by the ATLAS detector at the Large Hadron Collider. The search presented in this paper probes the mass range $0.57-5 \mathrm{TeV}$. No evidence of a significant excess of events above the expected Standard Model background is found.

Excited $b^{*}$-quarks with $b^{*} \rightarrow b g$ decays are excluded at 95\% C.L. for masses up to $2.6 \mathrm{TeV}$. New $Z^{\prime}$ gauge bosons are excluded in the sequential standard model (SSM) $Z^{\prime} \rightarrow$ $b \bar{b}$ model for masses up to $2.0 \mathrm{TeV}$, and excluded in the leptophobic $Z^{\prime} \rightarrow b \bar{b}$ model with SM-value couplings to quarks for masses up to $2.1 \mathrm{TeV}$, both at $95 \%$ C.L. Lastly, a $Z^{\prime}$ axial-vector dark-matter mediator with only $b$-quark 
couplings set to $g_{\mathrm{SM}}=0.25$ and axial DM couplings of $g_{\mathrm{DM}}=1.0$, is excluded at $95 \%$ C.L. for masses up to $2.1 \mathrm{TeV}$. Assuming $Z^{\prime}$ decays into all five quark flavors other than the top quark and $g_{\mathrm{SM}}=0.1$, masses up to $1.03 \mathrm{TeV}$ are excluded at 95\% C.L.

\section{ACKNOWLEDGMENTS}

We thank CERN for the very successful operation of the LHC, as well as the support staff from our institutions without whom ATLAS could not be operated efficiently. We acknowledge the support of ANPCyT, Argentina; YerPhI, Armenia; ARC, Australia; BMWFW and FWF, Austria; ANAS, Azerbaijan; SSTC, Belarus; CNPq and FAPESP, Brazil; NSERC, NRC and CFI, Canada; CERN; CONICYT, Chile; CAS, MOST and NSFC, China; COLCIENCIAS, Colombia; MSMT CR, MPO CR and VSC CR, Czech Republic; DNRF and DNSRC, Denmark; IN2P3-CNRS, CEA-DRF/IRFU, France; SRNSFG, Georgia; BMBF, HGF, and MPG, Germany; GSRT, Greece; RGC, Hong Kong SAR, China; ISF, I-CORE and Benoziyo Center, Israel; INFN, Italy; MEXT and JSPS, Japan; CNRST, Morocco; NWO, Netherlands; RCN, Norway; MNiSW and NCN, Poland; FCT, Portugal; MNE/IFA, Romania; MES of Russia and NRC KI, Russian Federation; JINR; MESTD, Serbia; MSSR, Slovakia; ARRS and MIZŠ, Slovenia; DST/NRF,
South Africa; MINECO, Spain; SRC and Wallenberg Foundation, Sweden; SERI, SNSF and Cantons of Bern and Geneva, Switzerland; MOST, Taiwan; TAEK, Turkey; STFC, United Kingdom; DOE and NSF, United States of America. In addition, individual groups and members have received support from BCKDF, the Canada Council, CANARIE, CRC, Compute Canada, FQRNT, and the Ontario Innovation Trust, Canada; EPLANET, ERC, ERDF, FP7, Horizon 2020 and Marie Skłodowska-Curie Actions, European Union; Investissements d'Avenir Labex and Idex, ANR, Région Auvergne and Fondation Partager le Savoir, France; DFG and AvH Foundation, Germany; Herakleitos, Thales and Aristeia programmes co-financed by EU-ESF and the Greek NSRF; BSF, GIF and Minerva, Israel; BRF, Norway; CERCA Programme Generalitat de Catalunya, Generalitat Valenciana, Spain; the Royal Society and Leverhulme Trust, United Kingdom. The crucial computing support from all WLCG partners is acknowledged gratefully, in particular from CERN, the ATLAS Tier-1 facilities at TRIUMF (Canada), NDGF (Denmark, Norway, Sweden), CC-IN2P3 (France), KIT/ GridKA (Germany), INFN-CNAF (Italy), NL-T1 (Netherlands), PIC (Spain), ASGC (Taiwan), RAL (UK) and BNL (USA), the Tier-2 facilities worldwide and large non-WLCG resource providers. Major contributors of computing resources are listed in Ref. [52].
[1] P. Langacker, The physics of heavy $Z^{\prime}$ gauge bosons, Rev. Mod. Phys. 81, 1199 (2009).

[2] C.-W. Chiang, T. Nomura, and K. Yagyu, Phenomenology of E6-inspired leptophobic $Z^{\prime}$ boson at the LHC, J. High Energy Phys. 05 (2014) 106.

[3] E. Eichten, I. Hinchliffe, K. Lane, and C. Quigg, Supercollider physics, Rev. Mod. Phys. 56, 579 (1984).

[4] U. Baur, I. Hinchliffe, and D. Zeppenfeld, Excited quark production at hadron colliders, Int. J. Mod. Phys. A 02, 1285 (1987).

[5] U. Baur, M. Spira, and P. M. Zerwas, Excited-quark and -lepton production at hadron colliders, Phys. Rev. D 42, 815 (1990).

[6] J. Abdallah et al., Simplified models for dark matter searches at the LHC, Phys. Dark Universe 9, 8 (2015).

[7] A. Boveia, Recommendations on presenting LHC searches for missing transverse energy signals using simplified $s$-channel models of dark matter, arXiv:1603.04156.

[8] M. Fairbairn, J. Heal, F. Kahlhoefer, and P. Tunney, Constraints on $Z^{\prime}$ models from LHC dijet searches and implications for dark matter, J. High Energy Phys. 09 (2016) 018 .

[9] D. Abercrombie et al., Dark matter benchmark models for early LHC run-2 searches: Report of the ATLAS/CMS dark matter forum, arXiv:1507.00966.
[10] M. Chala, F. Kahlhoefer, M. McCullough, G. Nardini, and K. Schmidt-Hoberg, Constraining Dark Sectors with Monojets and Dijets, J. High Energy Phys. 07 (2015) 089.

[11] UA1 Collaboration, Two-jet mass distributions at the CERN proton-antiproton Collider, Phys. Lett. B 209, 127 (1988).

[12] CDF Collaboration, Search for new particles decaying into dijets in proton-antiproton collisions at $\sqrt{s}=1.96 \mathrm{TeV}$, Phys. Rev. D 79, 112002 (2009).

[13] D0 Collaboration, Measurement of Dijet Angular Distributions at $\sqrt{s}=1.96 \mathrm{TeV}$ and Searches for Quark Compositeness and Extra Spatial Dimensions, Phys. Rev. Lett. 103, 191803 (2009).

[14] CMS Collaboration, Search for dijet resonances in protonproton collisions at $\sqrt{s}=13 \mathrm{TeV}$ and constraints on dark matter and other models, Phys. Lett. B 769, 520 (2017); Erratum, Phys. Lett. B 772, 882(E) (2017).

[15] ATLAS Collaboration, Search for new phenomena in dijet events using $37 \mathrm{fb}^{-1}$ of $p p$ collision data collected at $\sqrt{\mathrm{s}}=$ $13 \mathrm{TeV}$ with the ATLAS detector, Phys. Rev. D 96, 052004 (2017).

[16] ATLAS Collaboration, Search for low-mass dijet resonances using trigger-level jets with the ATLAS detector in $p p$ collisions at $\sqrt{s}=13 \mathrm{TeV}$ (2018), arXiv:1804.03496. 
[17] T. Jacques, A. Katz, E. Morgante, D. Racco, M. Rameez, and A. Riotto, Complementarity of DM searches in a consistent simplified model: the case of $Z^{\prime}$, J. High Energy Phys. 10 (2016) 071.

[18] Y. G. Kim, K. Y. Lee, C. B. Park, and S. Shin, Secluded singlet fermionic dark matter driven by the Fermi gammaray excess, Phys. Rev. D 93, 075023 (2016).

[19] CDF Collaboration, Search for New Particles Decaying to $b \bar{b}$ in $p \bar{p}$ Collisions at $\sqrt{s}=1.8 \mathrm{TeV}$, Phys. Rev. Lett. 82, 2038 (1999).

[20] CMS Collaboration, Search for narrow resonances and quantum black holes in inclusive and $b$-tagged dijet mass spectra from $p p$ collisions at $\sqrt{s}=7 \mathrm{TeV}$, J. High Energy Phys. 01 (2013) 013.

[21] CMS Collaboration, Search for Narrow Resonances in the $b$-Tagged Dijet Mass Spectrum in Proton-Proton Collisions at $\sqrt{s}=8 \mathrm{TeV}$, Phys. Rev. Lett. 120, 201801 (2018).

[22] ATLAS Collaboration, Search for resonances in the mass distribution of jet pairs with one or two jets identified as $b$ jets in proton-proton collisions at $\sqrt{s}=13 \mathrm{TeV}$ with the ATLAS detector, Phys. Lett. B 759, 229 (2016).

[23] ATLAS Collaboration, Search for Light Resonances Decaying into Boosted Quark Pairs and Produced in Association with a Photon or a Jet in Proton-proton Collisions at $\sqrt{S}=13$ Tev with the Atlas Detector, arXiv: 1801.08769 [Phys. Rev. Lett. (to be published)].

[24] ATLAS Collaboration, Search for new phenomena in the dijet mass distribution using $p p$ collision data at $\sqrt{s}=$ $8 \mathrm{TeV}$ with the ATLAS detector, Phys. Rev. D 91, 052007 (2015).

[25] ATLAS Collaboration, The ATLAS experiment at the CERN large Hadron Collider, J. Instrum. 3, S08003 (2008).

[26] ATLAS Collaboration, ATLAS insertable B-layer technical design report, Report Nos. CERN-LHCC-2010-013, ATLAS-TDR-19, CERN report, 2010, http://cds.cern.ch/ record/1291633.

[27] ATLAS Collaboration, ATLAS insertable B-layer technical design report addendum, Report No. ATLAS-TDR-019, CERN report, 2012 http://cds.cern.ch/record/1451888.

[28] T. Sjöstrand, S. Mrenna, and P.Z. Skands, A Brief Introduction to PYTHIA 8.1, Comput. Phys. Commun. 178, 852 (2008).

[29] ATLAS Collaboration, ATLAS Pythia 8 tunes to $7 \mathrm{TeV}$ data, Report No. ATL-PHYS-PUB-2014-021, CERN report, 2014, http://cdsweb.cern.ch/record/1966419.

[30] R. D. Ball et al. (NNPDF Collaboration), Parton distributions with LHC data, Nucl. Phys. B867, 244 (2013).

[31] D. J. Lange, The EvtGen particle decay simulation package, Nucl. Instrum. Methods Phys. Res., Sect. A 462, 152 (2001).

[32] ATLAS Collaboration, The ATLAS simulation infrastructure, Eur. Phys. J. C 70, 823 (2010).

[33] S. Agostinelli et al., GEANT4 - a simulation toolkit, Nucl. Instrum. Methods Phys. Res., Sect. A 506, 250 (2003).

[34] A. D. Martin, W. J. Stirling, R. S. Thorne, and G. Watt, Parton distributions for the LHC, Eur. Phys. J. C 63, 189 (2009).

[35] J. Alwall, R. Frederix, S. Frixione, V. Hirschi, F. Maltoni, O. Mattelaer, H.-S. Shao, T. Stelzer, P. Torrielli, and M. Zaro, The automated computation of tree-level and next-to-leading order differential cross sections, and their matching to parton shower simulations, J. High Energy Phys. 07 (2014) 079.

[36] A. Alloul, N. D. Christensen, C. Degrande, C. Duhr, and B. Fuks, FeynRules 2.0-A complete toolbox for tree-level phenomenology, Comput. Phys. Commun. 185, 2250 (2014).

[37] C. Degrande, Automatic evaluation of UV and R2 terms for beyond the Standard Model Lagrangians: A proof-ofprinciple, Comput. Phys. Commun. 197, 239 (2015).

[38] ATLAS Collaboration, Performance of the ATLAS trigger system in 2015, Eur. Phys. J. C 77, 317 (2017).

[39] ATLAS Collaboration, Topological cell clustering in the ATLAS calorimeters and its performance in LHC Run 1, Eur. Phys. J. C 77, 490 (2017).

[40] M. Cacciari, G. P. Salam, and G. Soyez, The anti- $k_{t}$ jet clustering algorithm, J. High Energy Phys. 04 (2008) 063.

[41] M. Cacciari, G. P. Salam, and G. Soyez, FastJet user manual, Eur. Phys. J. C 72, 1896 (2012).

[42] ATLAS Collaboration, Jet calibration and systematic uncertainties for jets reconstructed in the ATLAS detector at $\sqrt{s}=13 \mathrm{TeV}$, Report No. ATL-PHYS-PUB-2015-015, CERN report, 2015, https://cds.cern.ch/record/2037613.

[43] ATLAS Collaboration, Performance of $b$-jet identification in the ATLAS experiment, J. Instrum. 11, P04008 (2016).

[44] ATLAS Collaboration, Optimisation of the ATLAS $b$ tagging performance for the 2016 LHC Run, Report No. ATL-PHYS-PUB-2016-012, CERN report, 2016, https://cds.cern.ch/record/2160731.

[45] ATLAS Collaboration, Measurements of $b$-jet tagging efficiency with the ATLAS detector using $t \bar{t}$ events at $\sqrt{s}=13 \mathrm{TeV}$, arXiv:1805.01845.

[46] ATLAS Collaboration, Search for new phenomena in dijet mass and angular distributions from $p p$ collisions at $\sqrt{s}=$ $13 \mathrm{TeV}$ with the ATLAS detector, Phys. Lett. B 754, 302 (2016).

[47] G. Choudalakis, On hypothesis testing, trials factor, hypertests and the BumpHunter, in Proceedings of PHYSTAT 2011 Workshop on Statistical Issues Related to Discovery Claims in Search Experiments and Unfolding, CERN, Geneva, Switzerland, 2011, (CERN, Geneva, 2011), arXiv:1101.0390.

[48] ATLAS Collaboration, Jet energy scale measurements and their systematic uncertainties in proton-proton collisions at $\sqrt{s}=13 \mathrm{TeV}$ with the ATLAS detector, Phys. Rev. D 96, 072002 (2017).

[49] ATLAS Collaboration, Jet energy measurement and its systematic uncertainty in proton-proton collisions at $\sqrt{\mathrm{s}}=$ $7 \mathrm{TeV}$ with the ATLAS detector, Eur. Phys. J. C 75, 17 (2015).

[50] A. Caldwell, D. Kollar, and K. Kroninger, BAT: The bayesian analysis toolkit, Comput. Phys. Commun. 180, 2197 (2009).

[51] M. Baak, S. Gadatsch, R. Harrington, and W. Verkerke, Interpolation between multi-dimensional histograms using a new non-linear moment morphing method, Nucl. Instrum. Methods Phys. Res., Sect. A 771, 39 (2015).

[52] ATLAS Collaboration, ATLAS Computing Acknowledgements, Report No. ATL-GEN-PUB-2016-002, CERN report, 2016, https://cds.cern.ch/record/2202407. 
M. Aaboud ${ }^{34 d}$ G. Aad, ${ }^{99}$ B. Abbott, ${ }^{124}$ O. Abdinov, ${ }^{13, a}$ B. Abeloos, ${ }^{128}$ D. K. Abhayasinghe, ${ }^{91}$ S. H. Abidi, ${ }^{164}$ O. S. AbouZeid, ${ }^{143}$ N. L. Abraham, ${ }^{153}$ H. Abramowicz, ${ }^{158}$ H. Abreu, ${ }^{157}$ Y. Abulaiti, ${ }^{6}$ B. S. Acharya,${ }^{64 a, 64 b, b}$ S. Adachi, ${ }^{160}$ L. Adamczyk, ${ }^{81 \mathrm{a}} \mathrm{J}$. Adelman, ${ }^{119}$ M. Adersberger,${ }^{112}$ A. Adiguzel, ${ }^{12 \mathrm{c}, \mathrm{c}}$ T. Adye,${ }^{141}$ A. A. Affolder, ${ }^{143}$ Y. Afik, ${ }^{157}$ C. Agheorghiesei, ${ }^{27 \mathrm{c}}$ J. A. Aguilar-Saavedra, ${ }^{136 f, 136 \mathrm{a}}$ F. Ahmadov, ${ }^{77, \mathrm{~d}}$ G. Aielli, ${ }^{71 \mathrm{a}, 71 \mathrm{~b}}$ S. Akatsuka, ${ }^{83}$ T. P. A. Åkesson, ${ }^{94}$ E. Akilli, ${ }^{52}$ A. V. Akimov, ${ }^{108}$ G. L. Alberghi, ${ }^{23 b, 23 a}$ J. Albert, ${ }^{173}$ P. Albicocco,${ }^{49}$ M. J. Alconada Verzini, ${ }^{86}$ S. Alderweireldt, ${ }^{117}$ M. Aleksa, ${ }^{35}$ I. N. Aleksandrov, ${ }^{77}$ C. Alexa, ${ }^{27 b}$ T. Alexopoulos, ${ }^{10}$ M. Alhroob, ${ }^{124}$ B. Ali, ${ }^{138}$ G. Alimonti, ${ }^{66 a}$ J. Alison, ${ }^{36}$ S. P. Alkire, ${ }^{145}$ C. Allaire, ${ }^{128}$ B. M. M. Allbrooke, ${ }^{153}$ B. W. Allen, ${ }^{127}$ P. P. Allport, ${ }^{21}$ A. Aloisio, ${ }^{67 a, 67 b}$ A. Alonso, ${ }^{39}$ F. Alonso, ${ }^{86}$ C. Alpigiani, ${ }^{145}$ A. A. Alshehri, ${ }^{55}$ M. I. Alstaty, ${ }^{99}$ B. Alvarez Gonzalez,${ }^{35}$ D. Álvarez Piqueras, ${ }^{171}$ M. G. Alviggi, ${ }^{67 a, 67 b}$ B. T. Amadio, ${ }^{18}$ Y. Amaral Coutinho, ${ }^{78 b}$ L. Ambroz, ${ }^{131}$ C. Amelung, ${ }^{26}$ D. Amidei, ${ }^{103}$ S. P. Amor Dos Santos, ${ }^{136 a, 136 c}$ S. Amoroso, ${ }^{35}$ C. S. Amrouche,${ }^{52}$ C. Anastopoulos, ${ }^{146}$ L. S. Ancu, ${ }^{52}$ N. Andari, ${ }^{21}$ T. Andeen, ${ }^{11}$ C. F. Anders, ${ }^{59 b}$ J. K. Anders, ${ }^{20}$ K. J. Anderson, ${ }^{36}$ A. Andreazza, ${ }^{66 a, 66 b}$ V. Andrei,${ }^{59 a}$ C. R. Anelli, ${ }^{173}$ S. Angelidakis, ${ }^{37}$ I. Angelozzi, ${ }^{18}$ A. Angerami, ${ }^{38}$ A. V. Anisenkov, ${ }^{120 b, 120 a}$ A. Annovi, ${ }^{69 a}$ C. Antel, ${ }^{59 a}$ M. T. Anthony, ${ }^{146}$ M. Antonelli, ${ }^{49}$ D. J. A. Antrim, ${ }^{168}$ F. Anulli, ${ }^{70 a}$ M. Aoki, ${ }^{79}$ L. Aperio Bella, ${ }^{35}$ G. Arabidze,${ }^{104}$ Y. Arai,${ }^{79}$ J. P. Araque, ${ }^{16 \mathrm{a}}$ V. Araujo Ferraz, ${ }^{78 \mathrm{~b}}$ R. Araujo Pereira, ${ }^{78 \mathrm{~b}}$ A. T. H. Arce, ${ }^{47}$ R. E. Ardell, ${ }^{91}$ F. A. Arduh ${ }^{86}$ J-F. Arguin, ${ }^{107}$ S. Argyropoulos, ${ }^{75}$ A. J. Armbruster, ${ }^{35}$ L. J. Armitage, ${ }^{90}$ A Armstrong, ${ }^{168}$ O. Arnaez, ${ }^{164}$ H. Arnold, ${ }^{118}$ M. Arratia, ${ }^{31}$ O. Arslan, ${ }^{24}$ A. Artamonov, ${ }^{109, a}$ G. Artoni, ${ }^{131}$ S. Artz,${ }^{97}$ S. Asai,${ }^{160}$ N. Asbah, ${ }^{44}$ A. Ashkenazi, ${ }^{158}$ E. M. Asimakopoulou, ${ }^{169}$ L. Asquith, ${ }^{153}$ K. Assamagan, ${ }^{29}$ R. Astalos,${ }^{28 a}$ R. J. Atkin, ${ }^{32 a}$ M. Atkinson, ${ }^{170}$ N. B. Atlay, ${ }^{148}$ K. Augsten, ${ }^{138}$ G. Avolio, ${ }^{35}$ R. Avramidou, ${ }^{58 \mathrm{a}}$ B. Axen, ${ }^{18}$ M. K. Ayoub, ${ }^{15 \mathrm{a}}$ G. Azuelos, ${ }^{107, \mathrm{e}}$ A. E. Bas, ${ }^{59 \mathrm{a}}$ M. J. Baca, ${ }^{21}$ H. Bachacou, ${ }^{142}$ K. Bachas,${ }^{65 a, 65 b}$ M. Backes, ${ }^{131}$ P. Bagnaia, ${ }^{70 a, 70 b}$ M. Bahmani, ${ }^{82}$ H. Bahrasemani, ${ }^{149}$ A. J. Bailey, ${ }^{171}$ J. T. Baines, ${ }^{141}$ M. Bajic,${ }^{39}$ C. Bakalis, ${ }^{10}$ O. K. Baker, ${ }^{180}$ P. J. Bakker, ${ }^{118}$ D. Bakshi Gupta, ${ }^{93}$ E. M. Baldin, ${ }^{120 b, 120 a}$ P. Balek, ${ }^{177}$ F. Balli, ${ }^{142}$ W. K. Balunas, ${ }^{133}$ J. Balz, ${ }^{97}$ E. Banas, ${ }^{82}$ A. Bandyopadhyay, ${ }^{24}$ S. Banerjee, ${ }^{178, f}$ A. A. E. Bannoura, ${ }^{179}$ L. Barak, ${ }^{158}$ W. M. Barbe,${ }^{37}$ E. L. Barberio, ${ }^{102}$ D. Barberis, ${ }^{53 b, 53 a}$ M. Barbero, ${ }^{99}$ T. Barillari, ${ }^{113}$ M-S. Barisits, ${ }^{35}$ J. Barkeloo, ${ }^{127}$ T. Barklow, ${ }^{150}$ N. Barlow, ${ }^{31}$ R. Barnea, ${ }^{157}$ S. L. Barnes, ${ }^{58 \mathrm{c}}$ B. M. Barnett, ${ }^{141}$ R. M. Barnett, ${ }^{18}$ Z. Barnovska-Blenessy, ${ }^{58 \mathrm{a}}$ A. Baroncelli, ${ }^{72 \mathrm{a}}$ G. Barone, ${ }^{26}$ A. J. Barr, ${ }^{131}$ L. Barranco Navarro, ${ }^{171}$ F. Barreiro, ${ }^{96}$ J. Barreiro Guimarães da Costa, ${ }^{15 a}$ R. Bartoldus, ${ }^{150}$ A. E. Barton, ${ }^{87}$ P. Bartos, ${ }^{28 a}$ A. Basalaev, ${ }^{134}$ A. Bassalat, ${ }^{128}$ R. L. Bates, ${ }^{55}$ S. J. Batista, ${ }^{164}$ S. Batlamous, ${ }^{34 \mathrm{e}}$ J. R. Batley, ${ }^{31}$ M. Battaglia, ${ }^{143}$ M. Bauce, ${ }^{70 a, 70 b}$ F. Bauer,${ }^{142}$ K. T. Bauer, ${ }^{168}$ H. S. Bawa ${ }^{150, g}$ J. B. Beacham, ${ }^{122}$ M. D. Beattie,${ }^{87}$ T. Beau, ${ }^{132}$ P. H. Beauchemin,${ }^{167}$ P. Bechtle,${ }^{24}$ H. C. Beck, ${ }^{51}$ H. P. Beck, ${ }^{20, \mathrm{~h}}$ K. Becker, ${ }^{50}$ M. Becker, ${ }^{97}$ C. Becot, ${ }^{44}$ A. Beddall, ${ }^{12 \mathrm{~d}}$ A. J. Beddall, ${ }^{12 \mathrm{a}}$ V. A. Bednyakov, ${ }^{77}$ M. Bedognetti, ${ }^{118}$ C. P. Bee, ${ }^{152}$ T. A. Beermann, ${ }^{35}$ M. Begalli, ${ }^{78 b}$ M. Begel,${ }^{29}$ A. Behera, ${ }^{152}$ J. K. Behr, ${ }^{44}$ A. S. Bell, ${ }^{92}$ G. Bella, ${ }^{158}$ L. Bellagamba, ${ }^{23 b}$ A. Bellerive, ${ }^{33}$ M. Bellomo, ${ }^{157}$ P. Bellos, ${ }^{9}$ K. Belotskiy, ${ }^{110}$ N. L. Belyaev, ${ }^{110}$ O. Benary, ${ }^{15, a}$ D. Benchekroun, ${ }^{34 a}$ M. Bender, ${ }^{12}$ N. Benekos, ${ }^{10}$ Y. Benhammou, ${ }^{158}$ E. Benhar Noccioli, ${ }^{180}$ J. Benitez,${ }^{75}$ D. P. Benjamin,${ }^{47}$ M. Benoit, ${ }^{52}$ J. R. Bensinger, ${ }^{26}$ S. Bentvelsen, ${ }^{118}$ L. Beresford, ${ }^{131}$ M. Beretta, ${ }^{49}$ D. Berge, ${ }^{44}$ E. Bergeaas Kuutmann, ${ }^{169}$ N. Berger, ${ }^{5}$ L. J. Bergsten, ${ }^{26}$ J. Beringer, ${ }^{18}$ S. Berlendis, ${ }^{7}$ N. R. Bernard, ${ }^{100}$ G. Bernardi, ${ }^{132}$ C. Bernius, ${ }^{150}$ F. U. Bernlochner, ${ }^{24}$ T. Berry, ${ }^{91}$ P. Berta, ${ }^{97}$ C. Bertella, ${ }^{15 a}$ G. Bertoli, ${ }^{43 a}{ }^{43 b}$ I. A. Bertram, ${ }^{87}$ G. J. Besjes,${ }^{39}$ O. Bessidskaia Bylund, ${ }^{4 a, 43 b}$ M. Bessner, ${ }^{44}$ N. Besson, ${ }^{142}$ A. Bethani, ${ }^{98}$ S. Bethke, ${ }^{113}$ A. Betti, ${ }^{24}$ A. J. Bevan,${ }^{90}$ J. Beyer, ${ }^{113}$ R. M. B. Bianchi, ${ }^{135}$ O. Biebel, ${ }^{112}$ D. Biedermann, ${ }^{19}$ R. Bielski, ${ }^{98}$ K. Bierwagen, ${ }^{97}$ N. V. Biesuz, ${ }^{69 a, 69 b}$ M. Biglietti, ${ }^{72 a}$

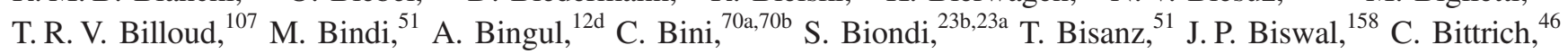
D. M. Bjergaard, ${ }^{47}$ J. E. Black, ${ }^{150}$ K. M. Black ${ }^{25}$ R. E. Blair, ${ }^{6}$ T. Blazek, ${ }^{28 a}$ I. Bloch, ${ }^{44}$ C. Blocker, ${ }^{26}$ A. Blue,${ }^{55}$ U. Blumenschein, ${ }^{90}$ Dr. Blunier, ${ }^{144 a}$ G. J. Bobbink, ${ }^{118}$ V. S. Bobrovnikov, ${ }^{120 b, 120 a}$ S. S. Bocchetta, ${ }^{94}$ A. Bocci, ${ }^{47}$ D. Boerner, ${ }^{179}$ D. Bogavac, ${ }^{112}$ A. G. Bogdanchikov, ${ }^{120 b, 120 a}$ C. Bohm, ${ }^{43 a}$ V. Boisvert, ${ }^{91}$ P. Bokan, ${ }^{169, \mathrm{i}}$ T. Bold, ${ }^{81 a}$ A. S. Boldyrev, ${ }^{111}$ A. E. Bolz, ${ }^{59 b}$ M. Bomben, ${ }^{132}$ M. Bona,${ }^{90}$ J. S. Bonilla, ${ }^{127}$ M. Boonekamp, ${ }^{142}$ A. Borisov, ${ }^{140}$ G. Borissov, ${ }^{87}$ J. Bortfeldt, ${ }^{35}$ D. Bortoletto, ${ }^{131}$ V. Bortolotto, ${ }^{71 a, 61 b, 61 c, 71 b}$ D. Boscherini, ${ }^{23 b}$ M. Bosman, ${ }^{14}$ J. D. Bossio Sola, ${ }^{30}$ K. Bouaouda, ${ }^{34 a}$ J. Boudreau, ${ }^{135}$ E. V. Bouhova-Thacker, ${ }^{87}$ D. Boumediene, ${ }^{37}$ C. Bourdarios, ${ }^{128}$ S. K. Boutle, ${ }^{55}$ A. Boveia, ${ }^{122}$ J. Boyd,${ }^{35}$ I. R. Boyko, ${ }^{77}$ A. J. Bozson, ${ }^{91}$ J. Bracinik, ${ }^{21}$ N. Brahimi, ${ }^{99}$ A. Brandt, ${ }^{8}$ G. Brandt,${ }^{179}$ O. Brandt, ${ }^{59 a}$ F. Braren, ${ }^{44}$ U. Bratzler, ${ }^{161}$ B. Brau, ${ }^{100}$ J. E. Brau, ${ }^{127}$ W. D. Breaden Madden, ${ }^{55}$ K. Brendlinger, ${ }^{44}$ A. J. Brennan, ${ }^{102}$ L. Brenner, ${ }^{44}$ R. Brenner, ${ }^{169}$ S. Bressler, ${ }^{177}$ B. Brickwedde, ${ }^{97}$ D. L. Briglin, ${ }^{21}$ D. Britton, ${ }^{55}$ D. Britzger, ${ }^{59}$ I. Brock, ${ }^{24}$ R. Brock, ${ }^{104}$ G. Brooijmans, ${ }^{38}$ T. Brooks, ${ }^{91}$ W. K. Brooks,,${ }^{144 b}$ E. Brost,${ }^{119}$ J. H Broughton, ${ }^{21}$ P. A. Bruckman de Renstrom,${ }^{82}$ D. Bruncko, ${ }^{28 b}$ A. Bruni, ${ }^{23 b}$ G. Bruni, ${ }^{23 b}$ L. S. Bruni, ${ }^{118}$ S. Bruno, ${ }^{71 a, 71 b}$ B. H. Brunt, ${ }^{31}$ M. Bruschi, ${ }^{23 b}$ N. Bruscino, ${ }^{135}$ P. Bryant,${ }^{36}$ L. Bryngemark ${ }^{44}$ T. Buanes, ${ }^{17}$ Q. Buat,${ }^{35}$ P. Buchholz, ${ }^{148}$ A. G. Buckley, ${ }^{55}$ I. A. Budagov,${ }^{77}$ F. Buehrer,${ }^{50}$ M. K. Bugge, ${ }^{130}$ O. Bulekov, ${ }^{110}$ D. Bullock, ${ }^{8}$ T. J. Burch, ${ }^{119}$ S. Burdin, ${ }^{88}$ C. D. Burgard, ${ }^{118}$ A. M. Burger, ${ }^{5}$ B. Burghgrave, ${ }^{119}$ 
K. Burka, ${ }^{82}$ S. Burke, ${ }^{141}$ I. Burmeister, ${ }^{45}$ J. T. P. Burr, ${ }^{131}$ D. Büscher, ${ }^{50}$ V. Büscher, ${ }^{97}$ E. Buschmann, ${ }^{51}$ P. Bussey, ${ }^{55}$ J. M. Butler, ${ }^{25}$ C. M. Buttar, ${ }^{55}$ J. M. Butterworth, ${ }^{92}$ P. Butti, ${ }^{35}$ W. Buttinger, ${ }^{35}$ A. Buzatu, ${ }^{155}$ A. R. Buzykaev, ${ }^{120 b, 120 a}$ G. Cabras, ${ }^{23 b, 23 a}$ S. Cabrera Urbán, ${ }^{171}$ D. Caforio, ${ }^{138}$ H. Cai, ${ }^{170}$ V. M. M. Cairo, ${ }^{2}$ O. Cakir, ${ }^{4 a}$ N. Calace, ${ }^{52}$ P. Calafiura, ${ }^{18}$ A. Calandri, ${ }^{99}$ G. Calderini, ${ }^{132}$ P. Calfayan, ${ }^{63}$ G. Callea, ${ }^{40 b, 40 a}$ L. P. Caloba, ${ }^{78 b}$ S. Calvente Lopez, ${ }^{96}$ D. Calvet, ${ }^{37}$ S. Calvet, ${ }^{37}$ T. P. Calvet, ${ }^{152}$ M. Calvetti, ${ }^{69 a, 69 b}$ R. Camacho Toro, ${ }^{132}$ S. Camarda, ${ }^{35}$ P. Camarri, ${ }^{71 a, 71 b}$ D. Cameron, ${ }^{130}$

R. Caminal Armadans, ${ }^{100}$ C. Camincher, ${ }^{35}$ S. Campana, ${ }^{35}$ M. Campanelli, ${ }^{92}$ A. Camplani, ${ }^{39}$ A. Campoverde, ${ }^{148}$ V. Canale ${ }^{67 \mathrm{a}, 67 \mathrm{~b}}$ M. Cano Bret, ${ }^{58 \mathrm{c}}$ J. Cantero, ${ }^{125}$ T. Cao, ${ }^{158}$ Y. Cao, ${ }^{170}$ M. D. M. Capeans Garrido, ${ }^{35}$ I. Caprini, ${ }^{27 b}$ M. Caprini, ${ }^{27 b}$ M. Capua, ${ }^{40 b, 40 a}$ R. M. Carbone, ${ }^{38}$ R. Cardarelli, ${ }^{71 a}$ F. C. Cardillo, ${ }^{50}$ I. Carli, ${ }^{139}$ T. Carli, ${ }^{35}$ G. Carlino, ${ }^{67 a}$ B. T. Carlson, ${ }^{135}$ L. Carminati, ${ }^{66 a, 66 b}$ R. M. D. Carney, ${ }^{43 a, 43 b}$ S. Caron, ${ }^{117}$ E. Carquin, ${ }^{144 b}$ S. Carrá, ${ }^{66 a, 66 b}$ G. D. Carrillo-Montoya, ${ }^{35}$ D. Casadei, ${ }^{32 b}$ M. P. Casado, ${ }^{14, j}$ A. F. Casha, ${ }^{164}$ M. Casolino, ${ }^{14}$ D. W. Casper, ${ }^{168}$ R. Castelijn, ${ }^{118}$ F. L. Castillo, ${ }^{171}$ V. Castillo Gimenez, ${ }^{171}$ N. F. Castro, ${ }^{136 a, 136 e}$ A. Catinaccio, ${ }^{35}$ J. R. Catmore, ${ }^{130}$ A. Cattai, ${ }^{35}$ J. Caudron, ${ }^{24}$ V. Cavaliere, ${ }^{29}$ E. Cavallaro, ${ }^{14}$ D. Cavalli, ${ }^{66 a}$ M. Cavalli-Sforza, ${ }^{14}$ V. Cavasinni, ${ }^{69 a, 69 b}$ E. Celebi, ${ }^{12 b}$ F. Ceradini, ${ }^{72 a, 72 b}$ L. Cerda Alberich, ${ }^{171}$ A. S. Cerqueira, ${ }^{78 a}$ A. Cerri, ${ }^{153}$ L. Cerrito, ${ }^{71 a, 71 b}$ F. Cerutti, ${ }^{18}$ A. Cervelli, ${ }^{23 b, 23 a}$ S. A. Cetin, ${ }^{12 b}$ A. Chafaq, ${ }^{34 a}$ D Chakraborty, ${ }^{119}$ S. K. Chan, ${ }^{57}$ W. S. Chan, ${ }^{118}$ Y. L. Chan, ${ }^{61 a}$ P. Chang, ${ }^{170}$ J. D. Chapman, ${ }^{31}$ D. G. Charlton, ${ }^{21}$ C. C. Chau, ${ }^{33}$ C. A. Chavez Barajas, ${ }^{153}$ S. Che, ${ }^{122}$ A. Chegwidden, ${ }^{104}$ S. Chekanov, ${ }^{6}$ S. V. Chekulaev, ${ }^{165 a}$ G. A. Chelkov, ${ }^{77, k}$ M. A. Chelstowska, ${ }^{35}$ C. Chen, ${ }^{58 a}$ C. H. Chen, ${ }^{76}$ H. Chen, ${ }^{29}$ J. Chen, ${ }^{58 a}$ J. Chen, ${ }^{38}$ S. Chen, ${ }^{133}$ S. J. Chen, ${ }^{15 c}$ X. Chen, ${ }^{15 b, 1}$ Y. Chen, ${ }^{80}$ Y-H. Chen, ${ }^{44}$ H. C. Cheng, ${ }^{103}$ H. J. Cheng, ${ }^{15 d}$ A. Cheplakov, ${ }^{77}$ E. Cheremushkina, ${ }^{140}$ R. Cherkaoui El Moursli, ${ }^{34 \mathrm{e}}$ E. Cheu, ${ }^{7}$ K. Cheung, ${ }^{62}$ L. Chevalier, ${ }^{142}$ V. Chiarella, ${ }^{49}$ G. Chiarelli, ${ }^{69 a}$ G. Chiodini, ${ }^{65 a}$ A. S. Chisholm, ${ }^{35}$ A. Chitan, ${ }^{27 b}$ I. Chiu, ${ }^{160}$ Y. H. Chiu, ${ }^{173}$ M. V. Chizhov, ${ }^{77}$ K. Choi, ${ }^{63}$ A. R. Chomont, ${ }^{128}$ S. Chouridou, ${ }^{159}$ Y. S. Chow, ${ }^{18}$

V. Christodoulou, ${ }^{92}$ M. C. Chu, ${ }^{61 a}$ J. Chudoba, ${ }^{137}$ A. J. Chuinard, ${ }^{101}$ J. J. Chwastowski, ${ }^{82}$ L. Chytka, ${ }^{126}$ D. Cinca, ${ }^{45}$ V. Cindro, ${ }^{89}$ I. A. Cioară, ${ }^{24}$ A. Ciocio, ${ }^{18}$ F. Cirotto, ${ }^{67 a, 67 b}$ Z. H. Citron, ${ }^{177}$ M. Citterio, ${ }^{66 a}$ A. Clark, ${ }^{52}$ M. R. Clark, ${ }^{38}$ P. J. Clark, ${ }^{48}$ C. Clement, ${ }^{43 a, 43 b}$ Y. Coadou, ${ }^{99}$ M. Cobal, ${ }^{64 a, 64 c}$ A. Coccaro, ${ }^{53 b, 53 a}$ J. Cochran, ${ }^{76}$ A. E. C. Coimbra, ${ }^{177}$ L. Colasurdo, ${ }^{117}$ B. Cole, ${ }^{38}$ A. P. Colijn, ${ }^{118}$ J. Collot, ${ }^{56}$ P. Conde Muiño, ${ }^{136 a, 136 b}$ E. Coniavitis, ${ }^{50}$ S. H. Connell, ${ }^{32 b}$ I. A. Connelly, ${ }^{98}$ S. Constantinescu, ${ }^{27 b}$ F. Conventi, ${ }^{67 a, m}$ A. M. Cooper-Sarkar, ${ }^{131}$ F. Cormier, ${ }^{172}$ K. J. R. Cormier, ${ }^{164}$ M. Corradi, ${ }^{70 a, 70 b}$ E. E. Corrigan, ${ }^{94}$ F. Corriveau, ${ }^{101, n}$ A. Cortes-Gonzalez, ${ }^{35}$ M. J. Costa, ${ }^{171}$ D. Costanzo, ${ }^{146}$ G. Cottin, ${ }^{31}$ G. Cowan, ${ }^{91}$ B. E. Cox, ${ }^{98}$ J. Crane, ${ }^{98}$ K. Cranmer, ${ }^{121}$ S. J. Crawley, ${ }^{55}$ R. A. Creager, ${ }^{133}$ G. Cree, ${ }^{33}$ S. Crépé-Renaudin, ${ }^{56}$ F. Crescioli, ${ }^{132}$ M. Cristinziani, ${ }^{24}$ V. Croft, ${ }^{121}$ G. Crosetti, ${ }^{40 b, 40 a}$ A. Cueto, ${ }^{96}$ T. Cuhadar Donszelmann, ${ }^{146}$ A. R. Cukierman, ${ }^{150}$ M. Curatolo, ${ }^{49}$ J. Cúth, ${ }^{97}$ S. Czekierda, ${ }^{82}$ P. Czodrowski, ${ }^{35}$ M. J. Da Cunha Sargedas De Sousa, ${ }^{58 b, 136 b}$ C. Da Via, ${ }^{98}$ W. Dabrowski, ${ }^{81 a}$ T. Dado, ${ }^{28 a, i}$ S. Dahbi, ${ }^{34 \mathrm{e}}$ T. Dai, ${ }^{103}$ F. Dallaire, ${ }^{107}$ C. Dallapiccola, ${ }^{100}$ M. Dam, ${ }^{39}$ G. D'amen, ${ }^{23 b, 23 a}$ J. Damp, ${ }^{97}$ J. R. Dandoy, ${ }^{133}$ M. F. Daneri, ${ }^{30}$ N. P. Dang, ${ }^{178, f}$ N. D Dann, ${ }^{98}$ M. Danninger, ${ }^{172}$ V. Dao, ${ }^{35}$ G. Darbo, ${ }^{53 \mathrm{~b}}$ S. Darmora, ${ }^{8}$ O. Dartsi, ${ }^{5}$ A. Dattagupta, ${ }^{127}$ T. Daubney, ${ }^{44}$ S. D' Auria, ${ }^{55}$ W. Davey, ${ }^{24}$ C. David, ${ }^{44}$ T. Davidek, ${ }^{139}$ D. R. Davis, ${ }^{47}$ E. Dawe, ${ }^{102}$ I. Dawson, ${ }^{146}$ K. De ${ }^{8}$ R. De Asmundis, ${ }^{67 a}$ A. De Benedetti, ${ }^{124}$ S. De Castro, ${ }^{23 b, 23 a}$

S. De Cecco, ${ }^{70 a, 70 b}$ N. De Groot, ${ }^{117}$ P. de Jong, ${ }^{118}$ H. De la Torre, ${ }^{104}$ F. De Lorenzi, ${ }^{76}$ A. De Maria, ${ }^{51, o}$ D. De Pedis, ${ }^{70 a}$ A. De Salvo, ${ }^{70 a}$ U. De Sanctis, ${ }^{71 a, 71 b}$ A. De Santo, ${ }^{153}$ K. De Vasconcelos Corga, ${ }^{99}$ J. B. De Vivie De Regie, ${ }^{128}$ C. Debenedetti, ${ }^{143}$ D. V. Dedovich, ${ }^{77}$ N. Dehghanian, ${ }^{3}$ M. Del Gaudio, ${ }^{40 b, 40 a}$ J. Del Peso, ${ }^{96}$ D. Delgove, ${ }^{128}$ F. Deliot, ${ }^{142}$ C. M. Delitzsch, ${ }^{7}$ M. Della Pietra, ${ }^{67 a, 67 b}$ D. Della Volpe, ${ }^{52}$ A. Dell' Acqua, ${ }^{35}$ L. Dell' Asta, ${ }^{25}$ M. Delmastro, ${ }^{5}$ C. Delporte, ${ }^{128}$ P. A. Delsart, ${ }^{56}$ D. A. DeMarco, ${ }^{164}$ S. Demers, ${ }^{180}$ M. Demichev, ${ }^{77}$ S. P. Denisov, ${ }^{140}$ D. Denysiuk, ${ }^{118}$ L. D'Eramo, ${ }^{132}$ D. Derendarz, ${ }^{82}$ J. E. Derkaoui, ${ }^{34 d}$ F. Derue, ${ }^{132}$ P. Dervan, ${ }^{88}$ K. Desch, ${ }^{24}$ C. Deterre, ${ }^{44}$ K. Dette, ${ }^{164}$ M. R. Devesa, ${ }^{30}$ P. O. Deviveiros, ${ }^{35}$ A. Dewhurst, ${ }^{141}$ S. Dhaliwal, ${ }^{26}$ F. A. Di Bello, ${ }^{52}$ A. Di Ciaccio, ${ }^{71 a, 71 b}$ L. Di Ciaccio, ${ }^{5}$ W. K. Di Clemente, ${ }^{133}$ C. Di Donato, ${ }^{67 a, 67 b}$ A. Di Girolamo, ${ }^{35}$ B. Di Micco, ${ }^{72 a, 72 b}$ R. Di Nardo, ${ }^{35}$ K. F. Di Petrillo, ${ }^{57}$ A. Di Simone, ${ }^{50}$ R. Di Sipio, ${ }^{164}$ D. Di Valentino, ${ }^{33}$ C. Diaconu, ${ }^{99}$ M. Diamond, ${ }^{164}$ F. A. Dias, ${ }^{39}$ T. Dias Do Vale, ${ }^{136 a}$ M. A. Diaz, ${ }^{14 a}$ J. Dickinson, ${ }^{18}$ E. B. Diehl, ${ }^{103}$ J. Dietrich, ${ }^{19}$ S. Díez Cornell, ${ }^{44}$ A. Dimitrievska, ${ }^{18}$ J. Dingfelder, ${ }^{24}$ F. Dittus, ${ }^{35}$ F. Djama, ${ }^{99}$ T. Djobava, ${ }^{156 b}$ J. I. Djuvsland, ${ }^{59}$ M. A. B. Do Vale, ${ }^{78 c}$ M. Dobre, ${ }^{27 b}$ D. Dodsworth, ${ }^{26}$ C. Doglioni, ${ }^{94}$ J. Dolejsi, ${ }^{139}$ Z. Dolezal, ${ }^{139}$ M. Donadelli, ${ }^{78 d}$ J. Donini, ${ }^{37}$ A. D'onofrio, ${ }^{90}$ M. D'Onofrio, ${ }^{88}$ J. Dopke, $^{141}$ A. Doria, ${ }^{67 a}$ M. T. Dova, ${ }^{86}$ A. T. Doyle, ${ }^{55}$ E. Drechsler, ${ }^{51}$ E. Dreyer, ${ }^{149}$ T. Dreyer, ${ }^{51}$ M. Dris, ${ }^{10}$ Y. Du, ${ }^{58 b}$ J. Duarte-Campderros, ${ }^{158}$ F. Dubinin, ${ }^{108}$ M. Dubovsky, ${ }^{28 a}$ A. Dubreuil, ${ }^{52}$ E. Duchovni, ${ }^{177}$ G. Duckeck, ${ }^{112}$ A. Ducourthial, ${ }^{132}$ O. A. Ducu, ${ }^{107, p}$ D. Duda, ${ }^{113}$ A. Dudarev, ${ }^{35}$ A. C. Dudder, ${ }^{97}$ E. M. Duffield, ${ }^{18}$ L. Duflot, ${ }^{128}$ M. Dührssen, ${ }^{35}$ C. Dülsen, ${ }^{179}$ M. Dumancic, ${ }^{177}$ A. E. Dumitriu, ${ }^{27 b, q}$ A. K. Duncan, ${ }^{55}$ M. Dunford, ${ }^{59 a}$ A. Duperrin, ${ }^{99}$ H. Duran Yildiz, ${ }^{4 a}$ M. Düren, ${ }^{54}$ A. Durglishvili, ${ }^{156 b}$ D. Duschinger, ${ }^{46}$ B. Dutta, ${ }^{44}$ D. Duvnjak, ${ }^{1}$ M. Dyndal, ${ }^{44}$ S. Dysch, ${ }^{98}$ B. S. Dziedzic, ${ }^{82}$ C. Eckardt, ${ }^{44}$ K. M. Ecker, ${ }^{113}$ 
R. C. Edgar ${ }^{103}$ T. Eifert, ${ }^{35}$ G. Eigen, ${ }^{17}$ K. Einsweiler, ${ }^{18}$ T. Ekelof, ${ }^{169}$ M. El Kacimi, ${ }^{34 c}$ R. El Kosseifi, ${ }^{99}$ V. Ellajosyula, ${ }^{99}$ M. Ellert, ${ }^{169}$ F. Ellinghaus, ${ }^{179}$ A. A. Elliot, ${ }^{90}$ N. Ellis, ${ }^{35}$ J. Elmsheuser, ${ }^{29}$ M. Elsing, ${ }^{35}$ D. Emeliyanov, ${ }^{141}$ Y. Enari, ${ }^{160}$ J. S. Ennis, ${ }^{175}$ M. B. Epland, ${ }^{47}$ J. Erdmann, ${ }^{45}$ A. Ereditato, ${ }^{20}$ S. Errede,${ }^{170}$ M. Escalier,${ }^{128}$ C. Escobar,${ }^{171}$ B. Esposito, ${ }^{49}$ O. Estrada Pastor, ${ }^{171}$ A. I. Etienvre, ${ }^{142}$ E. Etzion, ${ }^{158}$ H. Evans, ${ }^{63}$ A. Ezhilov, ${ }^{134}$ M. Ezzi, ${ }^{34 \mathrm{e}}$ F. Fabbri, ${ }^{55}$ L. Fabbri, ${ }^{23 b, 23 a}$ V. Fabiani, ${ }^{117}$ G. Facini, ${ }^{92}$ R. M. Faisca Rodrigues Pereira, ${ }^{136 a}$ R. M. Fakhrutdinov, ${ }^{140}$ S. Falciano, ${ }^{70 a}$ P. J. Falke,,${ }^{5}$ S. Falke, ${ }^{5}$ J. Faltova, ${ }^{139}$ Y. Fang, ${ }^{15 a}$ M. Fanti, ${ }^{66 a, 66 b}$ A. Farbin, ${ }^{8}$ A. Farilla, ${ }^{72 a}$ E. M. Farina, ${ }^{68 a, 68 b}$ T. Farooque,${ }^{104}$ S. Farrell, ${ }^{18}$

S. M. Farrington, ${ }^{175}$ P. Farthouat, ${ }^{35}$ F. Fassi, ${ }^{34 \mathrm{e}}$ P. Fassnacht,${ }^{35}$ D. Fassouliotis, ${ }^{9}$ M. Faucci Giannelli, ${ }^{48}$ A. Favareto, ${ }^{53 b, 53 a}$ W. J. Fawcett, ${ }^{52}$ L. Fayard, ${ }^{128}$ O. L. Fedin, ${ }^{134, \mathrm{r}}$ W. Fedorko, ${ }^{172}$ M. Feickert, ${ }^{41}$ S. Feigl, ${ }^{130}$ L. Feligioni, ${ }^{99}$ C. Feng, ${ }^{58 b}$ E. J. Feng, ${ }^{35}$ M. Feng, ${ }^{47}$ M. J. Fenton, ${ }^{55}$ A. B. Fenyuk, ${ }^{140}$ L. Feremenga, ${ }^{8}$ J. Ferrando, ${ }^{44}$ A. Ferrari, ${ }^{169}$ P. Ferrari, ${ }^{118}$ R. Ferrari, ${ }^{68 a}$ D. E. Ferreira de Lima, ${ }^{59 b}$ A. Ferrer, ${ }^{171}$ D. Ferrere, ${ }^{52}$ C. Ferretti, ${ }^{103}$ F. Fiedler, ${ }^{97}$ A. Filipčič,${ }^{89}$ F. Filthaut, ${ }^{117}$ K. D. Finelli, ${ }^{25}$ M. C. N. Fiolhais, ${ }^{136 a, 136 c, s}$ L. Fiorini, ${ }^{171}$ C. Fischer, ${ }^{14}$ W. C. Fisher ${ }^{104}$ N. Flaschel,${ }^{44}$ I. Fleck, ${ }^{148}$ P. Fleischmann, ${ }^{103}$ R. R. M. Fletcher, ${ }^{133}$ T. Flick, ${ }^{179}$ B. M. Flierl, ${ }^{12}$ L. M. Flores, ${ }^{133}$ L. R. Flores Castillo, ${ }^{61 a}$ N. Fomin,${ }^{17}$ G. T. Forcolin, ${ }^{98}$ A. Formica, ${ }^{142}$ F. A. Förster, ${ }^{14}$ A. C. Forti, ${ }^{98}$ A. G. Foster, ${ }^{21}$ D. Fournier, ${ }^{128}$ H. Fox, ${ }^{87}$ S. Fracchia, ${ }^{146}$ P. Francavilla, ${ }^{69 a, 69 b}$ M. Franchini, ${ }^{23 b, 23 a}$ S. Franchino, ${ }^{59 a}$ D. Francis,${ }^{35}$ L. Franconi, ${ }^{130}$ M. Franklin, ${ }^{57}$ M. Frate, ${ }^{168}$ M. Fraternali, ${ }^{68 \mathrm{a}, 68 \mathrm{~b}}$ D. Freeborn, ${ }^{92}$ S. M. Fressard-Batraneanu, ${ }^{35}$ B. Freund, ${ }^{107}$ W. S. Freund, ${ }^{78 b}$ D. Froidevaux, ${ }^{35}$ J. A. Frost ${ }^{131}$ C. Fukunaga, ${ }^{161}$ T. Fusayasu, ${ }^{114}$ J. Fuster, ${ }^{171}$ O. Gabizon, ${ }^{157}$ A. Gabrielli, ${ }^{23 b, 23 a}$ A. Gabrielli, ${ }^{18}$ G. P. Gach, ${ }^{81 a}$ S. Gadatsch, ${ }^{52}$ P. Gadow, ${ }^{113}$ G. Gagliardi, ${ }^{53 b, 53 a}$ L. G. Gagnon, ${ }^{107}$ C. Galea, ${ }^{27 b}$ B. Galhardo, ${ }^{136 a, 136 c}$ E. J. Gallas, ${ }^{131}$ B. J. Gallop ${ }^{141}$ P. Gallus, ${ }^{138}$ G. Galster ${ }^{39}$ R. Gamboa Goni,${ }^{90}$ K. K. Gan,${ }^{122}$ S. Ganguly, ${ }^{177}$ Y. Gao, ${ }^{88}$ Y. S. Gao, ${ }^{150, g}$ C. García, ${ }^{171}$ J. E. García Navarro, ${ }^{171}$ J. A. García Pascual, ${ }^{15 a}$ M. Garcia-Sciveres, ${ }^{18}$ R. W. Gardner, ${ }^{36}$ N. Garelli, ${ }^{150}$ V. Garonne, ${ }^{130}$ K. Gasnikova, ${ }^{44}$ A. Gaudiello, ${ }^{53 b, 53 a}$ G. Gaudio, ${ }^{68 a}$ I. L. Gavrilenko, ${ }^{108}$ A. Gavrilyuk, ${ }^{109}$ C. Gay, ${ }^{172}$ G. Gaycken, ${ }^{24}$ E. N. Gazis, ${ }^{10}$ C. N. P. Gee, ${ }^{141}$ J. Geisen, ${ }^{51}$ M. Geisen, ${ }^{97}$ M. P. Geisler, ${ }^{59 a}$ K. Gellerstedt, ${ }^{43 a, 43 b}$ C. Gemme,${ }^{53 b}$

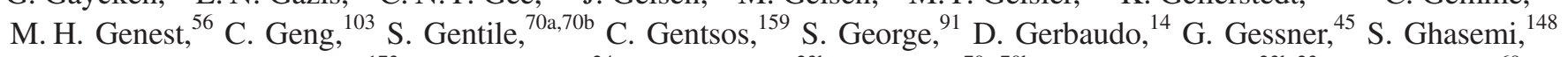
M. Ghasemi Bostanabad, ${ }^{173}$ M. Ghneimat, ${ }^{24}$ B. Giacobbe, ${ }^{23 b}$ S. Giagu, ${ }^{70 a, 70 b}$ N. Giangiacomi, ${ }^{23 b, 23 a}$ P. Giannetti, ${ }^{69 a}$ S. M. Gibson, ${ }^{91}$ M. Gignac, ${ }^{143}$ D. Gillberg, ${ }^{33}$ G. Gilles, ${ }^{179}$ D. M. Gingrich, ${ }^{3, e}$ M. P. Giordani, ${ }^{64 a, 64 c}$ F. M. Giorgi, ${ }^{23 b}$ P. F. Giraud,${ }^{142}$ P. Giromini ${ }^{57}$ G. Giugliarelli, ${ }^{64 a 64 c}$ D. Giugni, ${ }^{66 a}$ F. Giuli, ${ }^{131}$ M. Giulini, ${ }^{59 b}$ S. Gkaitatzis, ${ }^{159}$ I. Gkialas, ${ }^{9, t}$ E. L. Gkougkousis, ${ }^{14}$ P. Gkountoumis, ${ }^{10}$ L. K. Gladilin, ${ }^{111}$ C. Glasman, ${ }^{96}$ J. Glatzer, ${ }^{14}$ P. C. F. Glaysher, ${ }^{44}$ A. Glazov, ${ }^{44}$ M. Goblirsch-Kolb, ${ }^{26}$ J. Godlewski, ${ }^{82}$ S. Goldfarb, ${ }^{102}$ T. Golling, ${ }^{52}$ D. Golubkov, ${ }^{140}$ A. Gomes, ${ }^{16 a, 136 b, 136 \mathrm{~d}}$ R. Goncalves Gama, ${ }^{78 a}$ R. Gonçalo, ${ }^{136 a}$ G. Gonella, ${ }^{50}$ L. Gonella, ${ }^{21}$ A. Gongadze, ${ }^{77}$ F. Gonnella, ${ }^{21}$ J. L. Gonski, ${ }^{57}$ S. González de la Hoz, ${ }^{171}$ S. Gonzalez-Sevilla, ${ }^{52}$ L. Goossens,${ }^{35}$ P. A. Gorbounov, ${ }^{109}$ H. A. Gordon, ${ }^{29}$ B. Gorini, ${ }^{35}$ E. Gorini, ${ }^{65 a, 65 b}$ A. Gorišek, ${ }^{89}$ A. T. Goshaw, ${ }^{47}$ C. Gössling, ${ }^{45}$ M. I. Gostkin,${ }^{77}$ C. A. Gottardo, ${ }^{24}$ C. R. Goudet, ${ }^{128}$ D. Goujdami, ${ }^{34 c}$ A. G. Goussiou, ${ }^{145}$ N. Govender, ${ }^{32 b, u}$ C. Goy, ${ }^{5}$ E. Gozani, ${ }^{157}$ I. Grabowska-Bold, ${ }^{81 a}$ P. O. J. Gradin, ${ }^{169}$ E. C. Graham, ${ }^{88}$ J. Gramling, ${ }^{168}$ E. Gramstad, ${ }^{130}$ S. Grancagnolo, ${ }^{19}$ V. Gratchev,${ }^{134}$ P. M. Gravila,,${ }^{27 f}$ C. Gray, ${ }^{55}$ H. M. Gray,${ }^{18}$ Z. D. Greenwood, ${ }^{93, v}$ C. Grefe, ${ }^{24}$ K. Gregersen, ${ }^{92}$ I. M. Gregor, ${ }^{44}$ P. Grenier, ${ }^{150}$ K. Grevtsov, ${ }^{44}$ J. Griffiths, ${ }^{8}$ A. A. Grillo, ${ }^{143}$ K. Grimm, ${ }^{150}$ S. Grinstein, ${ }^{14, w}$ Ph. Gris, ${ }^{37}$ J.-F. Grivaz, ${ }^{128}$ S. Groh, ${ }^{97}$ E. Gross, ${ }^{177}$ J. Grosse-Knetter, ${ }^{51}$ G. C. Grossi,${ }^{93}$ Z. J. Grout ${ }^{92}$ C. Grud, ${ }^{103}$ A. Grummer, ${ }^{116}$ L. Guan, ${ }^{103}$ W. Guan, ${ }^{178}$ J. Guenther, ${ }^{35}$ A. Guerguichon, ${ }^{128}$ F. Guescini, ${ }^{165 a}$ D. Guest, ${ }^{168}$ R. Gugel, ${ }^{50}$ B. Gui, ${ }^{122}$ T. Guillemin,,${ }^{5}$ S. Guindon, ${ }^{35}$ U. Gul, ${ }^{55}$ C. Gumpert, ${ }^{35}$ J. Guo, ${ }^{58 c}$ W. Guo, ${ }^{103}$ Y. Guo, ${ }^{58 a, x}$ Z. Guo, ${ }^{99}$ R. Gupta, ${ }^{41}$ S. Gurbuz, ${ }^{12 \mathrm{c}}$ G. Gustavino, ${ }^{124}$ B. J. Gutelman, ${ }^{157}$ P. Gutierrez, ${ }^{124}$ C. Gutschow, ${ }^{92}$ C. Guyot, ${ }^{142}$ M. P. Guzik, ${ }^{81 a}$ C. Gwenlan, ${ }^{131}$ C. B. Gwilliam, ${ }^{88}$ A. Haas, ${ }^{121}$ C. Haber, ${ }^{18}$ H. K. Hadavand,${ }^{8}$ N. Haddad,${ }^{34 \mathrm{e}}$ A. Hadef, ${ }^{58 \mathrm{a}}$ S. Hageböck, ${ }^{24}$ M. Hagihara, ${ }^{166}$ H. Hakobyan, ${ }^{181, a}$ M. Haleem, ${ }^{174}$ J. Haley, ${ }^{125}$ G. Halladjian, ${ }^{104}$ G. D. Hallewell, ${ }^{99}$ K. Hamacher, ${ }^{179}$ P. Hamal, ${ }^{126}$ K. Hamano, ${ }^{173}$ A. Hamilton, ${ }^{32 a}$ G. N. Hamity, ${ }^{146}$ K. Han ${ }^{58 a, y}$ L. Han,${ }^{58 a}$ S. Han, ${ }^{15 d}$ K. Hanagaki, ${ }^{79, z}$ M. Hance, ${ }^{143}$ D. M. Handl, ${ }^{112}$ B. Haney, ${ }^{133}$ R. Hankache,${ }^{132}$ P. Hanke ${ }^{59 a}$ E. Hansen,${ }^{94}$ J. B. Hansen, ${ }^{39}$ J. D. Hansen, ${ }^{39}$ M. C. Hansen, ${ }^{24}$ P. H. Hansen, ${ }^{39}$ K. Hara, ${ }^{166}$ A. S. Hard,${ }^{178}$ T. Harenberg, ${ }^{179}$ S. Harkusha, ${ }^{105}$ P. F. Harrison, ${ }^{175}$ N. M. Hartmann, ${ }^{112}$ Y. Hasegawa, ${ }^{147}$ A. Hasib ${ }^{48}$ S. Hassani, ${ }^{142}$ S. Haug, ${ }^{20}$ R. Hauser, ${ }^{104}$ L. Hauswald, ${ }^{46}$ L. B. Havener, ${ }^{38}$

M. Havranek, ${ }^{138}$ C. M. Hawkes, ${ }^{21}$ R. J. Hawkings,${ }^{35}$ D. Hayden, ${ }^{104}$ C. Hayes, ${ }^{152}$ C. P. Hays,${ }^{131}$ J. M. Hays, ${ }^{90}$

H. S. Hayward, ${ }^{88}$ S. J. Haywood, ${ }^{141}$ M. P. Heath, ${ }^{48}$ V. Hedberg, ${ }^{94}$ L. Heelan, ${ }^{8}$ S. Heer, ${ }^{24}$ K. K. Heidegger, ${ }^{50}$ J. Heilman ${ }^{33}$ S. Heim, ${ }^{44}$ T. Heim, ${ }^{18}$ B. Heinemann, ${ }^{44, a a}$ J. J. Heinrich, ${ }^{12}$ L. Heinrich, ${ }^{121}$ C. Heinz,${ }^{54}$ J. Hejbal, ${ }^{137}$ L. Helary, ${ }^{35}$ A. Held, ${ }^{172}$ S. Hellesund, ${ }^{130}$ S. Hellman, ${ }^{43 a, 43 b}$ C. Helsens,${ }^{35}$ R. C. W. Henderson, ${ }^{87}$ Y. Heng, ${ }^{178}$ S. Henkelmann, ${ }^{172}$

A. M. Henriques Correia, ${ }^{35}$ G. H. Herbert, ${ }^{19}$ H. Herde, ${ }^{26}$ V. Herget, ${ }^{174}$ Y. Hernández Jiménez,${ }^{32 c}$ H. Herr, ${ }^{97}$ G. Herten,${ }^{50}$ R. Hertenberger, ${ }^{112}$ L. Hervas, ${ }^{35}$ T. C. Herwig, ${ }^{133}$ G. G. Hesketh, ${ }^{92}$ N. P. Hessey, ${ }^{165}$ J. W. Hetherly, ${ }^{41}$ S. Higashino, ${ }^{79}$ 
E. Higón-Rodriguez, ${ }^{171}$ K. Hildebrand ${ }^{36}$ E. Hill, ${ }^{173}$ J. C. Hill, ${ }^{31}$ K. K. Hill, ${ }^{29}$ K. H. Hiller ${ }^{44}$ S. J. Hillier, ${ }^{21}$ M. Hils ${ }^{46}$ I. Hinchliffe, ${ }^{18}$ M. Hirose, ${ }^{129}$ D. Hirschbuehl, ${ }^{179}$ B. Hiti, ${ }^{89}$ O. Hladik, ${ }^{137}$ D. R. Hlaluku, ${ }^{32 c}$ X. Hoad, ${ }^{48}$ J. Hobbs, ${ }^{152}$ N. Hod, ${ }^{165 a}$ M. C. Hodgkinson, ${ }^{146}$ A. Hoecker, ${ }^{35}$ M. R. Hoeferkamp, ${ }^{116}$ F. Hoenig, ${ }^{112}$ D. Hohn, ${ }^{24}$ D. Hohov, ${ }^{128}$ T. R. Holmes, ${ }^{36}$ M. Holzbock, ${ }^{112}$ M. Homann, ${ }^{45}$ S. Honda, ${ }^{166}$ T. Honda, ${ }^{79}$ T. M. Hong, ${ }^{135}$ A. Hönle, ${ }^{113}$ B. H. Hooberman, ${ }^{170}$ W. H. Hopkins, ${ }^{127}$ Y. Horii, ${ }^{115}$ P. Horn, ${ }^{46}$ A. J. Horton, ${ }^{149}$ L. A. Horyn, ${ }^{36}$ J-Y. Hostachy, ${ }^{56}$ A. Hostiuc, ${ }^{145}$ S. Hou, ${ }^{155}$

A. Hoummada, ${ }^{34 a}$ J. Howarth, ${ }^{98}$ J. Hoya ${ }^{86}$ M. Hrabovsky, ${ }^{126}$ J. Hrdinka,${ }^{35}$ I. Hristova, ${ }^{19}$ J. Hrivnac, ${ }^{128}$ A. Hrynevich, ${ }^{106}$

T. Hryn'ova, ${ }^{5}$ P. J. Hsu, ${ }^{62}$ S.-C. Hsu, ${ }^{145}$ Q. Hu, ${ }^{29}$ S. Hu,${ }^{58 c}$ Y. Huang, ${ }^{15 a}$ Z. Hubacek, ${ }^{138}$ F. Hubaut ${ }^{99}$ M. Huebner, ${ }^{24}$ F. Huegging, ${ }^{24}$ T. B. Huffman, ${ }^{131}$ E. W. Hughes,${ }^{38}$ M. Huhtinen,,${ }^{35}$ R. F. H. Hunter,${ }^{33}$ P. Huo, ${ }^{152}$ A. M. Hupe, ${ }^{33}$

N. Huseynov, ${ }^{77, d}$ J. Huston, ${ }^{104}$ J. Huth,${ }^{57}$ R. Hyneman, ${ }^{103}$ G. Iacobucci, ${ }^{52}$ G. Iakovidis, ${ }^{29}$ I. Ibragimov, ${ }^{148}$ L. Iconomidou-Fayard, ${ }^{128}$ Z. Idrissi, ${ }^{34 \mathrm{e}}$ P. Iengo, ${ }^{35}$ R. Ignazzi, ${ }^{39}$ O. Igonkina, ${ }^{118, \text { bb }}$ R. Iguchi, ${ }^{160}{ }^{\text {T. Iizawa }}{ }^{52}$ Y. Ikegami, ${ }^{79}$ M. Ikeno, ${ }^{79}$ D. Iliadis, ${ }^{159} \mathrm{~N}$. Ilic, ${ }^{150} \mathrm{~F}$. Iltzsche, ${ }^{46} \mathrm{G}$. Introzzi, ${ }^{68 \mathrm{a}, 68 \mathrm{~b}} \mathrm{M}$. Iodice, ${ }^{72 \mathrm{a}} \mathrm{K}$. Iordanidou, ${ }^{38} \mathrm{~V}$. Ippolito, ${ }^{70 \mathrm{a}, 70 \mathrm{~b}}$ M. F. Isacson, ${ }^{169} \mathrm{~N}$. Ishijima, ${ }^{129} \mathrm{M}$. Ishino, ${ }^{160} \mathrm{M}$. Ishitsuka, ${ }^{162} \mathrm{C}$. Issever, ${ }^{131} \mathrm{~S}$. Istin ${ }^{12 \mathrm{ccc}, \mathrm{F}}$. Ito, ${ }^{166} \mathrm{~J}$. M. Iturbe Ponce, ${ }^{61 \mathrm{a}}$ R. Iuppa ${ }^{73 a, 73 b}$ A. Ivina, ${ }^{177}$ H. Iwasaki, ${ }^{79}$ J. M. Izen, ${ }^{42}$ V. Izzo, ${ }^{67 a}$ S. Jabbar, ${ }^{3}$ P. Jacka, ${ }^{137}$ P. Jackson, ${ }^{1}$ R. M. Jacobs, ${ }^{24}$ V. Jain, ${ }^{2}$ G. Jäkel, ${ }^{179}$ K. B. Jakobi,${ }^{97}$ K. Jakobs,${ }^{50}$ S. Jakobsen, ${ }^{74}$ T. Jakoubek, ${ }^{137}$ D. O. Jamin, ${ }^{125}$ D. K. Jana, ${ }^{93}$ R. Jansky, ${ }^{52}$ J. Janssen, ${ }^{24}$ M. Janus,${ }^{51}$ P. A. Janus, ${ }^{81 a}$ G. Jarlskog, ${ }^{94}$ N. Javadov, ${ }^{77, d}$ T. Javůrek, ${ }^{50}$ M. Javurkova, ${ }^{50}$ F. Jeanneau, ${ }^{142}$ L. Jeanty, ${ }^{18}$ J. Jejelava, ${ }^{156 a, d d}$ A. Jelinskas, ${ }^{175}$ P. Jenni, ${ }^{50, \text { ee }}$ J. Jeong, ${ }^{44}$ C. Jeske,${ }^{175}$ S. Jézéquel, ${ }^{5}$ H. Ji, ${ }^{178}$ J. Jia, ${ }^{152}$ H. Jiang, ${ }^{76}$ Y. Jiang, ${ }^{58 \mathrm{a}}$ Z. Jiang, ${ }^{150}$ S. Jiggins, ${ }^{50}$ F. A. Jimenez Morales, ${ }^{37}$ J. Jimenez Pena, ${ }^{171}$ S. Jin, ${ }^{15 \mathrm{c}}$ A. Jinaru, ${ }^{27 \mathrm{~b}}$ O. Jinnouchi, ${ }^{162}$ H. Jivan, ${ }^{32 \mathrm{c}}$ P. Johansson, ${ }^{146}$ K. A. Johns, ${ }^{7}$ C. A. Johnson, ${ }^{63}$ W. J. Johnson, ${ }^{145}$ K. Jon-And, ${ }^{43 a, 43 b}$ R. W. L. Jones, ${ }^{87}$ S. D. Jones, ${ }^{153}$ S. Jones, ${ }^{7}$ T. J. Jones, ${ }^{88}$ J. Jongmanns, ${ }^{59 a}$ P. M. Jorge, ${ }^{136 a, 136 b}$ J. Jovicevic, ${ }^{165 a}$ X. Ju, ${ }^{178}$ J. J. Junggeburth, ${ }^{113}$ A. Juste Rozas, ${ }^{14, w}$ A. Kaczmarska, ${ }^{82}$ M. Kado, ${ }^{128}$ H. Kagan, ${ }^{122}$ M. Kagan, ${ }^{150}$ T. Kaji, ${ }^{176}$ E. Kajomovitz, ${ }^{157}$ C. W. Kalderon, ${ }^{94}$ A. Kaluza, ${ }^{97}$ S. Kama, ${ }^{41}$ A. Kamenshchikov, ${ }^{140}$ L. Kanjir, ${ }^{89}$ Y. Kano, ${ }^{160}$ V. A. Kantserov,${ }^{110}$ J. Kanzaki, ${ }^{79}$ B. Kaplan, ${ }^{121}$ L. S. Kaplan, ${ }^{178}$ D. Kar, ${ }^{32 \mathrm{c}}$ M. J. Kareem, ${ }^{165 \mathrm{~b}}$ E. Karentzos, ${ }^{10}$ S. N. Karpov, ${ }^{77}$ Z. M. Karpova, ${ }^{77}$ V. Kartvelishvili, ${ }^{87}$ A. N. Karyukhin, ${ }^{140}$ K. Kasahara, ${ }^{166}$ L. Kashif,${ }^{178}$ R. D. Kass, ${ }^{122}$ A. Kastanas, ${ }^{151}$ Y. Kataoka, ${ }^{160}$ C. Kato, ${ }^{160}$ J. Katzy, ${ }^{44}$ K. Kawade ${ }^{80}$ K. Kawagoe, ${ }^{85}$ T. Kawamoto, ${ }^{160}$ G. Kawamura, ${ }^{51}$ E. F. Kay, ${ }^{88}$ V. F. Kazanin, ${ }^{120 b, 120 a}$ R. Keeler, ${ }^{173}$ R. Kehoe, ${ }^{41}$ J. S. Keller, ${ }^{33}$ E. Kellermann, ${ }^{94}$ J. J. Kempster, ${ }^{21}$ J. Kendrick, ${ }^{21}$ O. Kepka, ${ }^{137}$ S. Kersten, ${ }^{179}$

B. P. Kerševan ${ }^{89}$ R. A. Keyes, ${ }^{101}$ M. Khader, ${ }^{170}$ F. Khalil-Zada, ${ }^{13}$ A. Khanov, ${ }^{125}$ A. G. Kharlamov, ${ }^{120 b, 120 a}$ T. Kharlamova, ${ }^{120 b, 120 \mathrm{a}}$ A. Khodinov, ${ }^{163}$ T. J. Khoo, ${ }^{52}$ E. Khramov, ${ }^{77}$ J. Khubua, ${ }^{156 b}$ S. Kido, ${ }^{80}$ M. Kiehn, ${ }^{52}$ C. R. Kilby, ${ }^{91}$ S. H. Kim, ${ }^{166}$ Y. K. Kim, ${ }^{36}$ N. Kimura, ${ }^{64,64 \mathrm{c}}$ O. M. Kind, ${ }^{19}$ B. T. King, ${ }^{88}$ D. Kirchmeier, ${ }^{46}$ J. Kirk, ${ }^{141}$ A. E. Kiryunin,${ }^{113}$ T. Kishimoto, ${ }^{160}$ D. Kisielewska, ${ }^{81 a}$ V. Kitali, ${ }^{44}$ O. Kivernyk, ${ }^{5}$ E. Kladiva, ${ }^{28 b}$ T. Klapdor-Kleingrothaus, ${ }^{50}$ M. H. Klein, ${ }^{103}$ M. Klein, ${ }^{88}$ U. Klein, ${ }^{88}$ K. Kleinknecht, ${ }^{97}$ P. Klimek, ${ }^{119}$ A. Klimentov, ${ }^{29}$ R. Klingenberg,,${ }^{45, a}$ T. Klingl, ${ }^{24}$

T. Klioutchnikova, ${ }^{35}$ F. F. Klitzner, ${ }^{112}$ P. Kluit, ${ }^{118}$ S. Kluth,${ }^{113}$ E. Kneringer, ${ }^{74}$ E. B. F. G. Knoops, ${ }^{99}$ A. Knue, ${ }^{50}$ A. Kobayashi, ${ }^{160}$ D. Kobayashi, ${ }^{85}$ T. Kobayashi, ${ }^{160}$ M. Kobel,${ }^{46}$ M. Kocian, ${ }^{150}$ P. Kodys, ${ }^{139}$ T. Koffas,${ }^{33}$ E. Koffeman, ${ }^{118}$ N. M. Köhler, ${ }^{113}$ T. Koi, ${ }^{150}$ M. Kolb, ${ }^{59 b}$ I. Koletsou, ${ }^{5}$ T. Kondo, ${ }^{79}$ N. Kondrashova ${ }^{58 c}$ K. Köneke, ${ }^{50}$ A. C. König, ${ }^{117}$ T. Kono, ${ }^{79}$ R. Konoplich, ${ }^{121, f f}$ V. Konstantinides, ${ }^{92}$ N. Konstantinidis, ${ }^{92}$ B. Konya,${ }^{94}$ R. Kopeliansky, ${ }^{63}$ S. Koperny, ${ }^{81 a}$ K. Korcyl, ${ }^{82}$ K. Kordas, ${ }^{159}$ A. Korn, ${ }^{92}$ I. Korolkov, ${ }^{14}$ E. V. Korolkova, ${ }^{146}$ O. Kortner, ${ }^{13}$ S. Kortner, ${ }^{13}$ T. Kosek, ${ }^{139}$ V. V. Kostyukhin, ${ }^{24}$ A. Kotwal, ${ }^{47}$ A. Koulouris, ${ }^{10}$ A. Kourkoumeli-Charalampidi, ${ }^{68 a, 68 b}$ C. Kourkoumelis, ${ }^{9}$ E. Kourlitis, ${ }^{146}$ V. Kouskoura, ${ }^{29}$ A. B. Kowalewska, ${ }^{82}$ R. Kowalewski, ${ }^{173}$ T. Z. Kowalski, ${ }^{81 a}$ C. Kozakai, ${ }^{160}$ W. Kozanecki, ${ }^{142}$ A. S. Kozhin, ${ }^{140}$ V. A. Kramarenko, ${ }^{111}$ G. Kramberger,${ }^{89}$ D. Krasnopevtsev, ${ }^{110}$ M. W. Krasny, ${ }^{132}$ A. Krasznahorkay, ${ }^{35}$ D. Krauss, ${ }^{113}$ J. A. Kremer, ${ }^{81 a}$ J. Kretzschmar, ${ }^{88}$ P. Krieger, ${ }^{164}$ K. Krizka, ${ }^{18}$ K. Kroeninger, ${ }^{45}$ H. Kroha, ${ }^{113}$ J. Kroll, ${ }^{137}$ J. Kroll, ${ }^{133}$ J. Krstic, ${ }^{16}$ U. Kruchonak,${ }^{77}$ H. Krüger, ${ }^{24}$ N. Krumnack, ${ }^{76}$ M. C. Kruse, ${ }^{47}$ T. Kubota, ${ }^{102}$ S. Kuday, ${ }^{4 b}$ J. T. Kuechler, ${ }^{179}$ S. Kuehn, ${ }^{35}$ A. Kugel, ${ }^{59 a}$ F. Kuger, ${ }^{174}$ T. Kuhll, ${ }^{44}$ V. Kukhtin, ${ }^{77}$ R. Kukla, ${ }^{99}$ Y. Kulchitsky, ${ }^{105}$ S. Kuleshov, ${ }^{14 \mathrm{~b}}$ Y. P. Kulinich, ${ }^{170}$ M. Kuna, ${ }^{56}$ T. Kunigo, ${ }^{83}$ A. Kupco, ${ }^{137}$ T. Kupfer, ${ }^{45}$ O. Kuprash, ${ }^{158}$ H. Kurashige ${ }^{80}$ L. L. Kurchaninov, ${ }^{165 a}$ Y. A. Kurochkin, ${ }^{105}$ M. G. Kurth, ${ }^{15 d}$ E. S. Kuwertz, ${ }^{173}$ M. Kuze, ${ }^{162}$ J. Kvita, ${ }^{126}$ T. Kwan, ${ }^{101}$ A. La Rosa ${ }^{113}$ J. L. La Rosa Navarro, ${ }^{78 d}$ L. La Rotonda, ${ }^{40 b, 40 a}$ F. La Ruffa, ${ }^{40 b, 40 a}$ C. Lacasta, ${ }^{171}$ F. Lacava, ${ }^{70 a}$,70b J. Lacey, ${ }^{44}$ D. P. J. Lack, ${ }^{98}$ H. Lacker, ${ }^{19}$ D. Lacour, ${ }^{132}$ E. Ladygin, ${ }^{77}$ R. Lafaye, ${ }^{5}$ B. Laforge, ${ }^{132}$ T. Lagouri, ${ }^{32 c}$ S. Lai, ${ }^{51}$ S. Lammers,${ }^{63}$ W. Lampl, ${ }^{7}$ E. Lançon, ${ }^{29}$ U. Landgraf,${ }^{50}$ M. P. J. Landon, ${ }^{90}$ M. C. Lanfermann, ${ }^{52}$ V. S. Lang, ${ }^{44}$ J. C. Lange, ${ }^{14}$ R. J. Langenberg, ${ }^{35}$ A. J. Lankford, ${ }^{168}$ F. Lanni, ${ }^{29}$ K. Lantzsch, ${ }^{24}$ A. Lanza, ${ }^{68 a}$ A. Lapertosa, ${ }^{53 b, 53 a}$ S. Laplace, ${ }^{132}$ J. F. Laporte, ${ }^{142}$ T. Lari, ${ }^{66 a}$ F. Lasagni Manghi, ${ }^{23 b, 23 a}$ M. Lassnig, ${ }^{35}$ T. S. Lau, ${ }^{61 a}$ A. Laudrain, ${ }^{128}$ A. T. Law, ${ }^{143}$ P. Laycock, ${ }^{88}$ M. Lazzaroni, ${ }^{66 a, 66 b}$ B. Le, ${ }^{102}$ O. Le Dortz, ${ }^{132}$ E. Le Guirriec, ${ }^{99}$ E. P. Le Quilleuc, ${ }^{142}$ M. LeBlanc, ${ }^{7}$ T. LeCompte, ${ }^{6}$ 
F. Ledroit-Guillon, ${ }^{56}$ C. A. Lee, ${ }^{29}$ G. R. Lee, ${ }^{144 a}$ L. Lee,${ }^{57}$ S. C. Lee ${ }^{155}$ B. Lefebvre, ${ }^{101}$ M. Lefebvre, ${ }^{173}$ F. Legger, ${ }^{112}$ C. Leggett, ${ }^{18}$ N. Lehmann, ${ }^{179}$ G. Lehmann Miotto, ${ }^{35}$ W. A. Leight, ${ }^{44}$ A. Leisos, ${ }^{159, g g}$ M. A. L. Leite, ${ }^{78 d}$ R. Leitner, ${ }^{139}$ D. Lellouch, ${ }^{177}$ B. Lemmer, ${ }^{51}$ K. J. C. Leney, ${ }^{92}$ T. Lenz, ${ }^{24}$ B. Lenzi, ${ }^{35}$ R. Leone, ${ }^{7}$ S. Leone, ${ }^{69 a}$ C. Leonidopoulos,${ }^{48}$ G. Lerner, ${ }^{153}$ C. Leroy, ${ }^{107}$ R. Les, ${ }^{164}$ A. A. J. Lesage, ${ }^{142}$ C. G. Lester, ${ }^{31}$ M. Levchenko, ${ }^{134}$ J. Levêque, ${ }^{5}$ D. Levin, ${ }^{103}$ L. J. Levinson, ${ }^{177}$ D. Lewis, ${ }^{90}$ B. Li ${ }^{103}$ C-Q. Li ${ }^{58 \mathrm{a}} \mathrm{H} . \mathrm{Li},{ }^{58 \mathrm{~b}} \mathrm{~L} . \mathrm{Li},{ }^{58 \mathrm{c}} \mathrm{Q} . \mathrm{Li},{ }^{15 \mathrm{~d}} \mathrm{Q} . \mathrm{Y} . \mathrm{Li}^{58 \mathrm{a}} \mathrm{S}^{\mathrm{Li}},{ }^{58 \mathrm{~d}, 58 \mathrm{c}} \mathrm{X} . \mathrm{Li},{ }^{58 \mathrm{c}} \mathrm{Y} . \mathrm{Li}^{1}{ }^{148}$ Z. Liang, ${ }^{15 a}$ B. Liberti, ${ }^{71 a}$ A. Liblong, ${ }^{164}$ K. Lie, ${ }^{61 \mathrm{c}}$ S. Liem, ${ }^{118}$ A. Limosani, ${ }^{154}$ C. Y. Lin, ${ }^{31}$ K. Lin,${ }^{104}$ T. H. Lin, ${ }^{97}$ R. A. Linck, ${ }^{63}$ B. E. Lindquist, ${ }^{152}$ A. L. Lionti, ${ }^{52}$ E. Lipeles, ${ }^{133}$ A. Lipniacka,,${ }^{17}$ M. Lisovyi, ${ }^{59 b}$ T. M. Liss, ${ }^{170, \text { hh }}$ A. Lister, ${ }^{172}$ A. M. Litke, ${ }^{143}$ J. D. Little, ${ }^{8}$ B. Liu, ${ }^{76}$ B. L Liu, ${ }^{6}$ H. B. Liu, ${ }^{29}$ H. Liu, ${ }^{103}$ J. B. Liu, ${ }^{58 a}$ J. K. K. Liu, ${ }^{131}$ K. Liu, ${ }^{132}$ M. Liu, ${ }^{58 a}$ P. Liu, ${ }^{18}$ Y. Liu, ${ }^{15 a}$ Y. L. Liu, ${ }^{58 \mathrm{a}}$ Y. W. Liu, ${ }^{58 \mathrm{a}}$ M. Livan, ${ }^{68 a, 68 \mathrm{~b}}$ A. Lleres, ${ }^{56}$ J. Llorente Merino, ${ }^{15 \mathrm{a}}$ S. L. Lloyd, ${ }^{90}$ C. Y. Lo, ${ }^{61 b}$ F. Lo Sterzo, ${ }^{41}$ E. M. Lobodzinska, ${ }^{44}$ P. Loch,${ }^{7}$ F. K. Loebinger, ${ }^{98}$ A. Loesle, ${ }^{50}$ K. M. Loew, ${ }^{26}$ T. Lohse, ${ }^{19}$ K. Lohwasser, ${ }^{146}$ M. Lokajicek, ${ }^{137}$ B. A. Long, ${ }^{25}$ J. D. Long, ${ }^{170}$ R. E. Long, ${ }^{87}$ L. Longo, ${ }^{65 a, 65 b}$ K. A. Looper, ${ }^{122}$ J. A. Lopez, ${ }^{144 b}$ I. Lopez Paz, ${ }^{14}$ A. Lopez Solis, ${ }^{146}$ J. Lorenz, ${ }^{112}$ N. Lorenzo Martinez, ${ }^{5}$ M. Losada, ${ }^{22}$ P. J. Lösel, ${ }^{112}$ X. Lou, ${ }^{44}$ X. Lou, ${ }^{15 a}$ A. Lounis, ${ }^{128}$ J. Love, ${ }^{6}$ P. A. Love, ${ }^{87}$ J. J. Lozano Bahilo, ${ }^{171}$ H. Lu, ${ }^{61 a}$ M. Lu, ${ }^{58 a}$ N. Lu, ${ }^{103}$ Y. J. Lu, ${ }^{62}$ H. J. Lubatti, ${ }^{145}$ C. Luci, ${ }^{70 a, 70 b}$ A. Lucotte ${ }^{56}$ C. Luedtke ${ }^{50}$ F. Luehring, ${ }^{63}$ I. Luise, ${ }^{132}$ W. Lukas, ${ }^{74}$ L. Luminari, ${ }^{70 a}$ B. Lund-Jensen, ${ }^{151}$ M. S. Lutz, ${ }^{100}$ P. M. Luzi, ${ }^{132}$ D. Lynn, ${ }^{29}$ R. Lysak, ${ }^{177}$ E. Lytken, ${ }^{94}$ F. Lyu, ${ }^{15 a}$ V. Lyubushkin, ${ }^{77}$ H. Ma, ${ }^{29}$ L. L. Ma, ${ }^{58 b}$ Y. Ma, ${ }^{58 b}$ G. Maccarrone, ${ }^{49}$ A. Macchiolo, ${ }^{113}$ C. M. Macdonald, ${ }^{146}$ J. Machado Miguens, ${ }^{133,136 \mathrm{~b}}$ D. Madaffari, ${ }^{171}$ R. Madar, ${ }^{37}$ W. F. Mader ${ }^{46}$ A. Madsen, ${ }^{44}$ N. Madysa, ${ }^{46}$ J. Maeda ${ }^{80}$ K. Maekawa, ${ }^{160}$ S. Maeland, ${ }^{17}$ T. Maeno, ${ }^{29}$ A. S. Maevskiy, ${ }^{111}$ V. Magerl, ${ }^{50}$ C. Maidantchik ${ }^{78 b}$ T. Maier, ${ }^{112}$ A. Maio, ${ }^{136 a, 136 b, 136 \mathrm{~d}}$ O. Majersky, ${ }^{28 \mathrm{a}}$ S. Majewski, ${ }^{127}$ Y. Makida, ${ }^{79}$ N. Makovec, ${ }^{128}$ B. Malaescu, ${ }^{132}$ Pa. Malecki, ${ }^{82}$ V. P. Maleev, ${ }^{134}$ F. Malek, ${ }^{56}$ U. Mallik, ${ }^{75}$ D. Malon, ${ }^{6}$ C. Malone,${ }^{31}$ S. Maltezos, ${ }^{10}$ S. Malyukov,${ }^{35}$ J. Mamuzic, ${ }^{171}$ G. Mancini, ${ }^{49}$ I. Mandic,${ }^{89}$ J. Maneira, ${ }^{136 a}$ L. Manhaes de Andrade Filho, ${ }^{78 a}$ J. Manjarres Ramos, ${ }^{46}$ K. H. Mankinen, ${ }^{94}$ A. Mann, ${ }^{112}$ A. Manousos, ${ }^{74}$ B. Mansoulie, ${ }^{142}$ J. D. Mansour, ${ }^{15 a}$ M. Mantoani, ${ }^{51}$ S. Manzoni, ${ }^{66 a, 66 b}$ G. Marceca, ${ }^{30}$ L. March, ${ }^{52}$ L. Marchese, ${ }^{131}$ G. Marchiori, ${ }^{132}$ M. Marcisovsky, ${ }^{137}$ C. A. Marin Tobon, ${ }^{35}$

M. Marjanovic, ${ }^{37}$ D. E. Marley, ${ }^{103}$ F. Marroquim, ${ }^{78 b}$ Z. Marshall, ${ }^{18}$ M. U. F Martensson, ${ }^{169}$ S. Marti-Garcia, ${ }^{171}$

C. B. Martin, ${ }^{122}$ T. A. Martin, ${ }^{175}$ V. J. Martin, ${ }^{48}$ B. Martin dit Latour, ${ }^{17}$ M. Martinez, ${ }^{14, w}$ V. I. Martinez Outschoorn, ${ }^{100}$ S. Martin-Haugh, ${ }^{141}$ V. S. Martoiu, ${ }^{27 b}$ A. C. Martyniuk, ${ }^{92}$ A. Marzin, ${ }^{35}$ L. Masetti, ${ }^{97}$ T. Mashimo, ${ }^{160}$ R. Mashinistov, ${ }^{108}$ J. Masik, ${ }^{98}$ A. L. Maslennikov, ${ }^{120 b, 120 \mathrm{a}}$ L. H. Mason, ${ }^{102}$ L. Massa, ${ }^{71,71 \mathrm{~b}}$ P. Mastrandrea, ${ }^{5}$ A. Mastroberardino, ${ }^{40 \mathrm{~b}, 40 \mathrm{a}}$ T. Masubuchi, ${ }^{160}$ P. Mättig, ${ }^{179}$ J. Maurer ${ }^{27 b}$ B. Maček ${ }^{89}$ S. J. Maxfield, ${ }^{88}$ D. A. Maximov,${ }^{120 b, 120 a}$ R. Mazini,${ }^{155}$ I. Maznas, ${ }^{159}$ S. M. Mazza, ${ }^{143}$ N. C. Mc Fadden, ${ }^{116}$ G. Mc Goldrick, ${ }^{164}$ S. P. Mc Kee, ${ }^{103}$ A. McCarn, ${ }^{103}$ T. G. McCarthy, ${ }^{113}$ L. I. McClymont, ${ }^{92}$ E. F. McDonald, ${ }^{102}$ J. A. Mcfayden, ${ }^{35}$ G. Mchedlidze, ${ }^{51}$ M. A. McKay, ${ }^{41}$ K. D. McLean, ${ }^{173}$ S. J. McMahon, ${ }^{141}$ P. C. McNamara, ${ }^{102}$ C. J. McNicol, ${ }^{175}$ R. A. McPherson, ${ }^{173, n}$ J. E. Mdhluli, ${ }^{32 c}$ Z. A. Meadows, ${ }^{100}$ S. Meehan, ${ }^{145}$ T. Megy, ${ }^{50}$ S. Mehlhase, ${ }^{112}$ A. Mehta ${ }^{88}$ T. Meideck, ${ }^{56}$ B. Meirose, ${ }^{42}$ D. Melini, ${ }^{171, i i}$ B. R. Mellado Garcia,${ }^{32 c}$ J. D. Mellenthin, ${ }^{51}$ M. Melo, ${ }^{28 a}$ F. Meloni, ${ }^{20}$ A. Melzer, ${ }^{24}$ S. B. Menary, ${ }^{98}$ E. D. Mendes Gouveia, ${ }^{136 a}$ L. Meng, ${ }^{88}$ X. T. Meng, ${ }^{103}$ A. Mengarelli, ${ }^{23 b, 23 a}$ S. Menke, ${ }^{113}$ E. Meoni, ${ }^{40 b, 40 a}$ S. Mergelmeyer, ${ }^{19}$ C. Merlassino, ${ }^{20}$ P. Mermod, ${ }^{52}$ L. Merola, ${ }^{67 a, 67 b}$ C. Meroni, ${ }^{66 a}$ F. S. Merritt, ${ }^{36}$ A. Messina, ${ }^{70 a, 70 b}$ J. Metcalfe, ${ }^{6}$ A. S. Mete, ${ }^{168}$ C. Meyer, ${ }^{133}$ J. Meyer, ${ }^{157}$ J-P. Meyer, ${ }^{142}$ H. Meyer Zu Theenhausen, ${ }^{59 a}$ F. Miano, ${ }^{153}$ R. P. Middleton, ${ }^{141}$ L. Mijović, ${ }^{48}$ G. Mikenberg, ${ }^{177}$ M. Mikestikova, ${ }^{137}$ M. Mikuž,,${ }^{89}$ M. Milesi, ${ }^{102}$ A. Milic, ${ }^{164}$ D. A. Millar, ${ }^{90}$ D. W. Miller, ${ }^{36}$ A. Milov, ${ }^{177}$ D. A. Milstead,${ }^{43 a, 43 b}$ A. A. Minaenko, ${ }^{140}$ M. Miñano Moya, ${ }^{171}$ I. A. Minashvili, ${ }^{156 b}$ A. I. Mincer, ${ }^{121}$ B. Mindur,${ }^{81 a}$ M. Mineev,${ }^{77}$ Y. Minegishi, ${ }^{160}$ Y. Ming, ${ }^{178}$ L. M. Mir, ${ }^{14}$ A. Mirto, ${ }^{65 a, 65 b}$ K. P. Mistry, ${ }^{133}$ T. Mitani, ${ }^{176}$ J. Mitrevski, ${ }^{112}$ V. A. Mitsou, ${ }^{171}$ A. Miucci,${ }^{20}$ P. S. Miyagawa ${ }^{146}$ A. Mizukami ${ }^{79}$ J. U. Mjörnmark,${ }^{94}$ T. Mkrtchyan, ${ }^{181}$ M. Mlynarikova, ${ }^{139}$ T. Moa, ${ }^{43 a, 43 b}$ K. Mochizuki, ${ }^{107}$ P. Mogg, ${ }^{50}$ S. Mohapatra, ${ }^{38}$ S. Molander, ${ }^{43 a}, 43 \mathrm{~b}$ R. Moles-Valls, ${ }^{24}$ M. C. Mondragon, ${ }^{104}$ K. Mönig, ${ }^{44}$ J. Monk, ${ }^{39}$ E. Monnier, ${ }^{99}$ A. Montalbano, ${ }^{149}$ J. Montejo Berlingen, ${ }^{35}$ F. Monticelli, ${ }^{86}$ S. Monzani ${ }^{66 a}$ R. W. Moore, ${ }^{3}$ N. Morange, ${ }^{128}$ D. Moreno, ${ }^{22}$ M. Moreno Llácer, ${ }^{35}$ P. Morettini, ${ }^{53 b}$ M. Morgenstern, ${ }^{118}$ S. Morgenstern, ${ }^{35}$ D. Mori, ${ }^{149}$ T. Mori, ${ }^{160}$ M. Morii, ${ }^{57}$ M. Morinaga, ${ }^{176}$ V. Morisbak, ${ }^{130}$ A. K. Morley, ${ }^{35}$ G. Mornacchi, ${ }^{35}$ A. P. Morris ${ }^{92}$ J. D. Morris, ${ }^{90}$ L. Morvaj, ${ }^{152}$ P. Moschovakos, ${ }^{10}$ M. Mosidze, ${ }^{156 b}$ H. J. Moss, ${ }^{146}$ J. Moss, ${ }^{150, j j}$ K. Motohashi, ${ }^{162}$ R. Mount ${ }^{150}$ E. Mountricha, ${ }^{35}$ E. J. W. Moyse, ${ }^{100}$ S. Muanza, ${ }^{99}$ F. Mueller,${ }^{113}$ J. Mueller, ${ }^{135}$ R. S. P. Mueller, ${ }^{112}$ D. Muenstermann, ${ }^{87}$ P. Mullen, ${ }^{55}$ G. A. Mullier, ${ }^{20}$ F. J. Munoz Sanchez, ${ }^{98}$ P. Murin,${ }^{28 b}$ W. J. Murray, ${ }^{175,141}$ A. Murrone,${ }^{66,66 b}$ M. Muškinja, ${ }^{89}$ C. Mwewa, ${ }^{32 a}$ A. G. Myagkov, ${ }^{140, k \mathrm{kk}}$ J. Myers, ${ }^{127}$ M. Myska, ${ }^{138}$ B. P. Nachman, ${ }^{18}$ O. Nackenhorst ${ }^{45}$ K. Nagai, ${ }^{131}$ K. Nagano, ${ }^{79}$ Y. Nagasaka, ${ }^{60}$ K. Nagata, ${ }^{166}$ M. Nagel,${ }^{50}$ E. Nagy, ${ }^{99}$ A. M. Nairz, ${ }^{35}$ Y. Nakahama, ${ }^{115}$ K. Nakamura, ${ }^{79}$ T. Nakamura, ${ }^{160}$ I. Nakano, ${ }^{123}$ H. Nanjo, ${ }^{129}$ F. Napolitano, ${ }^{59 a}$ R. F. Naranjo Garcia, ${ }^{44}$ R. Narayan, ${ }^{11}$ D. I. Narrias Villar, ${ }^{59 a}$ I. Naryshkin, ${ }^{134}$ 
T. Naumann, ${ }^{44}$ G. Navarro, ${ }^{22}$ R. Nayyar, ${ }^{7}$ H. A. Neal, ${ }^{103}$ P. Y. Nechaeva, ${ }^{108}$ T. J. Neep, ${ }^{142}$ A. Negri, ${ }^{68 a, 68 b}$ M. Negrini, ${ }^{23 b}$ S. Nektarijevic, ${ }^{117}$ C. Nellist, ${ }^{51}$ M.E. Nelson, ${ }^{131}$ S. Nemecek, ${ }^{137}$ P. Nemethy, ${ }^{121}$ M. Nessi, ${ }^{35,11}$ M. S. Neubauer, ${ }^{170}$ M. Neumann, ${ }^{179}$ P. R. Newman, ${ }^{21}$ T. Y. Ng, ${ }^{61 \mathrm{c}}$ Y. S. Ng, ${ }^{19}$ H. D. N. Nguyen, ${ }^{99}$ T. Nguyen Manh,${ }^{107}$ E. Nibigira, ${ }^{37}$ R. B. Nickerson, ${ }^{131}$ R. Nicolaidou, ${ }^{142}$ J. Nielsen, ${ }^{143}$ N. Nikiforou, ${ }^{11}$ V. Nikolaenko, ${ }^{140, k k}$ I. Nikolic-Audit, ${ }^{132}$ K. Nikolopoulos, ${ }^{21}$ P. Nilsson, ${ }^{29}$ Y. Ninomiya,${ }^{79}$ A. Nisati, ${ }^{70 a}$ N. Nishu, ${ }^{58 c}$ R. Nisius, ${ }^{113}$ I. Nitsche,${ }^{45}$ T. Nitta, ${ }^{176}$ T. Nobe,${ }^{160}$ Y. Noguchi, ${ }^{83}$ M. Nomachi, ${ }^{129}$ I. Nomidis, ${ }^{132}$ M. A. Nomura, ${ }^{29}$ T. Nooney,${ }^{90}$ M. Nordberg, ${ }^{35}$ N. Norjoharuddeen, ${ }^{131}$ T. Novak, ${ }^{89}$ O. Novgorodova, ${ }^{46}$ R. Novotny, ${ }^{138}$ M. Nozaki, ${ }^{79}$ L. Nozka,${ }^{126}$ K. Ntekas, ${ }^{168}$ E. Nurse, ${ }^{92}$ F. Nuti, ${ }^{102}$ F. G. Oakham, ${ }^{33, e}$ H. Oberlack, ${ }^{113}$ T. Obermann, ${ }^{24}$ J. Ocariz, ${ }^{132}$ A. Ochi, ${ }^{80}$ I. Ochoa, ${ }^{38}$ J. P. Ochoa-Ricoux, ${ }^{144 a}$ K. O'Connor, ${ }^{26}$ S. Oda ${ }^{85}$ S. Odaka, ${ }^{79}$ A. Oh,${ }^{98}$ S. H. Oh, ${ }^{47}$ C. C. Ohm, ${ }^{151}$ H. Oide, ${ }^{53 b, 53 a}$ H. Okawa, ${ }^{166}$ Y. Okazaki, ${ }^{83}$ Y. Okumura, ${ }^{160}$ T. Okuyama, ${ }^{79}$ A. Olariu, ${ }^{27 b}$ L. F. Oleiro Seabra, ${ }^{136 a}$ S. A. Olivares Pino, ${ }^{144 a}$ D. Oliveira Damazio, ${ }^{29}$ J. L. Oliver, ${ }^{1}$ M. J. R. Olsson, ${ }^{36}$ A. Olszewski, ${ }^{82}$ J. Olszowska, ${ }^{82}$ D. C. O’Neil, ${ }^{149}$ A. Onofre, ${ }^{136 a, 136 e}$ K. Onogi, ${ }^{115}$ P. U. E. Onyisi, ${ }^{11}$ H. Oppen, ${ }^{130}$ M. J. Oreglia, ${ }^{36}$ Y. Oren, ${ }^{158}$ D. Orestano,${ }^{72 a, 72 b}$ E. C. Orgill, ${ }^{98}$ N. Orlando, ${ }^{61 b}$ A. A. O'Rourke, ${ }^{44}$ R. S. Orr, ${ }^{164}$ B. Osculati, ${ }^{53 b, 53 a, a}$ V. O'Shea, ${ }^{55}$ R. Ospanov ${ }^{58 a}$ G. Otero y Garzon, ${ }^{30}$ H. Otono, ${ }^{85}$ M. Ouchrif, ${ }^{34 d}$ F. Ould-Saada, ${ }^{130}$ A. Ouraou, ${ }^{142}$ Q. Ouyang, ${ }^{15 a}$ M. Owen, ${ }^{55}$ R. E. Owen, ${ }^{21}$ V. E. Ozcan, ${ }^{12 c}$ N. Ozturk, ${ }^{8}$ J. Pacalt, ${ }^{126}$ H. A. Pacey, ${ }^{31}$ K. Pachal,${ }^{149}$ A. Pacheco Pages,${ }^{14}$ L. Pacheco Rodriguez ${ }^{142}$ C. Padilla Aranda, ${ }^{14}$ S. Pagan Griso, ${ }^{18}$ M. Paganini, ${ }^{180}$ G. Palacino, ${ }^{63}$ S. Palazzo, ${ }^{40 b, 40 a}$ S. Palestini, ${ }^{35}$ M. Palka, ${ }^{81 b}$ D. Pallin, ${ }^{37}$ I. Panagoulias, ${ }^{10}$ C. E. Pandini, ${ }^{35}$ J. G. Panduro Vazquez, ${ }^{91}$ P. Pani,${ }^{35}$ G. Panizzo, ${ }^{64 a, 64 c}$ L. Paolozzi, ${ }^{52}$ T. D. Papadopoulou, ${ }^{10}$

K. Papageorgiou, ${ }^{9, t}$ A. Paramonov, ${ }^{6}$ D. Paredes Hernandez, ${ }^{61 \mathrm{~b}}$ S. R. Paredes Saenz, ${ }^{131}$ B. Parida, ${ }^{58 \mathrm{c}}$ A. J. Parker, ${ }^{87}$ K. A. Parker, ${ }^{44}$ M. A. Parker, ${ }^{31}$ F. Parodi,${ }^{53 b, 53 a}$ J. A. Parsons, ${ }^{38}$ U. Parzefall, ${ }^{50}$ V. R. Pascuzzi, ${ }^{164}$ J. M. P. Pasner, ${ }^{143}$ E. Pasqualucci ${ }^{70 a}$ S. Passaggio,${ }^{53 b}$ F. Pastore, ${ }^{91}$ P. Pasuwan, ${ }^{43 a, 43 b}$ S. Pataraia, ${ }^{97}$ J. R. Pater, ${ }^{98}$ A. Pathak, ${ }^{178, f}$ T. Pauly, ${ }^{35}$ B. Pearson, ${ }^{113}$ M. Pedersen, ${ }^{130}$ L. Pedraza Diaz,${ }^{117}$ S. Pedraza Lopez,${ }^{171}$ R. Pedro, ${ }^{136 a, 136 b}$ S. V. Peleganchuk, ${ }^{120 b, 120 a}$ O. Penc, ${ }^{137}$ C. Peng, ${ }^{15 d}$ H. Peng, ${ }^{58 a}$ B. S. Peralva, ${ }^{78 a}$ M. M. Perego, ${ }^{142}$ A. P. Pereira Peixoto, ${ }^{136 a}$ D. V. Perepelitsa, ${ }^{29}$ F. Peri, ${ }^{19}$ L. Perini, ${ }^{66 a, 66 b}$ H. Pernegger, ${ }^{35}$ S. Perrella, ${ }^{67,67 b}$ V. D. Peshekhonov, ${ }^{77, a}$ K. Peters,${ }^{44}$ R. F. Y. Peters,${ }^{98}$ B. A. Petersen, ${ }^{35}$

T. C. Petersen, ${ }^{39}$ E. Petit, ${ }^{56}$ A. Petridis, ${ }^{1}$ C. Petridou, ${ }^{159}$ P. Petroff,,${ }^{128}$ E. Petrolo,${ }^{70 a}$ M. Petrov,${ }^{131}$ F. Petrucci, ${ }^{72 a, 72 b}$ M. Pettee, ${ }^{180}$ N. E. Pettersson, ${ }^{100}$ A. Peyaud, ${ }^{142}$ R. Pezoa, ${ }^{144 b}$ T. Pham,${ }^{102}$ F. H. Phillips,${ }^{104}$ P. W. Phillips,${ }^{141}$ G. Piacquadio, ${ }^{152}$ E. Pianori, ${ }^{18}$ A. Picazio, ${ }^{100}$ M. A. Pickering, ${ }^{131}$ R. Piegaia,${ }^{30}$ J. E. Pilcher ${ }^{36}$ A. D. Pilkington, ${ }^{98}$ M. Pinamonti, ${ }^{71 \mathrm{a}, 71 \mathrm{~b}}$ J. L. Pinfold, ${ }^{3}$ M. Pitt,,${ }^{177}$ M-A. Pleier ${ }^{29}$ V. Pleskot, ${ }^{139}$ E. Plotnikova, ${ }^{77}$ D. Pluth,${ }^{76}$ P. Podberezko, ${ }^{120 b, 120 a}$ R. Poettgen, ${ }^{94}$ R. Poggi,${ }^{52}$ L. Poggioli, ${ }^{128}$ I. Pogrebnyak, ${ }^{104}$ D. Pohl,${ }^{24}$ I. Pokharel,${ }^{51}$ G. Polesello, ${ }^{68 a}$ A. Poley, ${ }^{44}$ A. Policicchio, ${ }^{40 b, 40 a}$ R. Polifka, ${ }^{35}$ A. Polini,${ }^{23 b}$ C. S. Pollard,${ }^{44}$ V. Polychronakos,${ }^{29}$ D. Ponomarenko, ${ }^{110}$ L. Pontecorvo, ${ }^{70 a}$ G. A. Popeneciu, ${ }^{27 d}$ D. M. Portillo Quintero, ${ }^{132}$ S. Pospisil, ${ }^{138}$ K. Potamianos, ${ }^{44}$ I. N. Potrap,${ }^{77}$ C. J. Potter, ${ }^{31}$ H. Potti, ${ }^{11}$ T. Poulsen, ${ }^{94}$ J. Poveda ${ }^{35}$ T. D. Powell, ${ }^{146}$ M. E. Pozo Astigarraga,${ }^{35}$ P. Pralavorio, ${ }^{99}$ S. Prell,${ }^{76}$ D. Price, ${ }^{98}$ M. Primavera, ${ }^{65 a}$ S. Prince, ${ }^{101}$ N. Proklova, ${ }^{110}$ K. Prokofiev, ${ }^{61 \mathrm{c}}$ F. Prokoshin, ${ }^{144 \mathrm{~b}}$ S. Protopopescu, ${ }^{29}$ J. Proudfoot, ${ }^{6}$ M. Przybycien, ${ }^{81 a}$ A. Puri, ${ }^{170}$ P. Puzo, ${ }^{128}$ J. Qian, ${ }^{103}$ Y. Qin, ${ }^{98}$ A. Quadt ${ }^{51}$ M. Queitsch-Maitland, ${ }^{44}$ A. Qureshi, ${ }^{1}$ P. Rados,${ }^{102}$ F. Ragusa, ${ }^{66 a, 66 b}$ G. Rahal, ${ }^{95}$ J. A. Raine, ${ }^{98}$ S. Rajagopalan, ${ }^{29}$ A. Ramirez Morales, ${ }^{90}$ T. Rashid, ${ }^{128}$ S. Raspopov, ${ }^{5}$ M. G. Ratti, ${ }^{66 a, 66 b}$ D. M. Rauch, ${ }^{44}$ F. Rauscher, ${ }^{112}$ S. Rave, ${ }^{97}$ B. Ravina ${ }^{146}$ I. Ravinovich, ${ }^{177}$ J. H. Rawling, ${ }^{98}$ M. Raymond, ${ }^{35}$ A. L. Read, ${ }^{130}$ N. P. Readioff, ${ }^{56}$ M. Reale, ${ }^{65 a, 65 b}$ D. M. Rebuzzi, ${ }^{68 a, 68 b}$ A. Redelbach, ${ }^{174}$ G. Redlinger, ${ }^{29}$ R. Reece, ${ }^{143}$ R. G. Reed, ${ }^{32 c}$

K. Reeves, ${ }^{42}$ L. Rehnisch, ${ }^{19}$ J. Reichert, ${ }^{133}$ A. Reiss, ${ }^{97}$ C. Rembser,${ }^{35}$ H. Ren, ${ }^{15 \mathrm{~d}}$ M. Rescigno, ${ }^{70 a}$ S. Resconi, ${ }^{66 a}$ E. D. Resseguie, ${ }^{133}$ S. Rettie, ${ }^{172}$ E. Reynolds, ${ }^{21}$ O. L. Rezanova, ${ }^{120 b, 120 a}$ P. Reznicek, ${ }^{139}$ R. Richter, ${ }^{113}$ S. Richter, ${ }^{92}$ E. Richter-Was, ${ }^{81 b}$ O. Ricken, ${ }^{24}$ M. Ridel, ${ }^{132}$ P. Rieck, ${ }^{113}$ C. J. Riegel, ${ }^{179}$ O. Rifki, ${ }^{44}$ M. Rijssenbeek, ${ }^{152}$ A. Rimoldi, ${ }^{68 a, 68 b}$ M. Rimoldi, ${ }^{20}$ L. Rinaldi, ${ }^{23 b}$ G. Ripellino, ${ }^{151}$ B. Ristić, ${ }^{87}$ E. Ritsch,${ }^{35}$ I. Riu, ${ }^{14}$ J. C. Rivera Vergara, ${ }^{144 a}$ F. Rizatdinova, ${ }^{125}$ E. Rizvi, ${ }^{90}$ C. Rizzi, ${ }^{14}$ R. T. Roberts, ${ }^{98}$ S. H. Robertson, ${ }^{101, n}$ A. Robichaud-Veronneau, ${ }^{101}$ D. Robinson, ${ }^{31}$

J. E. M. Robinson, ${ }^{44}$ A. Robson,${ }^{55}$ E. Rocco, ${ }^{97}$ C. Roda,${ }^{69,69 b}$ Y. Rodina, ${ }^{99}$ S. Rodriguez Bosca, ${ }^{171}$ A. Rodriguez Perez,${ }^{14}$ D. Rodriguez Rodriguez, ${ }^{171}$ A. M. Rodríguez Vera ${ }^{165 b}$ S. Roe, ${ }^{35}$ C. S. Rogan, ${ }^{57}$ O. Røhne, ${ }^{130}$ R. Röhrig, ${ }^{113}$

C. P. A. Roland, ${ }^{63}$ J. Roloff, ${ }^{57}$ A. Romaniouk,${ }^{110}$ M. Romano,${ }^{23 b, 23 a}$ N. Rompotis, ${ }^{88}$ M. Ronzani, ${ }^{121}$ L. Roos, ${ }^{132}$ S. Rosati, ${ }^{70 a}$ K. Rosbach ${ }^{50}$ P. Rose, ${ }^{143}$ N-A. Rosien, ${ }^{51}$ E. Rossi ${ }^{67 a, 67 b}$ L. P. Rossi ${ }^{53 b}$ L. Rossini, ${ }^{66 a, 66 b}$ J. H. N. Rosten, ${ }^{31}$ R. Rosten, ${ }^{14}$ M. Rotaru, ${ }^{27 b}$ J. Rothberg, ${ }^{145}$ D. Rousseau, ${ }^{128}$ D. Roy ${ }^{32 \mathrm{c}}$ A. Rozanov, ${ }^{99}$ Y. Rozen, ${ }^{157}$ X. Ruan, ${ }^{32 \mathrm{c}}$ F. Rubbo, ${ }^{150}$ F. Rühr, ${ }^{50}$ A. Ruiz-Martinez, ${ }^{33}$ Z. Rurikova, ${ }^{50}$ N. A. Rusakovich, ${ }^{77}$ H. L. Russell, ${ }^{101}$ J. P. Rutherfoord, ${ }^{7}$ N. Ruthmann, ${ }^{35}$

E. M. Rüttinger ${ }^{44, m m}$ Y. F. Ryabov, ${ }^{134}$ M. Rybar, ${ }^{170}$ G. Rybkin, ${ }^{128}$ S. Ryu, ${ }^{6}$ A. Ryzhov, ${ }^{140}$ G. F. Rzehorz, ${ }^{51}$ P. Sabatini,${ }^{51}$ G. Sabato, ${ }^{118}$ S. Sacerdoti, ${ }^{128}$ H. F-W. Sadrozinski, ${ }^{143}$ R. Sadykov, ${ }^{77}$ F. Safai Tehrani, ${ }^{70 a}$ P. Saha, ${ }^{119}$ M. Sahinsoy, ${ }^{59 a}$ 
A. Sahu, ${ }^{179}$ M. Saimpert, ${ }^{44}$ M. Saito, ${ }^{160}$ T. Saito, ${ }^{160}$ H. Sakamoto, ${ }^{160}$ A. Sakharov, ${ }^{121, f f}$ D. Salamani, ${ }^{52}$ G. Salamanna, ${ }^{72 a, 72 b}$ J. E. Salazar Loyola, ${ }^{144 b}$ D. Salek, ${ }^{118}$ P. H. Sales De Bruin, ${ }^{169}$ D. Salihagic, ${ }^{113}$ A. Salnikov, ${ }^{150}$ J. Salt, ${ }^{171}$ D. Salvatore, ${ }^{40 b, 40 a}$ F. Salvatore, ${ }^{153}$ A. Salvucci, ${ }^{61 \mathrm{a}, 61 \mathrm{~b}, 61 \mathrm{c}}$ A. Salzburger, ${ }^{35}$ D. Sammel, ${ }^{50}$ D. Sampsonidis, ${ }^{159}$ D. Sampsonidou, ${ }^{159}$ J. Sánchez, ${ }^{171}$ A. Sanchez Pineda, ${ }^{64 a, 64 c}$ H. Sandaker, ${ }^{130}$ C. O. Sander, ${ }^{44}$ M. Sandhoff, ${ }^{179}$ C. Sandoval, ${ }^{22}$ D. P. C. Sankey, ${ }^{141}$ M. Sannino, ${ }^{53 b, 53 a}$ Y. Sano, ${ }^{115}$ A. Sansoni, ${ }^{49}$ C. Santoni, ${ }^{37}$ H. Santos ${ }^{136 a}$ I. Santoyo Castillo, ${ }^{153}$ A. Sapronov, ${ }^{77}$ J. G. Saraiva, ${ }^{136 a, 136 d}$ O. Sasaki, ${ }^{79}$ K. Sato, ${ }^{166}$ E. Sauvan, ${ }^{5}$ P. Savard, ${ }^{164, e}$ N. Savic, ${ }^{113}$ R. Sawada, ${ }^{160}$ C. Sawyer, ${ }^{141}$ L. Sawyer, ${ }^{93, v}$ C. Sbarra, ${ }^{23 b}$ A. Sbrizzi, ${ }^{23 b, 23 a}$ T. Scanlon, ${ }^{92}$ J. Schaarschmidt, ${ }^{145}$ P. Schacht, ${ }^{113}$ B. M. Schachtner, ${ }^{112}$ D. Schaefer, ${ }^{36}$ L. Schaefer, ${ }^{133}$ J. Schaeffer, ${ }^{97}$ S. Schaepe, ${ }^{35}$ U. Schäfer,${ }^{97}$ A. C. Schaffer,${ }^{128}$ D. Schaile, ${ }^{112}$ R. D. Schamberger, ${ }^{152}$ N. Scharmberg, ${ }^{98}$ V. A. Schegelsky, ${ }^{134}$ D. Scheirich, ${ }^{139}$ F. Schenck, ${ }^{19}$ M. Schernau, ${ }^{168}$ C. Schiavi, ${ }^{53 b, 53 a}$ S. Schier, ${ }^{143}$ L. K. Schildgen, ${ }^{24}$ Z. M. Schillaci ${ }^{26}$ E. J. Schioppa, ${ }^{35}$ M. Schioppa, ${ }^{40 b, 40 a}$ K. E. Schleicher, ${ }^{50}$ S. Schlenker, ${ }^{35}$ K. R. Schmidt-Sommerfeld, ${ }^{113}$ K. Schmieden, ${ }^{35}$ C. Schmitt, ${ }^{97}$ S. Schmitt, ${ }^{44}$ S. Schmitz, ${ }^{97}$ U. Schnoor, ${ }^{50}$ L. Schoeffel, ${ }^{142}$ A. Schoening, ${ }^{59 b}$ E. Schopf,${ }^{24}$ M. Schott, ${ }^{97}$ J. F. P. Schouwenberg, ${ }^{117}$ J. Schovancova, ${ }^{35}$ S. Schramm, ${ }^{52}$ A. Schulte, ${ }^{97}$ H-C. Schultz-Coulon, ${ }^{59 a}$ M. Schumacher, ${ }^{50}$ B. A. Schumm, ${ }^{143}$ Ph. Schune, ${ }^{142}$ A. Schwartzman, ${ }^{150}$

T. A. Schwarz, ${ }^{103}$ H. Schweiger, ${ }^{98}$ Ph. Schwemling, ${ }^{142}$ R. Schwienhorst, ${ }^{104}$ A. Sciandra, ${ }^{24}$ G. Sciolla, ${ }^{26}$ M. Scornajenghi ${ }^{40 b, 40 a}$ F. Scuri ${ }^{69 a}$ F. Scutti, ${ }^{102}$ L. M. Scyboz,${ }^{113}$ J. Searcy, ${ }^{103}$ C. D. Sebastiani, ${ }^{70 a, 70 b}$ P. Seema, ${ }^{24}$ S. C. Seidel, ${ }^{116}$ A. Seiden, ${ }^{143}$ T. Seiss, ${ }^{36}$ J. M. Seixas, ${ }^{78 b}$ G. Sekhniaidze, ${ }^{67 a}$ K. Sekhon, ${ }^{103}$ S. J. Sekula, ${ }^{41}$ N. Semprini-Cesari, ${ }^{23 b, 23 a}$ S. Sen, ${ }^{47}$ S. Senkin, ${ }^{37}$ C. Serfon, ${ }^{130}$ L. Serin, ${ }^{128}$ L. Serkin, ${ }^{64 a, 64 b}$ M. Sessa, ${ }^{72 a, 72 b}$ H. Severini, ${ }^{124}$ F. Sforza, ${ }^{167}$ A. Sfyrla, ${ }^{52}$ E. Shabalina, ${ }^{51}$ J. D. Shahinian, ${ }^{143}$ N. W. Shaikh, ${ }^{43 a, 43 b}$ L. Y. Shan, ${ }^{15 a}$ R. Shang, ${ }^{170}$ J. T. Shank, ${ }^{25}$ M. Shapiro, ${ }^{18}$ A. S. Sharma, ${ }^{1}$ A. Sharma, ${ }^{131}$ P. B. Shatalov, ${ }^{109}$ K. Shaw, ${ }^{153}$ S. M. Shaw, ${ }^{98}$ A. Shcherbakova, ${ }^{134}$ Y. Shen, ${ }^{124}$ N. Sherafati, ${ }^{33}$ A. D. Sherman, ${ }^{25}$ P. Sherwood, ${ }^{92}$ L. Shi, ${ }^{155, n n}$ S. Shimizu, ${ }^{80}$ C. O. Shimmin, ${ }^{180}$ M. Shimojima, ${ }^{114}$ I. P. J. Shipsey, ${ }^{131}$ S. Shirabe,${ }^{85}$ M. Shiyakova, ${ }^{77}$ J. Shlomi,${ }^{177}$ A. Shmeleva ${ }^{108}$ D. Shoaleh Saadi, ${ }^{107}$ M. J. Shochet, ${ }^{36}$ S. Shojaii, ${ }^{102}$ D. R. Shope, ${ }^{124}$ S. Shrestha, ${ }^{122}$ E. Shulga, ${ }^{110}$ P. Sicho, ${ }^{137}$ A. M. Sickles, ${ }^{170}$ P. E. Sidebo, ${ }^{151}$

E. Sideras Haddad, ${ }^{32 \mathrm{c}}$ O. Sidiropoulou, ${ }^{174}$ A. Sidoti, ${ }^{23 b, 23 a}$ F. Siegert, ${ }^{46}$ Dj. Sijacki, ${ }^{16}$ J. Silva, ${ }^{136 a}$ M. Silva Jr., ${ }^{178}$ M. V. Silva Oliveira, ${ }^{78 a}$ S. B. Silverstein, ${ }^{43 a}$ L. Simic, ${ }^{77}$ S. Simion, ${ }^{128}$ E. Simioni, ${ }^{97}$ M. Simon, ${ }^{97}$ P. Sinervo, ${ }^{164}$ N. B. Sinev, ${ }^{127}$ M. Sioli, ${ }^{23 b, 23 a}$ G. Siragusa, ${ }^{174}$ I. Siral, ${ }^{103}$ S. Yu. Sivoklokov, ${ }^{111}$ J. Sjölin, ${ }^{43 a, 43 b}$ M. B. Skinner, ${ }^{87}$ P. Skubic, ${ }^{124}$ M. Slater, ${ }^{21}$ T. Slavicek, ${ }^{138}$ M. Slawinska, ${ }^{82}$ K. Sliwa, ${ }^{167}$ R. Slovak, ${ }^{139}$ V. Smakhtin, ${ }^{177}$ B. H. Smart, ${ }^{5}$ J. Smiesko, ${ }^{28 a}$ N. Smirnov, ${ }^{110}$ S. Yu. Smirnov, ${ }^{110}$ Y. Smirnov, ${ }^{110}$ L. N. Smirnova, ${ }^{111}$ O. Smirnova, ${ }^{94}$ J. W. Smith, ${ }^{51}$ M. N. K. Smith, ${ }^{38}$ R. W. Smith, ${ }^{38}$ M. Smizanska, ${ }^{87}$ K. Smolek, ${ }^{138}$ A. A. Snesarev, ${ }^{108}$ I. M. Snyder, ${ }^{127}$ S. Snyder, ${ }^{29}$ R. Sobie, ${ }^{173, n}$ A. M. Soffa, ${ }^{168}$ A. Soffer, ${ }^{158}$ A. Søgaard, ${ }^{48}$ D. A. Soh, ${ }^{155}$ G. Sokhrannyi, ${ }^{89}$ C. A. Solans Sanchez, ${ }^{35}$ M. Solar,${ }^{138}$ E. Yu. Soldatov, ${ }^{110}$ U. Soldevila, ${ }^{171}$ A. A. Solodkov, ${ }^{140}$ A. Soloshenko, ${ }^{77}$ O. V. Solovyanov, ${ }^{140}$ V. Solovyev, ${ }^{134}$ P. Sommer, ${ }^{146}$ H. Son, ${ }^{167}$ W. Song, ${ }^{141}$

A. Sopczak, ${ }^{138}$ F. Sopkova, ${ }^{28 b}$ D. Sosa, ${ }^{59 b}$ C. L. Sotiropoulou, ${ }^{69 a, 69 b}$ S. Sottocornola, ${ }^{68 a, 68 b}$ R. Soualah, ${ }^{64 a, 64 c, 0 o}$ A. M. Soukharev, ${ }^{120 b, 120 \mathrm{a}}$ D. South, ${ }^{44}$ B. C. Sowden, ${ }^{91}$ S. Spagnolo, ${ }^{65 a, 65 b}$ M. Spalla, ${ }^{113}$ M. Spangenberg, ${ }^{175}$ F. Spanò ${ }^{91}$ D. Sperlich, ${ }^{19}$ F. Spettel, ${ }^{113}$ T. M. Spieker, ${ }^{59 a}$ R. Spighi, ${ }^{23 b}$ G. Spigo,${ }^{35}$ L. A. Spiller, ${ }^{102}$ D. P. Spiteri, ${ }^{55}$ M. Spousta, ${ }^{139}$ A. Stabile, ${ }^{66 a, 66 b}$ R. Stamen ${ }^{59 a}$ S. Stamm, ${ }^{19}$ E. Stanecka,${ }^{82}$ R. W. Stanek, ${ }^{6}$ C. Stanescu, ${ }^{72 a}$ B. Stanislaus, ${ }_{131}$ M. M. Stanitzki, ${ }^{44}$ B. Stapf, ${ }^{118}$ S. Stapnes, ${ }^{130}$ E. A. Starchenko, ${ }^{140}$ G. H. Stark, ${ }^{36}$ J. Stark, ${ }^{56}$ S. H Stark, ${ }^{39}$ P. Staroba, ${ }^{137}$ P. Starovoitov, ${ }^{59 a}$ S. Stärz, ${ }^{35}$ R. Staszewski, ${ }^{82}$ M. Stegler, ${ }^{44}$ P. Steinberg, ${ }^{29}$ B. Stelzer, ${ }^{149}$ H. J. Stelzer, ${ }^{35}$ O. Stelzer-Chilton, ${ }^{165 a}$ H. Stenzel, ${ }^{54}$ T. J. Stevenson, ${ }^{90}$ G. A. Stewart, ${ }^{55}$ M. C. Stockton, ${ }^{127}$ G. Stoicea, ${ }^{27 b}$ P. Stolte, ${ }^{51}$ S. Stonjek, ${ }^{113}$ A. Straessner, ${ }^{46}$

J. Strandberg, ${ }^{151}$ S. Strandberg, ${ }^{43 a, 43 b}$ M. Strauss,${ }^{124}$ P. Strizenec, ${ }^{28 b}$ R. Ströhmer, ${ }^{174}$ D. M. Strom, ${ }^{127}$ R. Stroynowski, ${ }^{41}$ A. Strubig, ${ }^{48}$ S. A. Stucci, ${ }^{29}$ B. Stugu, ${ }^{17}$ J. Stupak, ${ }^{124}$ N. A. Styles, ${ }^{44}$ D. Su, ${ }^{150}$ J. Su, ${ }^{135}$ S. Suchek, ${ }^{59 a}$ Y. Sugaya, ${ }^{129}$ M. Suk, ${ }^{138}$ V. V. Sulin, ${ }^{108}$ D. M. S. Sultan, ${ }^{52}$ S. Sultansoy, ${ }^{4 \mathrm{c}}$ T. Sumida ${ }^{83}$ S. Sun, ${ }^{103}$ X. Sun, ${ }^{3}$ K. Suruliz, ${ }^{153}$ C. J. E. Suster, ${ }^{154}$ M. R. Sutton, ${ }^{153}$ S. Suzuki ${ }^{79}$ M. Svatos, ${ }^{137}$ M. Swiatlowski, ${ }^{36}$ S. P. Swift, ${ }^{2}$ A. Sydorenko, ${ }^{97}$ I. Sykora ${ }^{28 a}$ T. Sykora, ${ }^{139}$ D. Ta, ${ }^{97}$ K. Tackmann, ${ }^{44, p p}$ J. Taenzer, ${ }^{158}$ A. Taffard, ${ }^{168}$ R. Tafirout, ${ }^{165}$ E. Tahirovic,${ }^{90}$ N. Taiblum, ${ }^{158}$ H. Takai, ${ }^{29}$ R. Takashima ${ }^{84}$ E. H. Takasugi, ${ }^{113}$ K. Takeda, ${ }^{80}$ T. Takeshita, ${ }^{147}$ Y. Takubo, ${ }^{79}$ M. Talby, ${ }^{99}$ A. A. Talyshev, ${ }^{120 b, 120 a}$ J. Tanaka, ${ }^{160}$ M. Tanaka, ${ }^{162}$ R. Tanaka, ${ }^{128}$ R. Tanioka, ${ }^{80}$ B. B. Tannenwald, ${ }^{122}$ S. Tapia Araya ${ }^{144 b}$ S. Tapprogge, ${ }^{97}$ A. Tarek Abouelfadl Mohamed, ${ }^{132}$ S. Tarem, ${ }^{157}$ G. Tarna, ${ }^{27 b, q}$ G. F. Tartarelli, ${ }^{66 a}$ P. Tas, ${ }^{139}$ M. Tasevsky, ${ }^{137}$ T. Tashiro, ${ }^{83}$ E. Tassi, ${ }^{40 b, 40 a}$ A. Tavares Delgado, ${ }^{136 a, 136 b}$ Y. Tayalati, ${ }^{34 \mathrm{e}}$ A. C. Taylor, ${ }^{116}$ A. J. Taylor, ${ }^{48}$ G. N. Taylor, ${ }^{102}$ P. T. E. Taylor, ${ }^{102}$ W. Taylor, ${ }^{165 b}$ A. S. Tee, ${ }^{87}$ P. Teixeira-Dias, ${ }^{91}$ D. Temple, ${ }^{149}$ H. Ten Kate,${ }^{35}$ P. K. Teng, ${ }^{155}$ J. J. Teoh, ${ }^{129}$ F. Tepel, ${ }^{179}$ S. Terada ${ }^{79}$ K. Terashi, ${ }^{160}$ J. Terron, ${ }^{96}$ S. Terzo, ${ }^{14}$ M. Testa, ${ }^{49}$ R. J. Teuscher, ${ }^{164, n}$ S. J. Thais, ${ }^{180}$ T. Theveneaux-Pelzer, ${ }^{44}$ F. Thiele, ${ }^{39}$ J. P. Thomas, ${ }^{21}$ A. S. Thompson, ${ }^{55}$ P. D. Thompson, ${ }^{21}$ L. A. Thomsen, ${ }^{180}$ E. Thomson, ${ }^{133}$ Y. Tian, ${ }^{38}$ 
R. E. Ticse Torres, ${ }^{51}$ V. O. Tikhomirov, ${ }^{108, q q}$ Yu. A. Tikhonov, ${ }^{120 b, 120 a}$ S. Timoshenko, ${ }^{110}$ P. Tipton, ${ }^{180}$ S. Tisserant, ${ }^{99}$ K. Todome, ${ }^{162}$ S. Todorova-Nova, ${ }^{5}$ S. Todt, ${ }^{46}$ J. Tojo, ${ }^{85}$ S. Tokár, ${ }^{28 a}$ K. Tokushuku, ${ }^{79}$ E. Tolley, ${ }^{122}$ K. G. Tomiwa, ${ }^{32 c}$ M. Tomoto, ${ }^{115}$ L. Tompkins, ${ }^{150}$ K. Toms, ${ }^{116}$ B. Tong, ${ }^{57}$ P. Tornambe, ${ }^{50}$ E. Torrence, ${ }^{127}$ H. Torres, ${ }^{46}$ E. Torró Pastor, ${ }^{145}$ C. Tosciri, ${ }^{131}$ J. Toth, ${ }^{99, r r}$ F. Touchard, ${ }^{99}$ D. R. Tovey, ${ }^{146}$ C. J. Treado, ${ }^{121}$ T. Trefzger, ${ }^{174}$ F. Tresoldi, ${ }^{153}$ A. Tricoli, ${ }^{29}$ I. M. Trigger, ${ }^{165 a}$ S. Trincaz-Duvoid, ${ }^{132}$ M. F. Tripiana, ${ }^{14}$ W. Trischuk, ${ }^{164}$ B. Trocmé, ${ }^{56}$ A. Trofymov, ${ }^{128}$ C. Troncon, ${ }^{66 a}$ M. Trovatelli, ${ }^{173}$ F. Trovato, ${ }^{153}$ L. Truong, ${ }^{32 b}$ M. Trzebinski, ${ }^{82}$ A. Trzupek, ${ }^{82}$ F. Tsai, ${ }^{44}$ J. C-L. Tseng, ${ }^{131}$ P. V. Tsiareshka, ${ }^{105}$ N. Tsirintanis, ${ }^{9}$ V. Tsiskaridze, ${ }^{152}$ E. G. Tskhadadze, ${ }^{156 a}$ I. I. Tsukerman, ${ }^{109}$ V. Tsulaia, ${ }^{18}$ S. Tsuno, ${ }^{79}$ D. Tsybychev, ${ }^{152}$ Y. Tu, ${ }^{61 b}$ A. Tudorache, ${ }^{27 b}$ V. Tudorache, ${ }^{27 b}$ T. T. Tulbure, ${ }^{27 a}$ A. N. Tuna, ${ }^{57}$ S. Turchikhin, ${ }^{77}$ D. Turgeman, ${ }^{177}$ I. Turk Cakir, ${ }^{4 b, s s}$ R. Turra, ${ }^{66 a}$ P. M. Tuts, ${ }^{38}$ E. Tzovara, ${ }^{97}$ G. Ucchielli, ${ }^{23 b, 23 a}$ I. Ueda, ${ }^{79}$ M. Ughetto, ${ }^{43 a, 43 b}$ F. Ukegawa, ${ }^{166}$ G. Unal, ${ }^{35}$ A. Undrus, ${ }^{29}$ G. Unel, ${ }^{168}$ F. C. Ungaro, ${ }^{102}$ Y. Unno, ${ }^{79}$ K. Uno, ${ }^{160}$ J. Urban, $^{28 b}$ P. Urquijo, ${ }^{102}$ P. Urrejola, ${ }^{97}$ G. Usai, ${ }^{8}$ J. Usui, ${ }^{79}$ L. Vacavant, ${ }^{99}$ V. Vacek, ${ }^{138}$ B. Vachon, ${ }^{101}$ K. O. H. Vadla, ${ }^{130}$ A. Vaidya, ${ }^{92}$ C. Valderanis, ${ }^{112}$

E. Valdes Santurio, ${ }^{43 a, 43 b}$ M. Valente, ${ }^{52}$ S. Valentinetti, ${ }^{23 b, 23 a}$ A. Valero, ${ }^{171}$ L. Valéry, ${ }^{44}$ R. A. Vallance, ${ }^{21}$ A. Vallier, ${ }^{5}$ J. A. Valls Ferrer, ${ }^{171}$ T. R. Van Daalen, ${ }^{14}$ W. Van Den Wollenberg, ${ }^{118}$ H. Van der Graaf, ${ }^{118}$ P. Van Gemmeren, ${ }^{6}$ J. Van Nieuwkoop, ${ }^{149}$ I. Van Vulpen, ${ }^{118}$ M. C. van Woerden, ${ }^{118}$ M. Vanadia, ${ }^{71 \mathrm{a}, 71 \mathrm{~b}}$ W. Vandelli, ${ }^{35}$ A. Vaniachine, ${ }^{163}$ P. Vankov, ${ }^{118}$ R. Vari, ${ }^{70 a}$ E. W. Varnes, ${ }^{7}$ C. Varni, ${ }^{53 b, 53 a}$ T. Varol, ${ }^{41}$ D. Varouchas, ${ }^{128}$ A. Vartapetian, ${ }^{8}$ K. E. Varvell, ${ }^{154}$ G. A. Vasquez, ${ }^{144 b}$ J. G. Vasquez, ${ }^{180}$ F. Vazeille, ${ }^{37}$ D. Vazquez Furelos, ${ }^{14}$ T. Vazquez Schroeder, ${ }^{101}$ J. Veatch, ${ }^{51}$ V. Vecchio, ${ }^{72 a, 72 b}$ L. M. Veloce, ${ }^{164}$ F. Veloso, ${ }^{136 a, 136 c}$ S. Veneziano, ${ }^{70 a}$ A. Ventura, ${ }^{65 a, 65 b}$ M. Venturi, ${ }^{173}$ N. Venturi, ${ }^{35}$ V. Vercesi, ${ }^{68 \mathrm{a}}$ M. Verducci, ${ }^{72 a, 72 b}$ C. M. Vergel Infante, ${ }^{76}$ W. Verkerke, ${ }^{118}$ A. T. Vermeulen, ${ }^{118}$ J. C. Vermeulen, ${ }^{118}$ M. C. Vetterli, ${ }^{149, e}$ N. Viaux Maira, ${ }^{144 b}$ O. Viazlo, ${ }^{94}$ I. Vichou, ${ }^{170, a}$ T. Vickey, ${ }^{146}$ O. E. Vickey Boeriu, ${ }^{146}$ G. H. A. Viehhauser, ${ }^{131}$ S. Viel, ${ }^{18}$ L. Vigani, ${ }^{131}$ M. Villa, ${ }^{23 b, 23 a}$ M. Villaplana Perez, ${ }^{66 a, 66 b}$ E. Vilucchi, ${ }^{49}$ M. G. Vincter, ${ }^{33}$ V. B. Vinogradov, ${ }^{77}$ A. Vishwakarma, ${ }^{44}$ C. Vittori, ${ }^{23 b, 23 a}$ I. Vivarelli, ${ }^{153}$ S. Vlachos, ${ }^{10}$ M. Vogel, ${ }^{179}$ P. Vokac, ${ }^{138}$ G. Volpi, ${ }^{14}$ S. E. Von Buddenbrock, ${ }^{32 c}$ E. Von Toerne, ${ }^{24}$ V. Vorobel, ${ }^{139}$ K. Vorobev, ${ }^{110}$ M. Vos, ${ }^{171}$ J. H. Vossebeld, ${ }^{88}$ N. Vranjes, ${ }^{16}$

M. Vranjes Milosavljevic, ${ }^{16}$ V. Vrba, ${ }^{138}$ M. Vreeswijk, ${ }^{118}$ T. Šfiligoj, ${ }^{89}$ R. Vuillermet, ${ }^{35}$ I. Vukotic, $^{36}$ T. Ženišs $^{28 a}$

L. Živković, ${ }^{16}$ P. Wagner, ${ }^{24}$ W. Wagner, ${ }^{179}$ J. Wagner-Kuhr, ${ }^{112}$ H. Wahlberg, ${ }^{86}$ S. Wahrmund, ${ }^{46} \mathrm{~K}$. Wakamiya, ${ }^{80}$ V. M. Walbrecht, ${ }^{113}$ J. Walder, ${ }^{87}$ R. Walker, ${ }^{112}$ W. Walkowiak, ${ }^{148}$ V. Wallangen, ${ }^{43 a, 43 b}$ A. M. Wang, ${ }^{57}$ C. Wang, ${ }^{58 b, q}$ F. Wang, ${ }^{178}$ H. Wang, ${ }^{18}$ H. Wang, ${ }^{3}$ J. Wang, ${ }^{154}$ J. Wang, ${ }^{59 b}$ P. Wang, ${ }^{41}$ Q. Wang, ${ }^{124}$ R.-J. Wang, ${ }^{132}$ R. Wang, ${ }^{58 a}$ R. Wang, ${ }^{6}$ S. M. Wang, ${ }^{155}$ W. T. Wang, ${ }^{58 \mathrm{a}}$ W. Wang, ${ }^{155, \mathrm{tt}}$ W. X. Wang, ${ }^{58 \mathrm{a}, \mathrm{uu}}$ Y. Wang, ${ }^{58 \mathrm{a}}$ Z. Wang, ${ }^{58 \mathrm{c}}$ C. Wanotayaroj, ${ }^{44}$ A. Warburton, ${ }^{101}$ C. P. Ward, ${ }^{31}$ D. R. Wardrope, ${ }^{92}$ A. Washbrook, ${ }^{48}$ P. M. Watkins, ${ }^{21}$ A. T. Watson, ${ }^{21}$ M. F. Watson, ${ }^{21}$ G. Watts, ${ }^{145}$ S. Watts, ${ }^{98}$ B. M. Waugh, ${ }^{92}$ A. F. Webb, ${ }^{11}$ S. Webb, ${ }^{97}$ C. Weber, ${ }^{180}$ M. S. Weber, ${ }^{20}$ S. A. Weber, ${ }^{33}$ S. M. Weber, ${ }^{59 a}$ J. S. Webster, $^{6}$ A. R. Weidberg, ${ }^{131}$ B. Weinert, ${ }^{63}$ J. Weingarten, ${ }^{51}$ M. Weirich, ${ }^{97}$ C. Weiser, ${ }^{50}$ P. S. Wells, ${ }^{35}$ T. Wenaus, ${ }^{29}$ T. Wengler, ${ }^{35}$ S. Wenig, ${ }^{35}$ N. Wermes, ${ }^{24}$ M. D. Werner, ${ }^{76}$ P. Werner, ${ }^{35}$ M. Wessels, ${ }^{59}$ T. D. Weston, ${ }^{20}$ K. Whalen, ${ }^{127}$ N. L. Whallon, ${ }^{145}$ A. M. Wharton, ${ }^{87}$ A. S. White, ${ }^{103}$ A. White, ${ }^{8}$ M. J. White, ${ }^{1}$ R. White, ${ }^{144 b}$ D. Whiteson, ${ }^{168}$ B. W. Whitmore, ${ }^{87}$ F. J. Wickens, ${ }^{141}$ W. Wiedenmann, ${ }^{178}$ M. Wielers, ${ }^{141}$ C. Wiglesworth, ${ }^{39}$ L. A. M. Wiik-Fuchs, ${ }^{50}$ A. Wildauer, ${ }^{113}$ F. Wilk, ${ }^{98}$ H. G. Wilkens, ${ }^{35}$ L. J. Wilkins, ${ }^{91}$ H. H. Williams, ${ }^{133}$ S. Williams, ${ }^{31}$ C. Willis, ${ }^{104}$ S. Willocq, ${ }^{100}$ J. A. Wilson, ${ }^{21}$

I. Wingerter-Seez, ${ }^{5}$ E. Winkels, ${ }^{153}$ F. Winklmeier, ${ }^{127}$ O. J. Winston, ${ }^{153}$ B. T. Winter, ${ }^{24}$ M. Wittgen, ${ }^{150}$ M. Wobisch, ${ }^{93}$

A. Wolf, ${ }^{97}$ T. M. H. Wolf, ${ }^{118}$ R. Wolff, ${ }^{99}$ M. W. Wolter, ${ }^{82}$ H. Wolters, ${ }^{136 a, 136 c}$ V. W. S. Wong, ${ }^{172}$ N. L. Woods, ${ }^{143}$

S. D. Worm, ${ }^{21}$ B. K. Wosiek, ${ }^{82}$ K. W. Woźniak, ${ }^{82}$ K. Wraight, ${ }^{55}$ M. Wu, ${ }^{36}$ S. L. Wu, ${ }^{178}$ X. Wu, ${ }^{52}$ Y. Wu, ${ }^{58}$ T. R. Wyatt, ${ }^{98}$ B. M. Wynne, ${ }^{48}$ S. Xella, ${ }^{39}$ Z. Xi, ${ }^{103}$ L. Xia, ${ }^{175}$ D. Xu, ${ }^{15 a}$ H. Xu, ${ }^{58 a}$ L. Xu, ${ }^{29}$ T. Xu, ${ }^{142}$ W. Xu, ${ }^{103}$ B. Yabsley, ${ }^{154}$ S. Yacoob, ${ }^{32 a}$ K. Yajima, ${ }^{129}$ D. P. Yallup, ${ }^{92}$ D. Yamaguchi, ${ }^{162}$ Y. Yamaguchi, ${ }^{162}$ A. Yamamoto, ${ }^{79}$ T. Yamanaka, ${ }^{160}$ F. Yamane, ${ }^{80}$ M. Yamatani, ${ }^{160}$ T. Yamazaki, ${ }^{160}$ Y. Yamazaki, ${ }^{80}$ Z. Yan, ${ }^{25}$ H. J. Yang, ${ }^{58,58 d}$ H. T. Yang, ${ }^{18}$ S. Yang, ${ }^{75}$ Y. Yang, ${ }^{160}$ Z. Yang, ${ }^{17}$ W-M. Yao, ${ }^{18}$ Y. C. Yap, ${ }^{44}$ Y. Yasu, ${ }^{79}$ E. Yatsenko, ${ }^{58 c, 58 d}$ J. Ye, ${ }^{41}$ S. Ye, ${ }^{29}$ I. Yeletskikh, ${ }^{77}$ E. Yigitbasi, ${ }^{25}$ E. Yildirim, ${ }^{97}$ K. Yorita, ${ }^{176}$ K. Yoshihara, ${ }^{133}$ C. J. S. Young, ${ }^{35}$ C. Young, ${ }^{150}$ J. Yu, ${ }^{8}$ J. Yu, ${ }^{76}$ X. Yue, ${ }^{59 a}$ S. P. Y. Yuen, ${ }^{24}$ I. Yusuff, $^{31, v v}$ B. Zabinski, ${ }^{82}$ G. Zacharis, ${ }^{10}$ E. Zaffaroni, ${ }^{52}$ R. Zaidan, ${ }^{14}$ A. M. Zaitsev, ${ }^{140, k k}$ N. Zakharchuk, ${ }^{44}$ J. Zalieckas, ${ }^{17}$ S. Zambito, ${ }^{57}$ D. Zanzi, ${ }^{35}$ D. R. Zaripovas, ${ }^{55}$ S. V. Zeißner, ${ }^{45}$ C. Zeitnitz, ${ }^{179}$ G. Zemaityte, ${ }^{131}$ J. C. Zeng, ${ }^{170}$ Q. Zeng, ${ }^{150}$ O. Zenin, ${ }^{140}$ D. Zerwas, ${ }^{128}$ M. Zgubič, ${ }^{131}$ D. F. Zhang, ${ }^{58 b}$ D. Zhang, ${ }^{103}$ F. Zhang, ${ }^{178}$ G. Zhang, ${ }^{58 a, u u}$ H. Zhang, ${ }^{15 c}$ J. Zhang, ${ }^{6}$ L. Zhang, ${ }^{50}$ L. Zhang, ${ }^{58 \mathrm{a}}$ M. Zhang, ${ }^{170}$ P. Zhang, ${ }^{15 \mathrm{c}}$ R. Zhang, ${ }^{58 \mathrm{a}, \mathrm{q}}$ R. Zhang, ${ }^{24} \mathrm{X}$. Zhang, ${ }^{58 \mathrm{~b}}$ Y. Zhang, ${ }^{15 \mathrm{~d}}$ Z. Zhang, ${ }^{128} \mathrm{X}$. Zhao, ${ }^{41}$ Y. Zhao, ${ }^{58 b, 128, y}$ Z. Zhao, ${ }^{58 \mathrm{a}}$ A. Zhemchugov, ${ }^{77}$ B. Zhou, ${ }^{103}$ C. Zhou, ${ }^{178}$ L. Zhou, ${ }^{41}$ M. S. Zhou, ${ }^{15 d}$ M. Zhou, ${ }^{152}$ N. Zhou, ${ }^{58 c}$ Y. Zhou, ${ }^{7}$ C. G. Zhu, ${ }^{58 b}$ H. L. Zhu, ${ }^{58 a}$ H. Zhu, ${ }^{15 a}$ J. Zhu, ${ }^{103}$ Y. Zhu, ${ }^{58 a}$ X. Zhuang, ${ }^{15 a}$ K. Zhukov, ${ }^{108}$ V. Zhulanov, ${ }^{120 b, 120 a}$ 
A. Zibell, ${ }^{174}$ D. Zieminska, ${ }^{63}$ N. I. Zimine, ${ }^{77}$ S. Zimmermann,${ }^{50}$ Z. Zinonos, ${ }^{113}$ M. Zinser ${ }^{97}$ M. Ziolkowski, ${ }^{148}$ G. Zobernig, ${ }^{178}$ A. Zoccoli, ${ }^{23 b, 23 a}$ K. Zoch, ${ }^{51}$ T. G. Zorbas, ${ }^{146}$ R. Zou, ${ }^{36}$ M. Zur Nedden, ${ }^{19}$ and L. Zwalinski ${ }^{35}$

(ATLAS Collaboration)

${ }^{1}$ Department of Physics, University of Adelaide, Adelaide, Australia

${ }^{2}$ Physics Department, SUNY Albany, Albany, New York, USA

${ }^{3}$ Department of Physics, University of Alberta, Edmonton, Alberta, Canada

${ }^{4 a}$ Department of Physics, Ankara University, Ankara, Turkey

${ }^{4 b}$ Istanbul Aydin University, Istanbul, Turkey

${ }^{4 \mathrm{c}}$ Division of Physics, TOBB University of Economics and Technology, Ankara, Turkey

${ }^{5}$ LAPP, Université Grenoble Alpes, Université Savoie Mont Blanc, CNRS/IN2P3, Annecy, France

${ }^{6}$ High Energy Physics Division, Argonne National Laboratory, Argonne, Illinois, USA

${ }^{7}$ Department of Physics, University of Arizona, Tucson, Arizona, USA

${ }^{8}$ Department of Physics, University of Texas at Arlington, Arlington, Texas, USA

${ }^{9}$ Physics Department, National and Kapodistrian University of Athens, Athens, Greece

${ }^{10}$ Physics Department, National Technical University of Athens, Zografou, Greece

${ }^{11}$ Department of Physics, University of Texas at Austin, Austin, Texas, USA

${ }^{12 a}$ Bahcesehir University, Faculty of Engineering and Natural Sciences, Istanbul, Turkey

${ }^{12 b}$ Istanbul Bilgi University, Faculty of Engineering and Natural Sciences, Istanbul, Turkey

${ }^{12 \mathrm{C}}$ Department of Physics, Bogazici University, Istanbul, Turkey

${ }^{12 \mathrm{~d}}$ Department of Physics Engineering, Gaziantep University, Gaziantep, Turkey

${ }^{13}$ Institute of Physics, Azerbaijan Academy of Sciences, Baku, Azerbaijan

${ }^{14}$ Institut de Física d'Altes Energies (IFAE), Barcelona Institute of Science and Technology, Barcelona, Spain

${ }^{15 a}$ Institute of High Energy Physics, Chinese Academy of Sciences, Beijing, China

${ }^{15 \mathrm{~b}}$ Physics Department, Tsinghua University, Beijing, China

${ }^{15 c}$ Department of Physics, Nanjing University, Nanjing, China

${ }^{15 \mathrm{~d}}$ University of Chinese Academy of Science (UCAS), Beijing, China

${ }^{16}$ Institute of Physics, University of Belgrade, Belgrade, Serbia

${ }^{17}$ Department for Physics and Technology, University of Bergen, Bergen, Norway

${ }^{18}$ Physics Division, Lawrence Berkeley National Laboratory and University of California, Berkeley, California, USA

${ }^{19}$ Institut für Physik, Humboldt Universität zu Berlin, Berlin, Germany

${ }^{20}$ Albert Einstein Center for Fundamental Physics and Laboratory for High Energy Physics, University of Bern, Bern, Switzerland

${ }^{21}$ School of Physics and Astronomy, University of Birmingham, Birmingham, United Kingdom

${ }^{22}$ Centro de Investigaciónes, Universidad Antonio Nariño, Bogota, Colombia

${ }^{23 a}$ Dipartimento di Fisica e Astronomia, Università di Bologna, Bologna, Italy

${ }^{23 \mathrm{~b}}$ INFN Sezione di Bologna, Italy

${ }^{24}$ Physikalisches Institut, Universität Bonn, Bonn, Germany

${ }^{25}$ Department of Physics, Boston University, Boston, Massachusetts, USA

${ }^{26}$ Department of Physics, Brandeis University, Waltham, Massachusetts, USA

${ }^{27}$ Transilvania University of Brasov, Brasov, Romania

${ }^{27 \mathrm{~b}}$ Horia Hulubei National Institute of Physics and Nuclear Engineering, Bucharest, Romania

${ }^{27 c}$ Department of Physics, Alexandru Ioan Cuza University of Iasi, Iasi, Romania

${ }^{27 \mathrm{~d}}$ National Institute for Research and Development of Isotopic and Molecular Technologies,

Physics Department, Cluj-Napoca, Romania

${ }^{27 e}$ University Politehnica Bucharest, Bucharest, Romania

${ }^{27 \mathrm{f}}$ West University in Timisoara, Timisoara, Romania

${ }^{28 a}$ Faculty of Mathematics, Physics and Informatics, Comenius University, Bratislava, Slovak Republic

${ }^{28 \mathrm{~b}}$ Department of Subnuclear Physics, Institute of Experimental Physics of the Slovak Academy of Sciences,

Kosice, Slovak Republic

${ }^{29}$ Physics Department, Brookhaven National Laboratory, Upton, New York, USA

${ }^{30}$ Departamento de Física, Universidad de Buenos Aires, Buenos Aires, Argentina

${ }^{31}$ Cavendish Laboratory, University of Cambridge, Cambridge, United Kingdom

${ }^{32 a}$ Department of Physics, University of Cape Town, Cape Town, South Africa

${ }^{32 \mathrm{~b}}$ Department of Mechanical Engineering Science, University of Johannesburg, Johannesburg, South Africa 
${ }^{32 \mathrm{c}}$ School of Physics, University of the Witwatersrand, Johannesburg, South Africa

${ }^{33}$ Department of Physics, Carleton University, Ottawa, Ontario, Canada

${ }^{34 a}$ Faculté des Sciences Ain Chock, Réseau Universitaire de Physique des Hautes Energies-Université Hassan II, Casablanca, Morocco

${ }^{34 \mathrm{~b}}$ Centre National de l'Energie des Sciences Techniques Nucleaires (CNESTEN), Rabat, Morocco

${ }^{34 \mathrm{c}}$ Faculté des Sciences Semlalia, Université Cadi Ayyad, LPHEA-Marrakech, Morocco

${ }^{34 \mathrm{~d}}$ Faculté des Sciences, Université Mohamed Premier and LPTPM, Oujda, Morocco

${ }^{34 \mathrm{e}}$ Faculté des sciences, Université Mohammed V, Rabat, Morocco

${ }^{35}$ CERN, Geneva, Switzerland

${ }^{36}$ Enrico Fermi Institute, University of Chicago, Chicago, Illinois, USA

${ }^{37}$ LPC, Université Clermont Auvergne, CNRS/IN2P3, Clermont-Ferrand, France

${ }^{38}$ Nevis Laboratory, Columbia University, Irvington, New York, USA

${ }^{39}$ Niels Bohr Institute, University of Copenhagen, Copenhagen, Denmark

${ }^{40 a}$ Dipartimento di Fisica, Università della Calabria, Rende, Italy

${ }^{40 \mathrm{~b}}$ INFN Gruppo Collegato di Cosenza, Laboratori Nazionali di Frascati, Italy

${ }^{41}$ Physics Department, Southern Methodist University, Dallas, Texas, USA

${ }^{42}$ Physics Department, University of Texas at Dallas, Richardson, Texas, USA

${ }^{43 a}$ Department of Physics, Stockholm University, Sweden

${ }^{43 \mathrm{~b}}$ Oskar Klein Centre, Stockholm, Sweden

${ }^{44}$ Deutsches Elektronen-Synchrotron DESY, Hamburg and Zeuthen, Germany

${ }^{45}$ Lehrstuhl für Experimentelle Physik IV, Technische Universität Dortmund, Dortmund, Germany

${ }^{46}$ Institut für Kern- und Teilchenphysik, Technische Universität Dresden, Dresden, Germany

${ }^{47}$ Department of Physics, Duke University, Durham, North Carolina, USA

${ }^{48}$ SUPA-School of Physics and Astronomy, University of Edinburgh, Edinburgh, United Kingdom

${ }^{49}$ INFN e Laboratori Nazionali di Frascati, Frascati, Italy

${ }^{50}$ Physikalisches Institut, Albert-Ludwigs-Universität Freiburg, Freiburg, Germany

${ }^{51}$ II. Physikalisches Institut, Georg-August-Universität Göttingen, Göttingen, Germany

${ }^{52}$ Département de Physique Nucléaire et Corpusculaire, Université de Genève, Genève, Switzerland

${ }^{53 a}$ Dipartimento di Fisica, Università di Genova, Genova, Italy

${ }^{53 \mathrm{~b}}$ INFN Sezione di Genova, Italy

${ }^{54}$ II. Physikalisches Institut, Justus-Liebig-Universität Giessen, Giessen, Germany

${ }^{55}$ SUPA-School of Physics and Astronomy, University of Glasgow, Glasgow, United Kingdom

${ }^{56}$ LPSC, Université Grenoble Alpes, CNRS/IN2P3, Grenoble INP, Grenoble, France

${ }^{57}$ Laboratory for Particle Physics and Cosmology, Harvard University, Cambridge, Massachusetts, USA

${ }^{58 a}$ Department of Modern Physics and State Key Laboratory of Particle Detection and Electronics,

University of Science and Technology of China, Hefei, China

${ }^{58 \mathrm{~b}}$ Institute of Frontier and Interdisciplinary Science and Key Laboratory of Particle Physics and Particle Irradiation (MOE), Shandong University, Qingdao, China

${ }^{58 c}$ School of Physics and Astronomy, Shanghai Jiao Tong University, KLPPAC-MoE, SKLPPC, Shanghai, China

${ }^{58 \mathrm{~d}}$ Tsung-Dao Lee Institute, Shanghai, China

${ }^{59 a}$ Kirchhoff-Institut für Physik, Ruprecht-Karls-Universität Heidelberg, Heidelberg, Germany

${ }^{59 \mathrm{~b}}$ Physikalisches Institut, Ruprecht-Karls-Universität Heidelberg, Heidelberg, Germany

${ }^{60}$ Faculty of Applied Information Science, Hiroshima Institute of Technology, Hiroshima, Japan

${ }^{61 a}$ Department of Physics, Chinese University of Hong Kong, Shatin, N.T., Hong Kong, China

${ }^{61 \mathrm{~b}}$ Department of Physics, University of Hong Kong, Hong Kong, China

${ }^{61 \mathrm{c}}$ Department of Physics and Institute for Advanced Study, Hong Kong University of Science and Technology, Clear Water Bay, Kowloon, Hong Kong, China

${ }^{62}$ Department of Physics, National Tsing Hua University, Hsinchu, Taiwan

${ }^{63}$ Department of Physics, Indiana University, Bloomington, Indiana, USA

${ }^{64 \mathrm{a}}$ INFN Gruppo Collegato di Udine, Sezione di Trieste, Udine, Italy

${ }^{64 \mathrm{~b}}$ ICTP, Trieste, Italy

${ }^{64 \mathrm{c}}$ Dipartimento di Chimica, Fisica e Ambiente, Università di Udine, Udine, Italy

${ }^{65 a}$ INFN Sezione di Lecce, Italy

${ }^{65 \mathrm{~b}}$ Dipartimento di Matematica e Fisica, Università del Salento, Lecce, Italy

${ }^{66 a}$ INFN Sezione di Milano, Italy

${ }^{66 \mathrm{~b}}$ Dipartimento di Fisica, Università di Milano, Milano, Italy

${ }^{67}$ INFN Sezione di Napoli, Italy

${ }^{67 \mathrm{~b}}$ Dipartimento di Fisica, Università di Napoli, Napoli, Italy

${ }^{68 \mathrm{a}}$ INFN Sezione di Pavia, Italy 
${ }^{68 \mathrm{~b}}$ Dipartimento di Fisica, Università di Pavia, Pavia, Italy

${ }^{69}$ INFN Sezione di Pisa, Italy

${ }^{69 \mathrm{~b}}$ Dipartimento di Fisica E. Fermi, Università di Pisa, Pisa, Italy

${ }^{70 a}$ INFN Sezione di Roma, Italy

${ }^{70 \mathrm{~b}}$ Dipartimento di Fisica, Sapienza Università di Roma, Roma, Italy

${ }^{71 a}$ INFN Sezione di Roma Tor Vergata, Italy

${ }^{71 \mathrm{~b}}$ Dipartimento di Fisica, Università di Roma Tor Vergata, Roma, Italy

${ }^{72 \mathrm{a}}$ INFN Sezione di Roma Tre, Italy

${ }^{72 \mathrm{~b}}$ Dipartimento di Matematica e Fisica, Università Roma Tre, Roma, Italy

${ }^{73 a}$ INFN-TIFPA, Italy

${ }^{73 b}$ Università degli Studi di Trento, Trento, Italy

${ }^{74}$ Institut für Astro- und Teilchenphysik, Leopold-Franzens-Universität, Innsbruck, Austria

${ }^{75}$ University of Iowa, Iowa City, Iowa, USA

${ }^{76}$ Department of Physics and Astronomy, Iowa State University, Ames, Iowa, USA

${ }^{77}$ Joint Institute for Nuclear Research, Dubna, Russia

${ }^{78}$ Departamento de Engenharia Elétrica, Universidade Federal de Juiz de Fora (UFJF), Juiz de Fora, Brazil

${ }^{78 b}$ Universidade Federal do Rio De Janeiro COPPE/EE/IF, Rio de Janeiro, Brazil

${ }^{78 c}$ Universidade Federal de São João del Rei (UFSJ), São João del Rei, Brazil

${ }^{78 \mathrm{~d}}$ Instituto de Física, Universidade de São Paulo, São Paulo, Brazil

${ }^{79}$ KEK, High Energy Accelerator Research Organization, Tsukuba, Japan

${ }^{80}$ Graduate School of Science, Kobe University, Kobe, Japan

${ }^{81 \mathrm{a}}$ AGH University of Science and Technology, Faculty of Physics and Applied Computer Science, Krakow, Poland

${ }^{81 \mathrm{~b}}$ Marian Smoluchowski Institute of Physics, Jagiellonian University, Krakow, Poland

${ }^{82}$ Institute of Nuclear Physics Polish Academy of Sciences, Krakow, Poland

${ }^{83}$ Faculty of Science, Kyoto University, Kyoto, Japan

${ }^{84}$ Kyoto University of Education, Kyoto, Japan

${ }^{85}$ Research Center for Advanced Particle Physics and Department of Physics, Kyushu University, Fukuoka, Japan

${ }^{86}$ Instituto de Física La Plata, Universidad Nacional de La Plata and CONICET, La Plata, Argentina

${ }^{87}$ Physics Department, Lancaster University, Lancaster, United Kingdom

${ }^{88}$ Oliver Lodge Laboratory, University of Liverpool, Liverpool, United Kingdom

${ }^{89}$ Department of Experimental Particle Physics, Jožef Stefan Institute and Department of Physics, University of Ljubljana, Ljubljana, Slovenia

${ }^{90}$ School of Physics and Astronomy, Queen Mary University of London, London, United Kingdom

${ }^{91}$ Department of Physics, Royal Holloway University of London, Egham, United Kingdom

${ }^{92}$ Department of Physics and Astronomy, University College London, London, United Kingdom

${ }^{93}$ Louisiana Tech University, Ruston, Louisiana, USA

${ }^{94}$ Fysiska institutionen, Lunds universitet, Lund, Sweden

${ }^{95}$ Centre de Calcul de l'Institut National de Physique Nucléaire et de Physique des Particules (IN2P3), Villeurbanne, France

${ }^{96}$ Departamento de Física Teorica C-15 and CIAFF, Universidad Autónoma de Madrid, Madrid, Spain

${ }^{97}$ Institut für Physik, Universität Mainz, Mainz, Germany

${ }^{98}$ School of Physics and Astronomy, University of Manchester, Manchester, United Kingdom

${ }^{99}$ CPPM, Aix-Marseille Université, CNRS/IN2P3, Marseille, France

${ }^{100}$ Department of Physics, University of Massachusetts, Amherst, Massachusetts, USA

${ }^{101}$ Department of Physics, McGill University, Montreal, Québec, Canada

${ }^{102}$ School of Physics, University of Melbourne, Victoria, Australia

${ }^{103}$ Department of Physics, University of Michigan, Ann Arbor, Michigan, USA

${ }^{104}$ Department of Physics and Astronomy, Michigan State University, East Lansing, Michigan, USA

${ }^{105}$ B. I. Stepanov Institute of Physics, National Academy of Sciences of Belarus, Minsk, Belarus

${ }^{106}$ Research Institute for Nuclear Problems of Byelorussian State University, Minsk, Belarus

${ }^{107}$ Group of Particle Physics, University of Montreal, Montreal, Québec, Canada

${ }^{108}$ P. N. Lebedev Physical Institute of the Russian Academy of Sciences, Moscow, Russia

${ }^{109}$ Institute for Theoretical and Experimental Physics (ITEP), Moscow, Russia

${ }^{110}$ National Research Nuclear University MEPhI, Moscow, Russia

${ }^{111}$ D. V. Skobeltsyn Institute of Nuclear Physics, M. V. Lomonosov Moscow State University, Moscow, Russia

${ }^{112}$ Fakultät für Physik, Ludwig-Maximilians-Universität München, München, Germany 
${ }^{113}$ Max-Planck-Institut für Physik (Werner-Heisenberg-Institut), München, Germany

${ }^{114}$ Nagasaki Institute of Applied Science, Nagasaki, Japan

${ }^{115}$ Graduate School of Science and Kobayashi-Maskawa Institute, Nagoya University, Nagoya, Japan

${ }^{116}$ Department of Physics and Astronomy, University of New Mexico, Albuquerque, New Mexico, USA

${ }^{117}$ Institute for Mathematics, Astrophysics and Particle Physics, Radboud University Nijmegen/Nikhef, Nijmegen, Netherlands

${ }^{118}$ Nikhef National Institute for Subatomic Physics and University of Amsterdam, Amsterdam, Netherlands

${ }^{119}$ Department of Physics, Northern Illinois University, DeKalb, Illinois, USA

${ }^{120 \mathrm{a}}$ Budker Institute of Nuclear Physics, SB RAS, Novosibirsk, Russia

${ }^{120 \mathrm{~b}}$ Novosibirsk State University Novosibirsk, Russia

${ }^{121}$ Department of Physics, New York University, New York, New York, USA

${ }^{122}$ Ohio State University, Columbus, Ohio, USA

${ }^{123}$ Faculty of Science, Okayama University, Okayama, Japan

${ }^{124}$ Homer L. Dodge Department of Physics and Astronomy, University of Oklahoma, Norman, Oklahoma, USA

${ }^{125}$ Department of Physics, Oklahoma State University, Stillwater, Oklahoma, USA

${ }^{126}$ Palacký University, RCPTM, Joint Laboratory of Optics, Olomouc, Czech Republic

${ }^{127}$ Center for High Energy Physics, University of Oregon, Eugene, Oregon, USA

${ }^{128}$ LAL, Université Paris-Sud, CNRS/IN2P3, Université Paris-Saclay, Orsay, France

${ }^{129}$ Graduate School of Science, Osaka University, Osaka, Japan

${ }^{130}$ Department of Physics, University of Oslo, Oslo, Norway

${ }^{131}$ Department of Physics, Oxford University, Oxford, United Kingdom

${ }^{132}$ LPNHE, Sorbonne Université, Paris Diderot Sorbonne Paris Cité, CNRS/IN2P3, Paris, France

${ }^{133}$ Department of Physics, University of Pennsylvania, Philadelphia, Pennsylvania, USA

${ }^{134}$ Konstantinov Nuclear Physics Institute of National Research Centre "Kurchatov Institute", PNPI, St. Petersburg, Russia

${ }^{135}$ Department of Physics and Astronomy, University of Pittsburgh, Pittsburgh, Pennsylvania, USA

${ }^{136 a}$ Laboratório de Instrumentação e Física Experimental de Partículas-LIP, Portugal

${ }^{136 b}$ Departamento de Física, Faculdade de Ciências, Universidade de Lisboa, Lisboa, Portugal

${ }^{136 \mathrm{c}}$ Departamento de Física, Universidade de Coimbra, Coimbra, Portugal

${ }^{136 \mathrm{~d}}$ Centro de Física Nuclear da Universidade de Lisboa, Lisboa, Portugal

${ }^{136 e}$ Departamento de Física, Universidade do Minho, Braga, Portugal

${ }^{136 f}$ Departamento de Física Teorica y del Cosmos, Universidad de Granada, Granada (Spain), Spain

${ }^{136 g}$ Dep Física and CEFITEC of Faculdade de Ciências e Tecnologia, Universidade Nova de Lisboa, Caparica, Portugal

${ }^{137}$ Institute of Physics, Academy of Sciences of the Czech Republic, Prague, Czech Republic

${ }^{138}$ Czech Technical University in Prague, Prague, Czech Republic

${ }^{139}$ Charles University, Faculty of Mathematics and Physics, Prague, Czech Republic

${ }^{140}$ State Research Center Institute for High Energy Physics, NRC KI, Protvino, Russia

${ }^{141}$ Particle Physics Department, Rutherford Appleton Laboratory, Didcot, United Kingdom

${ }^{142}$ IRFU, CEA, Université Paris-Saclay, Gif-sur-Yvette, France

${ }^{143}$ Santa Cruz Institute for Particle Physics, University of California Santa Cruz, Santa Cruz, California, USA

${ }^{144 a}$ Departamento de Física, Pontificia Universidad Católica de Chile, Santiago, Chile

${ }^{144 \mathrm{~b}}$ Departamento de Física, Universidad Técnica Federico Santa María, Valparaíso, Chile

${ }^{145}$ Department of Physics, University of Washington, Seattle, Washington, USA

${ }^{146}$ Department of Physics and Astronomy, University of Sheffield, Sheffield, United Kingdom

${ }^{147}$ Department of Physics, Shinshu University, Nagano, Japan

${ }^{148}$ Department Physik, Universität Siegen, Siegen, Germany

${ }^{149}$ Department of Physics, Simon Fraser University, Burnaby, British Columbia, Canada

${ }^{150}$ SLAC National Accelerator Laboratory, Stanford, California, USA

${ }^{151}$ Physics Department, Royal Institute of Technology, Stockholm, Sweden

${ }^{152}$ Departments of Physics and Astronomy, Stony Brook University, Stony Brook, New York, USA

${ }^{153}$ Department of Physics and Astronomy, University of Sussex, Brighton, United Kingdom

${ }^{154}$ School of Physics, University of Sydney, Sydney, Australia

${ }^{155}$ Institute of Physics, Academia Sinica, Taipei, Taiwan

${ }^{156 a}$ E. Andronikashvili Institute of Physics, Iv. Javakhishvili Tbilisi State University, Tbilisi, Georgia

${ }^{156 \mathrm{~b}}$ High Energy Physics Institute, Tbilisi State University, Tbilisi, Georgia

${ }^{157}$ Department of Physics, Technion, Israel Institute of Technology, Haifa, Israel

${ }^{158}$ Raymond and Beverly Sackler School of Physics and Astronomy, Tel Aviv University, Tel Aviv, Israel 


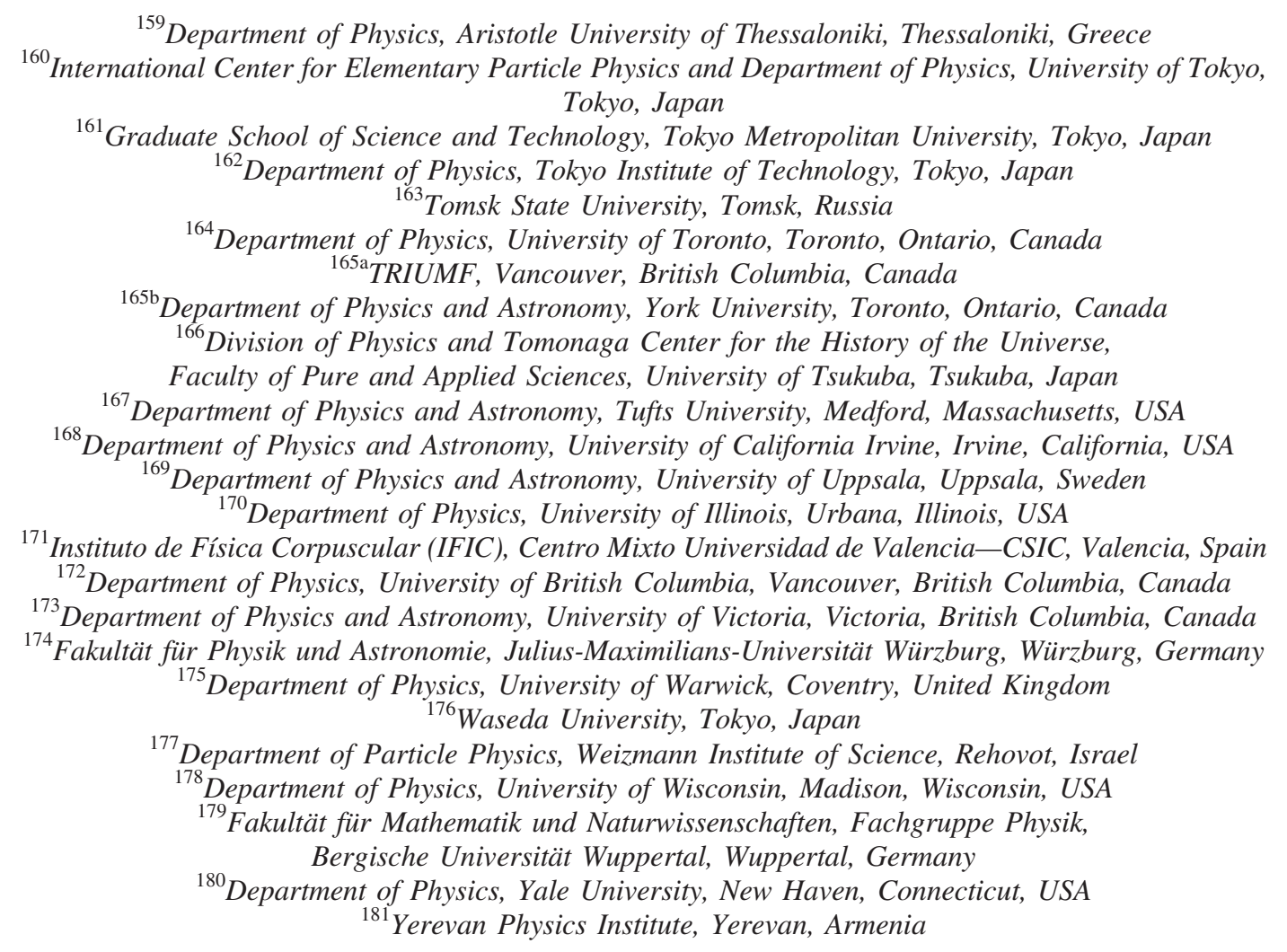

${ }^{\mathrm{a}}$ Deceased.

${ }^{\mathrm{b}}$ Also at Department of Physics, King's College London, London, United Kingdom.

${ }^{\mathrm{c}}$ Also at Istanbul University, Department of Physics, Istanbul, Turkey.

${ }^{\mathrm{d}}$ Also at Institute of Physics, Azerbaijan Academy of Sciences, Baku, Azerbaijan.

${ }^{\mathrm{e}}$ Also at TRIUMF, Vancouver, British Columbia, Canada.

${ }^{\mathrm{f}}$ Also at Department of Physics and Astronomy, University of Louisville, Louisville, Kentucky, USA.

${ }^{\mathrm{g}}$ Also at Department of Physics, California State University, Fresno, California, USA.

${ }^{\mathrm{h}}$ Also at Department of Physics, University of Fribourg, Fribourg, Switzerland.

${ }^{\mathrm{i}}$ Also at II. Physikalisches Institut, Georg-August-Universität Göttingen, Göttingen, Germany.

${ }^{\mathrm{j}}$ Also at Departament de Fisica de la Universitat Autonoma de Barcelona, Barcelona, Spain.

${ }^{\mathrm{k}}$ Also at Tomsk State University, Tomsk, and Moscow Institute of Physics and Technology State University, Dolgoprudny, Russia.

${ }^{1}$ Also at The Collaborative Innovation Center of Quantum Matter (CICQM), Beijing, China.

${ }^{\mathrm{m}}$ Also at Universita di Napoli Parthenope, Napoli, Italy.

${ }^{\mathrm{n}}$ Also at Institute of Particle Physics (IPP), Canada.

${ }^{\circ}$ Also at Dipartimento di Fisica E. Fermi, Università di Pisa, Pisa, Italy.

${ }^{\mathrm{p}}$ Also at Horia Hulubei National Institute of Physics and Nuclear Engineering, Bucharest, Romania.

${ }^{\mathrm{q}}$ Also at CPPM, Aix-Marseille Université, CNRS/IN2P3, Marseille, France.

${ }^{\mathrm{r}}$ Also at Department of Physics, St. Petersburg State Polytechnical University, St. Petersburg, Russia.

${ }^{\mathrm{s}}$ Also at Borough of Manhattan Community College, City University of New York, New York, USA.

${ }^{t}$ Also at Department of Financial and Management Engineering, University of the Aegean, Chios, Greece.

${ }^{\mathrm{u}}$ Also at Centre for High Performance Computing, CSIR Campus, Rosebank, Cape Town, South Africa.

${ }^{v}$ Also at Louisiana Tech University, Ruston, Louisiana, USA.

${ }^{\mathrm{w}}$ Also at Institucio Catalana de Recerca i Estudis Avancats, ICREA, Barcelona, Spain.

${ }^{\mathrm{x}}$ Also at Department of Physics, University of Michigan, Ann Arbor, Michigan, USA.

${ }^{y}$ Also at LAL, Université Paris-Sud, CNRS/IN2P3, Université Paris-Saclay, Orsay, France.

${ }^{\mathrm{z}}$ Also at Graduate School of Science, Osaka University, Osaka, Japan.

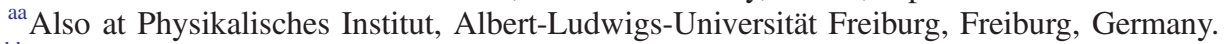

${ }^{\mathrm{bb}}$ Also at Institute for Mathematics, Astrophysics and Particle Physics, Radboud University Nijmegen/Nikhef, Nijmegen, Netherlands.

${ }^{\mathrm{cc}}$ Also at Near East University, Nicosia, North Cyprus, Mersin, Turkey.

${ }^{\mathrm{dd}}$ Also at Institute of Theoretical Physics, Ilia State University, Tbilisi, Georgia.

${ }^{\mathrm{ee}}$ Also at CERN, Geneva, Switzerland. 
${ }^{\mathrm{ff}}$ Also at Manhattan College, New York, New York, USA.

${ }^{g g}$ Also at Hellenic Open University, Patras, Greece.

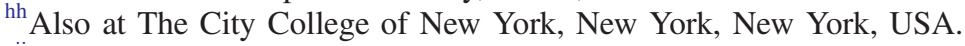

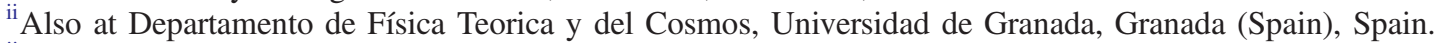

${ }^{\mathrm{jj}}$ Also at Department of Physics, California State University, Sacramento, California, USA.

${ }^{\mathrm{kk}}$ Also at Moscow Institute of Physics and Technology State University, Dolgoprudny, Russia.

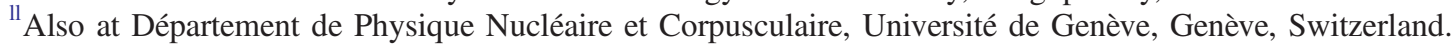

${ }^{\mathrm{mm}}$ Also at Department of Physics and Astronomy, University of Sheffield, Sheffield, United Kingdom.

${ }^{n n}$ Also at School of Physics, Sun Yat-sen University, Guangzhou, China.

${ }^{\text {oo }}$ Also at Department of Applied Physics and Astronomy, University of Sharjah, Sharjah, United Arab Emirates.

${ }^{\mathrm{pp}}$ Also at Institut für Experimentalphysik, Universität Hamburg, Hamburg, Germany.

${ }^{\mathrm{qq}}$ Also at National Research Nuclear University MEPhI, Moscow, Russia.

${ }^{\mathrm{rr}}$ Also at Institute for Particle and Nuclear Physics, Wigner Research Centre for Physics, Budapest, Hungary.

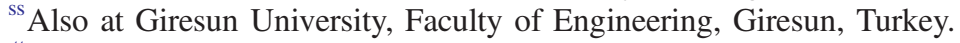

${ }^{t t}$ Also at Department of Physics, Nanjing University, Nanjing, China.

${ }^{u u}$ Also at Institute of Physics, Academia Sinica, Taipei, Taiwan.

${ }^{v v}$ Also at Department of Physics, University of Malaya, Kuala Lumpur, Malaysia. 